\title{
GEO-COORDINATED PARALLEL COORDINATES (GCPC): A CASE STUDY OF ENVIRONMENTAL DATA ANALYSIS
}

\author{
A THESIS \\ Submitted to the Faculty of GRaduate Studies and Research \\ In PARTIAL Fulfillment OF THE REQUIREMENTS \\ FOR THE DEGREE OF \\ MASTER OF SCIENCE \\ IN \\ COMPUTER SCIENCE \\ UNIVERSITY OF REGINA
}

\begin{abstract}
By
Maha Mohamed Nabil El Meseery

Regina, Saskatchewan
\end{abstract}

March 2016

Copytright (c) 2016: M. M. N. El Meseery 


\section{UNIVERSITY OF REGINA}

\section{FACULTY OF GRADUATE STUDIES AND RESEARCH SUPERVISORY AND EXAMINING COMMITTEE}

Maha Mohamed Nabil El Meseery, candidate for the degree of Master of Science in Computer Science, has presented a thesis titled, Geo-Coordinated Parallel Coordinates (GCPC): A Case Study of Environmental Data Analysis, in an oral examination held on March 21, 2016. The following committee members have found the thesis acceptable in form and content, and that the candidate demonstrated satisfactory knowledge of the subject material.

External Examiner: Dr. Joseph Piwowar, Department of Geography

Supervisor: $\quad$ Dr. Orland Hoeber, Department of Computer Science

Committee Member: Dr. Xue-Dong Yang, Department of Computer Science

Committee Member: $\quad$ Dr. Malek Mouhoub, Department of Computer Science

Chair of Defense: $\quad$ Prof. Andrew Cameron, Department of Biology 


\section{Abstract}

The large number of environmental problems faced by society in recent years has driven researchers to collect and study massive amounts of environmental data. Such environmental datasets are often high dimensional and heterogeneous in nature, with complex temporal and geospatial relations. The ability to understand and extract meaningful information from such datasets is an essential step for effective decision making. However, reasoning about the data and discovering knowledge in environmental datasets is a challenging problem due to the complexity of the data. The goal of this research is to investigate techniques to support exploration and analysis of environmental data. Such complex data could be characterized as high dimensional heterogeneous geotemporal data.

The research focused on the design, implementation, and study of approaches that facilitate the exploration and understanding of such data. A number of visualization approaches have been developed and studied to support the exploration and analysis among environmental datasets, including parallel coordinate plots, geovisualization, investigative scatterplot, and multiple coordinated views. The result of this work was the development of Geo-Coordinated Parallel Coordinates (GCPC), a geovisual analytics approach designed to support the exploration of complex environmental data. Multiple coordinated views are

used to represent the high dimensional, heterogeneous, temporal, and geospatial aspects of the data. The approach uses various interactions and analysis features to support exploring and making sense of the data. 
Field trials were conducted to validate the benefit of the approach in the analysis of environmental data with experts. Environmental analysts used the system to explore within two real datasets in their domain. The results of the evaluation were very positive in general, which provided evidence of the advantages of using the system in exploration among the complex datasets. Domain experts were able to investigate the relations between multiple heterogeneous factors while remaining aware with the geospatial aspect of the data. However, the environmental analysts saw the system as a preliminary exploration tool rather than an analytical approach. 


\section{Acknowledgments}

I would like to express my very great appreciation to Dr. Orland Hoeber for his valuable guidance and continuous support. His guidance helped me in all the time of research and writing of this thesis. I could not have imagined having a better advisor and mentor for my studies. Besides my supervisor, I would like to thank the rest of my thesis committee: Dr. Malek Mouhoub and Dr. Xue Dong Yang, for their advice and assistance in keeping the defence on schedule. I wish to acknowledge the help provided by members of the Too Big To Ignore (TBTI) project. I am particularly grateful for the assistance and feedback given throughout this research by Dr. Ratana Chuenpagdee, Dr. Rodolphe Devillers, Dr. Ellen Hines, and Dr. Rebecca Lewison. I also thank Dr. Richard Pollnac for his contribution by providing the datasets used in this work. I am gratefully thankful for the funding I received from the Social Sciences and Humanities Research Council of Canada (SSHRC) through the TBTI project. I also want to thank the Department of Computer Science for their support through sessional lecturer position and the Faculty of Graduate Studies and Research for their support through scholarships and travel grants.

My thanks also extend to all members of my research group for the stimulating discussions, for the sleepless nights we were working together before deadlines, and for all the fun we have had in the last two years. Special thanks go to my dear friend Dina Said for her support and continuous encouragement. Last but not least I thank my parents and my family for their support and encouragement throughout my study. 


\section{Dedication}

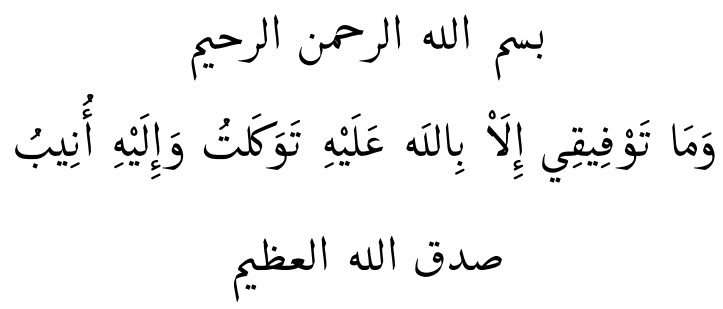

And my guidance cannot come except from Allah, in Him I trust and unto Him I repent.

This thesis is dedicated to the one who loves me the most and the people I love the most. To my mother for all the wishes and prayers she had given me. For all the time she had endured for all of us, her children. To my sisters, Kholoud for her support and Maha my sister from another mother for all laughers, love, and innocence she brought to my life. 


\section{Contents}

$\begin{array}{ll}\text { Abstract } & \text { i }\end{array}$

Acknowledgments iii

Dedication iv

$\begin{array}{ll}\text { Table of Contents } & \mathbf{v}\end{array}$

Acronyms 1

\begin{tabular}{|l|l}
\hline Chapter 1 & Introduction
\end{tabular}

1.1 Motivation . . . . . . . . . . . . . . . . . 3

1.1.1 High Dimensional Heterogeneous Geotemporal Data . . . . . . . . 4

1.1.2 Environmental Data . . . . . . . . . . . . . 5

1.2 Challenges of High Dimensional Heterogeneous Geotemporal Data Analysis 7

1.3 Adopted Approach $\ldots \ldots \ldots$. . . . . . . . . . . . . . . . 9 9

1.4 Structure of Thesis $\ldots \ldots \ldots \ldots \ldots \ldots$

\begin{tabular}{lll}
\hline Chapter 2 & Literature Review & 13
\end{tabular}

2.1 Information Visualization . . . . . . . . . . . . . . . . . . 13

$2.2 \quad$ Visual Analytics . . . . . . . . . . . . . . . . . . . . . . . . . . 19

2.3 High Dimensional Data Visualization $\ldots \ldots \ldots \ldots$. . . . . . . . 22 
2.3 .1 Dimensional Subsetting Approaches $\ldots \ldots \ldots \ldots$

2.3 .2 Dimensional Reduction Approaches . . . . . . . . . . . . . . 23

2.3 .3 Small Multiples Approaches $\ldots \ldots \ldots$. . . . . . . . . . 25

2.3 .4 Force Based Approaches . . . . . . . . . . . . . . . . . . . 27

2.3 .5 Glyph Plots Approaches . . . . . . . . . . . . . . . . . 28

$2.3 .6 \quad$ Parallel Coordinates Plot Approaches . . . . . . . . . . . . . . . . 29

2.3 .7 Hybrid Parallel Coordinates Approaches. . . . . . . . . . . . . . . 35

2.4 Geotemporal Data Visualization . . . . . . . . . . . . . 36

2.5 Multiple Coordinated Views $\ldots \ldots \ldots \ldots \ldots \ldots$

2.6 Environmental Data Visualization and Visual Analytics Approaches . . . . 42

$\begin{array}{lll}\text { Chapter } 3 & \text { Geo-Coordinated Parallel Coordinates } & 46\end{array}$

3.1 System Architecture. . . . . . . . . . . . . . . . . . . . . . 48

3.2 Geo-Coordinated Parallel Coordinates $\ldots \ldots \ldots \ldots$

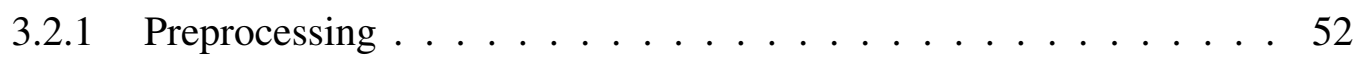

3.2 .2 Parallel Coordinates Plot . . . . . . . . . . . . . . . 53

3.2 .3 Geovisualization . . . . . . . . . . . . . . . . . . . 59

3.2 .4 Investigative Scatterplot and Correlation Analysis . . . . . . . . . 63

3.2 .5 Statistical Descriptors $\ldots \ldots \ldots \ldots \ldots$

3.2 .6 Outlier Detection . . . . . . . . . . . . . . . . . . . . . . . 69

3.2 .7 Data Inspection . . . . . . . . . . . . . . . . . 73

3.3 Exploration Scenario $\ldots \ldots \ldots \ldots$

3.4 Discussion . . . . . . . . . . . . . . . . . . . . . . . . . . 79

\begin{tabular}{lll}
\hline Chapter 4 & Field Trial Evaluations & $\mathbf{8 2}$
\end{tabular}

4.1 Introduction . . . . . . . . . . . . . . . . . . . . 82

4.2 Hypotheses $\ldots \ldots \ldots \ldots \ldots \ldots$ 
4.3 Evaluation Methodology $\ldots \ldots \ldots$. . . . . . . . . . . . . . . . 87

$4.3 .1 \quad$ Coastal Resilience Data Set $\ldots \ldots$. . . . . . . . . . . . . . . . 89

$4.3 .2 \quad$ Study Procedures $\ldots \ldots \ldots \ldots$

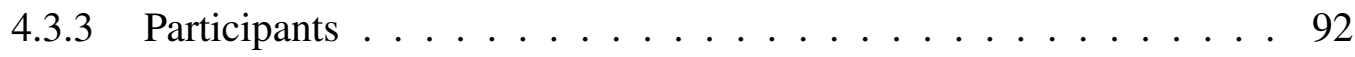

4.3 .4 Data Analysis Methods $\ldots \ldots \ldots$. . . . . . . . . . . . . 94

4.4 Results . . . . . . . . . . . . . . . . . . . . . . . . . 96

$4.4 .1 \quad$ H1: Data Exploration . . . . . . . . . . . . . . . . . 96

$4.4 .2 \quad \mathrm{H} 2:$ Knowledge Discovery $\ldots \ldots \ldots$. . . . . . . . . . . . 99

$4.4 .3 \quad$ H3: User Experience $\ldots \ldots \ldots$

4.4 .4 Focus Group Discussion $\ldots \ldots \ldots$. . . . . . . . . . . 111

4.4 .5 Observations . . . . . . . . . . . . . . . . 115

4.5 Discussion . . . . . . . . . . . . . . . . . . . . 116

\begin{tabular}{lll}
\hline Chapter 5 & Conclusions and Future Work & 121
\end{tabular}

5.1 Research Contributions . . . . . . . . . . . . . . . . . . . 121

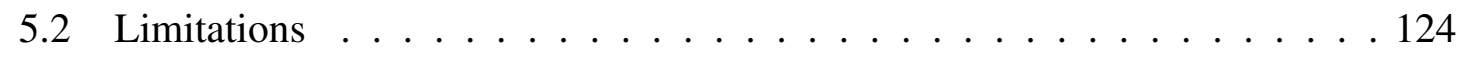

5.3 Future Work . . . . . . . . . . . . . . . . . . . 126

\begin{tabular}{ll}
\hline References & 128
\end{tabular}

\begin{tabular}{|lll}
\hline Appendix A & Research Ethics Board Approvals & 146
\end{tabular}

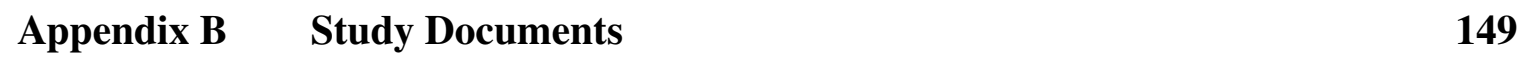




\section{List of Tables}

4.1 Demographic of participants in the field trials $\ldots \ldots \ldots$. . . . . 93

4.2 Detailed information about each measure used in the evaluation. . . . . . . 95

4.3 Summary of the evaluation results $\ldots \ldots \ldots \ldots \ldots \ldots$ 


\section{List of Figures}

$2.1 \quad$ Bertin's model of visual properties $\ldots \ldots \ldots$. . . . . . . . . . 16

2.2 Visual analytics process $\ldots \ldots \ldots \ldots$. . . . . . . . . . 20

2.3 The sense making loop . . . . . . . . . . . . . . . . 21

2.4 Multidimensional scaling example . . . . . . . . . . . . . . . 24

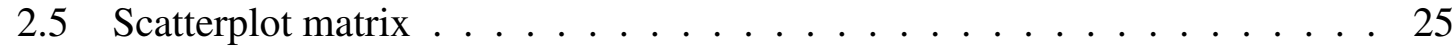

2.6 General plot matrix $\ldots \ldots \ldots \ldots \ldots$. . . . . . . . . . . 26

$2.7 \quad$ Example of glyph plot of high dimensional data $\ldots \ldots \ldots$. . . . . . . 28

2.8 Mapping a point from 2D space to parallel coordinates . . . . . . . . . . . 29

2.9 A two 6D points in parallel coordinates $\ldots \ldots \ldots$. . . . . . . . 30

2.10 Scatterplot versus parallel coordinates . . . . . . . . . . . . . 31

2.11 Densisty based parallel coordinates . . . . . . . . . . . . . . . 32

2.12 Visual cluster of parallel coordinates $\ldots \ldots \ldots$. . . . . . . . . . 34

2.13 Parallel sets . . . . . . . . . . . . . . . . . 35

2.14 Overlaying temporal data on a map . . . . . . . . . . . . . 38

2.15 Example of the space-time cube . . . . . . . . . . . . . 39

2.16 Example of small multiples geovisualization . . . . . . . . . . . . . . 40

$3.1 \quad$ Architectural diagram of $\mathrm{GCPC} \quad \ldots \ldots \ldots$

3.2 Overview of $\mathrm{GCPC} \ldots \ldots \ldots \ldots$. . . . . . . . . . . . . . 51

3.3 Parallel coordinates plot . . . . . . . . . . . . . . . 54 
3.4 Colour encoding in GCPC $\ldots \ldots \ldots \ldots \ldots$

3.5 Zooming in parallel coordinates $\ldots \ldots \ldots \ldots \ldots$

3.6 Example of the dot map and the hexbinning views . . . . . . . . . . 60

3.7 The freehand geospatial filter $\ldots \ldots \ldots \ldots \ldots \ldots$

3.8 Example of using the investigative scatterplot $\ldots \ldots \ldots \ldots$

3.9 Example of a Tukey box plot $\ldots \ldots \ldots \ldots \ldots$

3.10 Tukey box plot for multiple dimensions $\ldots \ldots \ldots$. . . . . . . . 68

3.11 Example of histogram plot for multiple dimensions $\ldots \ldots \ldots$. . . . 68

3.12 Angle based outlier detection algorithm $\ldots \ldots \ldots \ldots \ldots 71$

3.13 Inspection window $\ldots \ldots \ldots \ldots \ldots \ldots \ldots \ldots$

3.14 Exploration scenario: overview and zoom $\ldots \ldots \ldots \ldots$

3.15 Exploration scenario: furthur analysis $\ldots \ldots \ldots \ldots \ldots 77$

3.16 Exploration scenario: exploring using the geofilter. . . . . . . . . 78

4.1 Perceived usefulness of GPCP in data exploration . . . . . . . . . . . 97

4.2 Perceived usefulness of the different features in data exploration . . . . . 98

4.3 Perceived usefulness of GPCP in detailed data analysis and knowledge discovery . . . . . . . . . . . . . . . . . . . . . 100

4.4 Perceived usefulness of the different features in detailed data analysis and knowledge discovery . . . . . . . . . . . . . . . . . . 101

4.5 Job relevance of GCPC $\ldots \ldots \ldots \ldots \ldots$

4.6 Perceived usefulness of GCPC $\ldots \ldots \ldots$

4.7 Job relevance of the different features $\ldots \ldots \ldots$. . . . . . . . . . . 104

4.8 Perceived usefulness of the different features $\ldots \ldots \ldots$. . . . . . . 105

4.9 Perceived ease of use for GCPC $\ldots \ldots \ldots \ldots$

4.10 Perceived ease of use of the different features $\ldots \ldots \ldots$. . . . . 108

4.11 Output quality for GCPC $\ldots \ldots \ldots \ldots$ 
4.12 Output quality of the different features . . . . . . . . . . . . . . . 110 


\section{Acronyms}

ABOD Angle based outlier detection

DPSIR Drivers, Pressures, States, Impact, and Responses

GCPC Geo-Coordinated Parallel Coordinates

GIS Geographic Information System

MCV Multiple coordinated views

MDS Multidimensional Scaling

MPA Marine Protected Areas

PCA Principle Component Analysis

TAM Technology Acceptance Model

TAM2 Extention of the Technology Acceptance Model

VA Visual Analytics 


\section{Chapter 1}

\section{Introduction}

Data is tremendously valuable when we are able to understand it and extract knowledge from it. The ability to understand and discover knowledge from raw data is an essential step for effective decision-making in any field. To gain insights and discover new knowledge from the data, we need to synthesize information from multiple data sources, browse and explore among the data, and identify meaningful and relevant information from within the data. With the massive amount of data gathered in recent years, the process of analyzing and understanding the data is increasingly becoming a challenging problem [75].

The volume of the data is not the only problem; the high dimensionality and the heterogeneousness of the collected data contribute to the complexity of the problem [71]. Additionally, with recent advancements in sensor, mobile, and GPS technologies, temporal and spatial components are easily associated with data. The need to derive insights from this massive data is driving researchers to design, develop, and study new tools and techniques to support understanding and reasoning within the data [10, 104].

The purpose of this work is to develop a method to support the analysis and understanding of complex patterns and trends within high dimensional heterogeneous geotemporal data. This Thesis presents Geo-Coordinated Parallel Coordinates (GCPC), an approach that integrates multiple visualizations and intuitive interactions to support exploration and 
reasoning about the data [43]. This Chapter presents an introduction for the Thesis and explains the motivation for the research, the challenges of high dimensional heterogeneous geotemporal data visualization, and a summary of the approach.

\subsection{Motivation}

With the current advances in data gathering and storage technology, we are generating data faster than our ability to use it [75]. In most fields and industries, the amount of data produced is adding to the complexity of the decision making process [11, 25]. Analysts, researchers, and decision makers' ability to generate solutions and solve problems is highly dependent on their capacity to understand the collected data [11]. Analyzing and understanding such data requires synthesizing data from multiple sources, exploring the data in detail, identifying the patterns within, and making sense of the large volume of data [75, 116]. This process of discovering knowledge and extracting meaningful information from the data plays a critical role in the decision making process.

While the volume of the available data contributes to the problem, it is not the only dilemma. With advances in sensor technologies and integration of multiple sources, collected data has increased in both resolution and dimensionality [5]. The existence of high dimensional data (i.e., $N$ dimensions, where $N$ is more than the dimensions clearly visualized on a screen display $\backsim 4$ ) has become ubiquitous to a wide range of domains such as financial analysis, bioinformatics, and astronomy [40, 54, 94]. Analytical techniques used to analyze and extract knowledge from a small set of dimensions does not scale well with higher dimensions. Researchers are faced with an increase of complexity when dealing with higher dimensional space. This increase in the number of dimensions complicates the extraction of relevant and meaningful information from the data. In light of this increase of high dimensional data, there is an increasing interest in tools that support analyzing and 
understanding such data [40].

Additionally, synthesizing various data sources often generate heterogeneous data with multiple different data types. These date types could incorporate different factors such as: textual, qualitative, quantitative, and ordinal data. Depending on the type and number of these factors, the process of analyzing and understanding the data changes tremendously [45]. Due to the diversity of the factors, the process of making sense of the data and the interrelations between these factors is a challenging problem. Although some research has focused on tools to support the analysis of such data, there is a need to investigate methods and techniques to address this problem [30, 57, 85, 117].

\subsubsection{High Dimensional Heterogeneous Geotemporal Data}

Geospatial data represents information that describes a particular geographical location. Recently, geospatial data has become an important component in a wide range of domains [19, 44, 86, 117, 132]. Fields such as environmental analysis, environmental planning, and crisis management have a significant geographical and spatial aspect to the domain [19, 117]. Additionally, the latest advances in technologies have increased the access to personal geospatial data. This growth and availability of geospatial data are ex-

ploited in a wide range of scientific and industrial applications [9, 85, 86]. It is not only the location information that is essential for such applications; in many cases the geospatial data also have a vital temporal aspect.

With the increased integration of geospatial data in a wide range of applications, the need to support such data has increased in the past few years. Adding temporal and geospatial aspects of the data increases the complexity of understanding it. Geotemporal databases often hold numerous diverse attributes associated with a particular spatial location. For example, spatial data stored explains the geographical location of a specific village, non-spatial data provides details about the population, activities of the villagers, 
and other statistical information at specific time period [104]. With the diversity of the spatial and non-spatial data in geotemporal datasets, reasoning and understanding these attributes without losing awareness of the geotemporal data associated with it is challenging.

With the growing complexity of geotemporal datasets, there is an increasing need for tools to explore and make sense of such complex and diverse data. Although extensive research has been carried out on the analysis of high dimensional data and the analysis of geotemporal data separately [21, 124], research on understanding high dimensional data with geotemporal aspects is still limited [85].

\subsubsection{Environmental Data}

In the history of human development, the last 50 years have the fastest and the largest growth ever. This rapid human development is straining the Earth's resources, resulting in a negative impact on our environment [14]. The continuous and substantial demand we are placing on the environment is affecting the natural equilibrium of ecosystems. Ecosystems are communities of living organisms that interact with and affect the surrounding environment. This change to our environment and ecosystems has caused numerous environmental problems and the loss of biodiversity [14]. Environmental researchers are attempting to mitigate these problems, while meeting the demands for necessary services provided by ecosystems.

To address these environmental problems, researchers need to study how changes in ecosystems and biodiversity are influenced by humans, and how humans are affected by changes in ecosystems. Analyzing and understanding the ecosystem status is a fundamental aspect to improve ecosystem management techniques [82, 14, 113]. Developing environmental management and analysis tools is a key aspect to support environmental decision making processes [74].

However, these environments and ecosystems are dynamic systems that are undergoing 
continuous change and modification due to natural and human causes [95]. There are multiple biological, ecological, economic, and environmental factors that influence ecosystems. These influences appear in the environment at different spatial and temporal scales. Understanding the significance of these factors plays a vital role in addressing environmental problems. However, there are multiple relations and connections between these different factors, which impacts the environment differently. The complexity of multiple factors and their influences make ecosystems complex systems to understand and manage.

While the relations between the diverse factors is complex, geospatial and temporal aspects of the environmental data further complicate it. Recent satellite imaging and data collection technology allow for acquiring data that is frequent and of high resolution [19, 78]. This high volume, multiple resolutions, and scale of geotemporal data contribute to the complexity of environmental data.

In many cases, environmental data can be characterized as high dimensional heterogeneous geotemporal data. Understanding the different characteristics of the data without loosing awareness of the geotemporal aspect is a challenging problem. The multiple sources and datasets, the dimensionality of the data, and the heterogeneous factors contribute to the problem. To gain knowledge from this complex data, researchers need to explore and make sense of the large volume and diverse data with respect to the geospatial and temporal aspects. 


\subsection{Challenges of High Dimensional Heterogeneous Geotemporal Data Analysis}

With an increasing need for techniques to support analyzing and understanding of complex data in recent years, information visualization has been commonly utilized as a mechanism for data exploration and analysis [126]. Visualizing data makes it clear and effortless to interpret it at a glance, which supports gaining knowledge and understanding large amounts of data in an efficient way. Visual representation of the data facilitates identifying relevant information and detecting patterns and trends in the data.

But to effectively understand visualizations, data should be correctly and effectively encoded to graphical representations. Moreover, understanding data requires more than just visualizing them; the users have to able to explore and interact with the data, and manipulate the view to gain meaningful insights about the data. As the volume and the complexity of the data increases, visualizations must be designed with careful consideration of appropriate visual representation, encoding, and interaction techniques to support analysis and understanding of the data [127, 126].

Even though information visualization supports understanding of the of data, creating visual representations of high dimensional data is a challenging problem by itself. The main dilemma is how to represent high dimensional data in $2 \mathrm{D}$ display space without adding extra visual clutter. Several approaches have been introduced to visually represent high dimensional data, but only a few of them are scalable [5, 18, 33, 55]. Additionally, challenges include identifying patterns with large a number of dimensions, and following trends across multiple dimensions.

For simple geospatial data, various visualizations have been introduced to display geospatial data, such as: dot maps, choropleth maps, cartograms, network maps, and surface maps [104, 126]. Each of these visualizations provides the users with different viewpoints 
of the data based on the type of data and the geographical features. To understand such data, users are empowered to see the geographical distribution of the data, identify patterns and trends, and compare the different information and locations [104].

With high dimensional data, the complexity of visualizing geotemporal data increases tremendously. When the number of the dimensions increases, visualizing the data over a map becomes a difficult issue that needs consideration. There are a limited number of graphical properties that can be used in a visualization before it becomes hard to understand. This limited visual properties and the limited display space make it hard to show more than a handful of dimensions. Selecting dimensions to visualize means choosing which dimension holds relevant information beforehand. Supporting user exploration of geotemporal data is another problem. Traditional Geographic Information Systems (GIS) approaches overlay multiple layers on the map to visualize the different dimensions in the data [105, 113, 130]. Detecting and analyzing of patterns in multiple dimensions requires merging and manipulating these layers. With a high number of dimensions, this process is cognitively taxing.

The diversity and heterogeneity of the data is another complication. Supporting visual exploration and analysis of data with various types requires multiple viewpoints and visual encodings of the data. In order to facilitate reasoning, the different types of data should be visualized appropriately [104]. For example, numerical information like population over the map should be visualized differently than visualizing categorical information like industries in the area.

Additionally, adding a temporal aspect to the geospatial data introduces multiple complexities on how to display the change of time over the map. Due to the various characteristics of both the temporal and geospatial data, analyzing geotemporal data is a complex task. The multiple types and scales of geotemporal data makes it difficult to identify and analyze the patterns and trends in the data over both space and time [13]. Additionally, 
these numerous characteristics introduce multiple relations and dependencies within the data [13, 132].

This Thesis addresses challenges associated with the analysis of high dimensional heterogeneous geotemporal data. The main challenges addressed are: 1) the exploration and analysis of high dimensional data with a mix of qualitative and quantitative data; 2) the exploration and analysis of geotemporal data with multiple non-spatial attributes; and 3) the exploration and analysis of environmental data as an example of such data.

\subsection{Adopted Approach}

The main aim of this research is to investigate, design, develop, and study a visual and interactive approach to support the analysis and understanding of high dimensional heterogeneous geotemporal data. A fundamental objective of this research is to support the exploration of multiple factors within the complex heterogeneous data while remaining aware of the geotemporal aspect of the data. Allowing users to recognize and identify patterns and trends within such data permits users to affirm known information and discover new knowledge. Motivated by challenges of analyzing environmental data, the study will focus on such data as an example of high dimensional heterogeneous geotemporal data. Providing an effective visual approach to support exploration within environmental data could enhance current environmental decision making.

Visual Analytics (VA) is an emerging approach that is increasingly being employed to support exploratory analysis of data [13, 75]. By combining information visualization, data processing, data mining, and interactive interfaces, analysts are able to explore, analyze,

reason, and make sense of highly complex data [75]. Merging multiple visual and interactive representations help analysts to generate hypotheses, identify new lines of enquiry, 
understand patterns, and derive new insight from what is being shown. With these advantages, decision support systems in various fields are commonly employing visual analytics approaches [8, 78, 112].

Adding and involving the user in knowledge and understanding processes plays a vital role in visual analytics. Intuitive interactions with the system provides users with means to explore the data, locate information, confirm hypotheses, and gain new insights about the data. Acquiring new knowledge becomes a continuous loop of interaction between the user, the visualization system, and the data.

In this research, a visual analytics approach is employed to support exploring and analyzing various patterns and trends within the complex data. The research attempts to design a system that could integrate various techniques to analyze high dimensional heterogeneous geotemporal data. A geovisual analytics system called Geo-Coordinated Parallel Coordinates (GCPC) is presented to support the analysis and reasoning about such data.

GCPC uses multiple coordinated views to show, examine, and explore high dimensional heterogeneous geotemporal data. The core of the system is comprised of two tightly coupled coordinated views: a parallel coordinates plot and a geovisualization. A parallel coordinates plot is a visualization method that represents a large number of dimensions using parallel axes [69]. The parallel coordinates are used to visualize the different types of data such as: quantitative, qualitative, ordinal, and temporal data. As these dimensions are represented in the parallel coordinates using parallel axes, the approach is very flexible and scalable with respect to type and the dimensionality of the data. The geovisualization shows the geographical distribution of the data to allow for exploration within the relations of the multiple factors and the geospatial aspects of the data. The two main views allow the system to represent the high dimensional, heterogeneous, temporal, and geospatial aspects of the data simultaneously. Additionally, a scatter plot view allows analysts to interactively investigate direct correlations between multiple factors. 
To support exploration among the complex data, these visual components are linked in a type of multiple coordinated views using various interactions: filtering, zooming, and highlighting the data. To allow a deeper insight into the complex high dimensional data, micro-visualizations have been added to the parallel coordinates to represent the statistical properties of the data. Additionally, the system employs a high dimensional outlier detection to help user identify points that are different than the general distribution of a dataset.

Geo-Coordinated Parallel Coordinates is designed to enable the interactive analysis activities described in Keim's visual analytics mantra: "analyze first; show the important; zoom, filter, and analyze further; details on demand" [75]. Following this mantra, the first step is preprocessing to reformat and compute the initial distributions of the data. Next, the system shows the initial view displaying the main aspects of the data. Multiple coordinated views allow the user to investigate the data using zoom, filters, and other interactions across the different visual representations. Additional analysis tools are provided to explore direct correlations between factors, analyze outliers, and examine details about the statistical information related to the data. A detail window and table view provide the user with raw data and detail information on demand.

\subsection{Structure of Thesis}

This Thesis is composed of five chapters. Chapter 1 outlines the motivation and the main problem under study. A literature review of the main topics relevant to this work is presented in Chapter 2. The details of the GCPC system are presented in Chapter 3 , explaining the design goals and the system high level architecture, the details of each component, and the implementation specifics. Chapter 4 presents and discusses the evaluation 
methods and the details of the field trials, including the analysis of the result of the interviews and focus group discussions during the field trials. Chapter 5 concludes the Thesis and discusses the contribution of research, the limitations, and future work. 


\section{Chapter 2}

\section{Literature Review}

The use of visual analytics to support exploration, analysis, and knowledge discovery within high dimensional geotemporal data is an active research domain with applications in many fields [41, 70]. This Chapter provides a review of literature on the main topics that are relevant to this work. Section 2.1 introduces information visualization highlighting its main theories and principles. Visual analytics concepts are explained in Section 2.2. High dimensional data visualization challenges and approaches are summarized in Section 2.3. Section 2.4 explains recent approaches in geotemporal data visualization. Multiple coordinated views are discussed in Section 2.5. Finally, environmental data analytics and recent visual approaches for environmental data are reviewed in Section 2.6.

\subsection{Information Visualization}

Information visualization is defined as the use of graphical representations to present and communicate abstract data [126]. Visualizing data provides mechanisms to transform raw data into an intuitive visual format, which is easily interpreted by humans. It utilizes the power of human perception system that naturally supports the perception and interpretation

of visual representations [127]. A single graph can hold a wealth of information that can be interpreted by the humans in a short time compared to other format such as text and 
tables. This is because our perceptual system can process visual inputs in parallel, which allows for such quick interpretation of information [126]. This efficiency of information visualization supports users in gaining knowledge and understanding of large amounts of data [122]. Moreover, information visualization provides a mechanism to share information and communicate findings quickly [126].

Every visualization starts by transforming the data to graphical objects, rendering these objects to a view on a display medium, and finally a user observing the rendered visualization [126]. In each of these steps, several visualization theories and principles are employed to design visualizations that are correctly and effectively understood by humans. Designers need to pay careful attention to the type of data, what is best method to visually represent each type of data, and how humans perceive and interpret visual representations.

To graphically represent data, we need to map data values to graphical objects and properties of these objects. A number of visual features could be used to map the different data values, including position, length, size, colour, orientation, and others. Selecting a specific graphical property to encode data values depends on the type of the data and the type of users' task. Data types could be categorized into: numerical data that could be discrete or continuous (e.g., population); ordinal data that covey some order or ranking information (e.g., rating of a service or a product); categorical data with no specific order (e.g., fish species); and arbitrary data (e.g., free-form textual information) [126]. Some visual properties support better understanding of numerical data, while other properties are better suited to distinguish between different categorical information. For example, length can easily be utilized to rank numerical or ordinal data values meanwhile shape and texture are effective for distinguishing between categorical data [127].

The effectiveness of a visualization is impacted by what the users want to extract from the view and how the visualization supports their specific goals. Users' goals could be: to associate and identify similar data patterns; to rank and order data values; to detect 
anomalies in the data; or to recognize different trends in the data. Different visual properties can be used to provide better support for these goals. For example, encoding data to colour can facilitate the detection of similar data points as users will be able identify graphical objects with the same colour easily if there are not too many other colours used in the display [127].

Several models have been proposed to guide designers on how to select the best visual features based on the type of the data or/and the goals of the user [126]. Figure 2.1] shows the model developed by Bertin, which relates the visual property based on the task the user is to accomplish [16]. The model classifies users' goals to four categories: association, selection, order, and quantity. Different visual properties are illustrated to show which properties should be used for each task. For example, the model illustrates that colour is best used for associations and selections of objects, while size is best used for selection, ordering of the data, and quantifying values (Figure 2.1.

As such, the effectiveness of the visualization depends on how the data is encoded and presented. Even a small change of what graphical properties are used to encode the data can change how the user understands the data [126]. Therefore, careful attention should be taken when visually encoding the data so as not to introduce patterns that do not exist in the data. In other words, visual similarity in the presentation must correspond to a similarity in the data, and any order in the visualization must convey an order in the data [118].

Understanding how human perception works is essential to design visualization that are readable and understandable. The human perception system has two types of processing: pre-attentive processing, which is parallel, fast (i.e., less than $250 \mathrm{~ms}$ [126]), and uncontrolled; or attentive processing, which is slower, controlled, and more focused processing that occurs after pre-attentive processing [126]. Pre-attentive processing allows us to quickly identify and perceive a wide range of visual features. Among the pre-attentively 


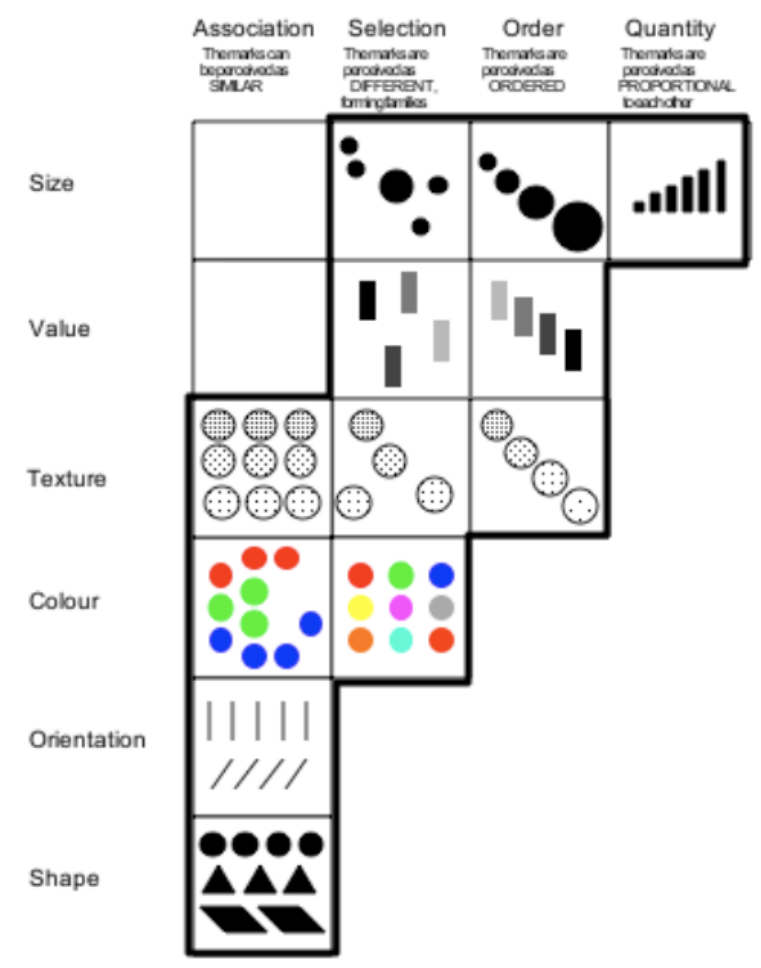

Figure 2.1: The model presented by Bertin [16] that shows different tasks and the visual properties suited for each task.

processed features are colour, size, length, orientations, and other features. Using preattentive processing we could easily identify an object if it is sufficiently different than its surroundings in one of these visual features. An example of pre-attentive processing is spotting a red object in a collection of objects that are green.

When the visual representation is more complex, more attention and cognitive effort is required to process these representations, which is why the human perception system uses attentive processing [126]. Searching for a red circle in a graph full of red squares and blue circles is an example of such processing. The red circle will not be distinctive from the surroundings elements to be detectable pre-attentively. A viewer will have to scan and remember the objects to find and identify this specific target from its surroundings [127].

Colour is a fundamental factor to consider when choosing how to encode data. Colour could be used to clarify and enhance the visualization or obscure and confuse users if 
used excessively [118, 119]. Even though colour is an important visual feature that can easily be distinguish by pre-attentive processing, there are a multiple things to consider when selecting a colour scale or range [118]. Depending on the type of data, selecting the appropriate colour scale to support users' understanding of the data is different. Categorical data should be encoded using colours that are perceptually distinct from one another, but ordered data should be encoded in colour range that is perceptually ordered.

The opponent process theory of colour [61] is a theory that allowed us to understand how we perceive colour. The theory divides colours into pairs of opposing chromatic channels: yellow-blue, red-green, and black-white [61]. Based on this theory, humans can easily distinguish colours from opposite chromatic channels. For example, a yellow object is easily identified in large group of blue objects. When representing distinct values, colours should be selected from opposing chromatic channels rather than any type of arbitrary selection. On the other hand, a monotonic variation in colour along one of these channel can be used to produce a perceptually orderly scale [93].

The Gestalt Principles [77] are useful principles that explain how we perceive patterns, foregrounds, and backgrounds. These principles demonstrate that we intuitively infer the existence of relationships between graphical objects through proximity, similarity, connectivity, and other visual organizations. These principles provide guidelines on how to generate visualizations that interpreted correctly [127]. Even thought all the Gestalt Principles are important, the most relevant principles for this work are the principle of proximity and similarity.

The proximity principle states that objects close to each are perceived as forming a group [77]. This principle also implies that when visualizing objects near each other, individuals will perceive the group as whole (i.e., as one object). Another important principle is the similarity principle, which suggests that visual objects that are similar are recognized as a group [77]. The visual similarity could exist in multiple ways such as shape, colour, 
or texture. Visualizing objects with similar visual properties will allow the observer to perceive them as part of a group and infer relation between these objects.

Supporting user interaction is essential for effective visualization. Information visualization defines interaction as the transformation of what and how the data is displayed [126]. A small change in what is displayed on the screen gives users different views and insight of the data [103, 104]. These interactions and changes allow users to have a dialogue with the data [75], which supports them in gaining more insights and understanding of the data. To support users in making sense of the data, any effective visualization should support multiple interactions. There are many interaction techniques that transform, manipulate, encode, and reconfigure the data differently, allowing user to search the data, locate patterns, and identify known or new information to make sense of the data in multiple ways [104].

Some common interactions used in visualization system include: navigation, selection, filtering, connecting, and encoding [126]. Selecting and filtering are examples of techniques used to select different subsets of the data. To select or filter data points, visualizations commonly allow brushing operations. Brushing is a term used to indicated moving the mouse over the visualization to select a subset of the data [104]. Panning and zooming are methods used to navigate through the presented information.

While navigation and filtering allow users to focus on a subset of the presented data, connection and encoding interactions provide means to examine different aspects and relationships within the data. Encoding interactions permit users to change the visual encoding of the displayed data, providing them with deeper insights exploring the different views of the same data [126]. An example of such coordination is the interaction to control the colour used to map the data on the views. Another interaction is connecting between multiple views, which allows users to identify relations between the different aspects of the data [126]. 


\subsection{Visual Analytics}

Visual Analytics (VA) is an emerging approach that is increasingly being employed to support exploratory analysis of data [13, 75]. Visual analytics aims to facilitate reasoning and understanding of massive or complex datasets by merging interactive visualizations with data transformations and analytical reasoning techniques [116]. Merging multiple visual and interactive representations helps analysts to generate hypotheses, identify new lines of enquiry, understand patterns, and derive new insights from what is being shown [75]. With these advantages, decision support systems in various fields are increasingly employing visual analytics approaches [8, 78, 112].

The quality and type of data representation used in a visualization directly impacts our understanding of the data. A key element of visual analytics is the utilization of data representation, data transformation, and data mining techniques. To improve the analytical reasoning process, visual analytics employs different techniques depending on the analytical task. Data transformation methods allow visual analytics to synthesize multiple data sources, aggregate large and complex data collections, and convert raw data into more useful forms [116]. For example, aggregation is widely used to summarize and reduce the size of data handled by the visual interface [39, 65, 90].

While data transformation algorithms convert data to a more a useful form, data mining algorithms use various techniques to extract new knowledge from the data. An example of data mining used in visual analytics systems is sentiment analysis and classification. The sentiment classes of text can be extracted from raw data. The system then visualizes these sentiment classes instead of the actual raw texts [64].

Other data mining techniques commonly used in visual analytics include: clustering, classification, and anomaly detection. Clustering algorithms are used in various visual analytics systems to group the data, and facilitate pattern and trends detection [57, 115]. 
Similar to clustering, classification algorithms are employed to label data into different categories. However, classification algorithms are usually used when the classes are known beforehand (e.g., classification of sentiments [64]). Anomaly detection algorithms are utilized in various systems to support users in finding interesting patterns in the data [90, 131].

A tight integration between the user interaction, data processing, and the visual representation is a fundamental concept in visual analytics. Figure 2.2 shows this continuous loop of automated analysis and visual exploration, which drives the visual analytics process [76]. After the first step of data transformation, interacting with the visualizations and modelling the data is controlled by the user to gain new insights about the data [75].

This visual analytics process supports the generic knowledge discovery process in the making sense loop explained by van Wikj [122]. Figure 2.3 illustrates this sense making loop for knowledge discovery in the context of the visual analytics process [122, 75]. The process starts by using automated analysis algorithms to perform the preliminary processing of the data. With the output of the initial analysis of the data, the next step is displaying the data in the visualization. These visual representations should provide users with new

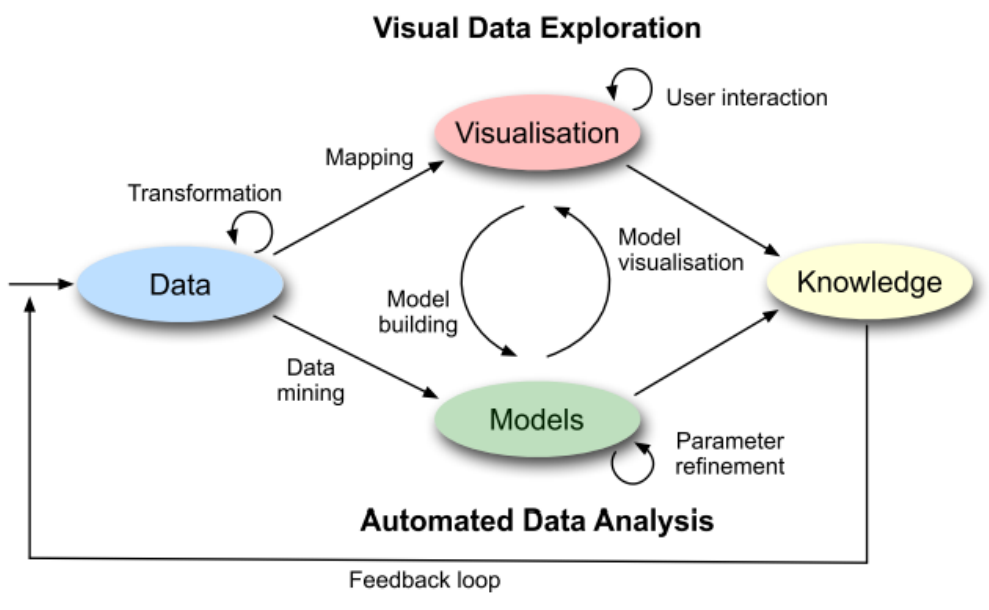

Figure 2.2: Visual analytics process [76]. 


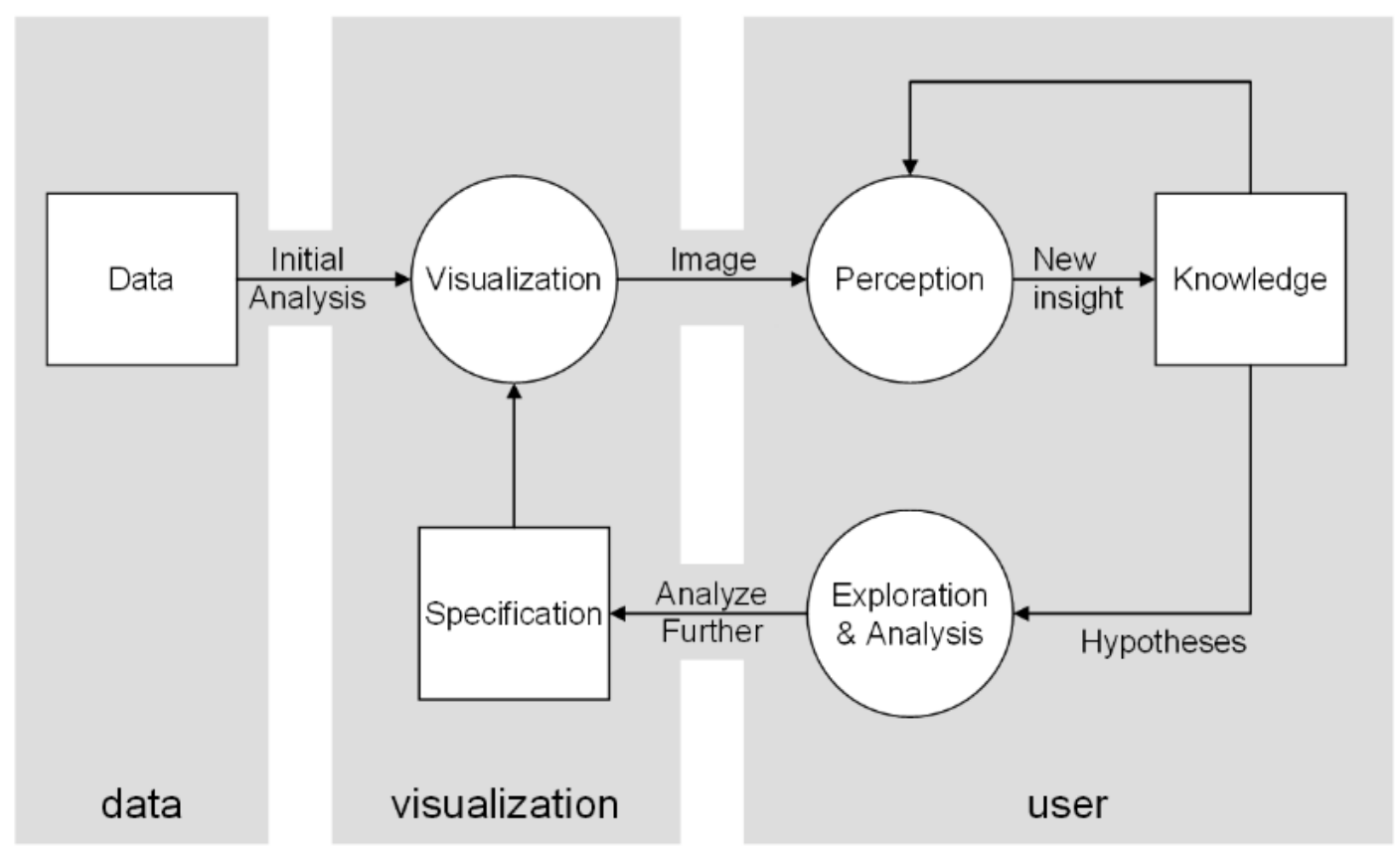

Figure 2.3: Sense making loop between the data, the visualization, and the user [122].

insight or knowledge of the data. The knowledge users gain should confirm known information or allow them to create new hypotheses about the data. To pursue their hypotheses, users should be able to and interact with the data by further exploring and analyzing the data. Gaining new knowledge is the result of this iterative process of analysis, representation, interaction, and further analysis.

To support this sense making loop, the design and implementation of visual analytics systems must allow users to go through the visual analytics process. Keim summarizes these activities in the visual analytics mantra: "analyze first; show the important; zoom, filter, and analyze further; details on demand" [75]. The mantra emphasizes the role of first analyzing the raw data before the visualization step. The next step is rendering the analyzed data and showing the most important features of the data. After that, the system should allow users to interact with the data through zooming and filtering. To support reasoning about the different subsets and aspects of the data, users should be able to interact, explore, and analyze the data. This further analysis can either be user controlled or using automated 
analysis algorithms. Finally, to allow users to verify their findings within the raw data, details of the data should be provided on demand.

\subsection{High Dimensional Data Visualization}

Data visualizations should be designed to make a clear and effective graphical representation of the data. With a small number of dimensions, the choice of how to encode the data to graphical objects is easier than with a large number of dimensions. To represent two dimensional data, designers can use a simple scatterplot and encode the data into positions relative to the $\mathrm{x}$-axis and $\mathrm{y}$-axis of the plot [126]. Additional dimensions could be encoded to shape and colour of the points. However, there is a limited number of visual properties that could be used to encode the data before it looses the clarity needed for effective visualizations. As the dimension of the data increases, the ability to map the higher dimensions to 2D display space on screen decreases. Visual clutter and complex graphical representations are a result of too many dimensions to display [118]. The problem of visualizing higher dimensions without loosing clarity to support exploration and analysis of the data is an ongoing dilemma.

Visualizing the data on the screen is not the only problem when dealing with high dimensional data. Users have to be able to explore the different dimensions, identify interesting patterns across multiple dimensions, and extract meaningful information from the data. While a multitude of approaches have been developed over the years to visualize high dimensional data, each has its limitations [55, 126]. With higher dimensions than exist on the screen, researchers have tried to handle such problems using techniques such as: dimensional subsetting, dimensional reduction, small multiples plots, force based plots, glyph plots, and parallel coordinates [55, 126]. 


\subsubsection{Dimensional Subsetting Approaches}

One of the simplest methods to visualize high dimensional data is just dropping dimensions and displaying only a subset of these dimensions. Dimensional subsetting methods use algorithmic techniques or user preferences to select a small subset of the dimensions to visualize. The selected subset are then visualized using a 2D scatterplot or any other similar visualization. Allowing users to interactively select the dimensions will support their exploration of the multiple dimensions in the data [126].

However, the success of this approach is dependent on choosing which dimensions contain the most relevant and useful information [126]. As the selection of the dimensions is done prior to displaying the visualization, the information of the most relevant dimensions is unknown at that time. Therefore, the process of determining which dimensions to visualize becomes a significant problem to consider.

\subsubsection{Dimensional Reduction Approaches}

Dimensional reduction methods use computational techniques to transform the data to a lower dimensional space while preserving the relative proximity between data points [34]. The goal is to maintain the relations between the high dimension data in the lower dimension space without adding any new relations that are not originally in the data. Techniques such as principle component analysis (PCA) [72, 55], multidimensional scaling (MDS) [3, 33], and Self Organizing Maps (SOM) [3, 57] are commonly used to transform data to lower dimensions.

These methods project data from the high dimensional space to 2D or 3D space, which allows the use of simple visualization methods (e.g., scatterplots) to display the projected data. The process of transforming the data from higher to lower space provides additional description to the data points (i.e., the principle components for each data point in PCA). 
The final projected space can be used to convey significant features such as clusters and patterns [126]. For example, PCA computes the principal components from the original data, which could be easily used to classify and cluster the data points. Figure 2.4 shows an example of using MDS to visualize gene related chemical compounds in the PubChem database [33].

The end result of using this approach is a visual representation of the data in a coordinate space that may have no obvious geometrical correlation to the actual dimensions, introducing complexity while exploring and understanding the data [34]. Additionally, as the dimensionality of the data increases, the transformation process itself becomes computationally expensive and less efficient. It is harder to preserve the proximity to the actual points and the uniqueness of the mapped points, which means that the mapping could introduce new patterns and relations (e.g., a cluster of points, a specific trend) that do not exist in the data [126].

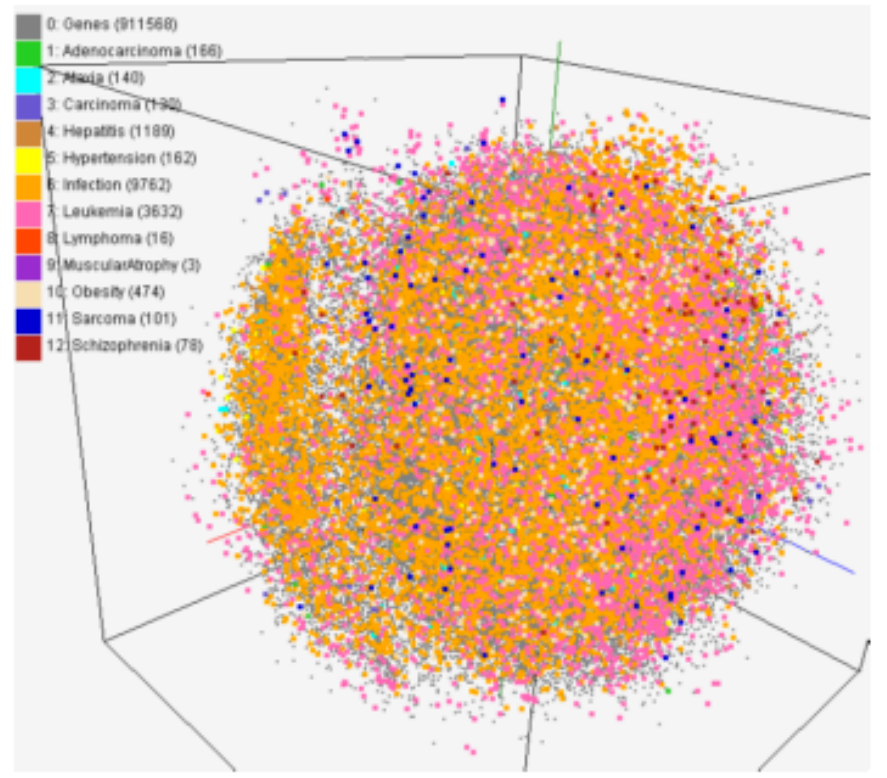

Figure 2.4: Multidimensional scaling example [33] 


\subsubsection{Small Multiples Approaches}

Instead of selecting dimensions or mapping the data to other dimensions, other approaches have been developed to view all the information at once. One of the common approaches in visualization is the use of small multiples to display multiple combination of subsets of the dimensions of the data. The benefit of this method is that it does not hide any aspect of the data, allowing users to compare different views of the data [118].

A scatterplot matrix is an example of small multiples that arranges scatterplots of pairwise dimensions into a matrix [128]. For data with $N$ dimensions, this yields to $\frac{N(N-1)}{2}$ distinct scatterplots, which can be arranged in a $N * N$ matrix (actually, a symmetric matrix with each pairwise dimensions shown twice). Scatterplot matrix systems rely on the simplicity of the scatterplot, allowing users to observe direct relationships between each pair of dimensions. Figure 2.5 a shows an example of a scatterplot matrix to visualize more than 10 dimensions.

The multiple plots allow users to see all the dimensions at the same time, but users need

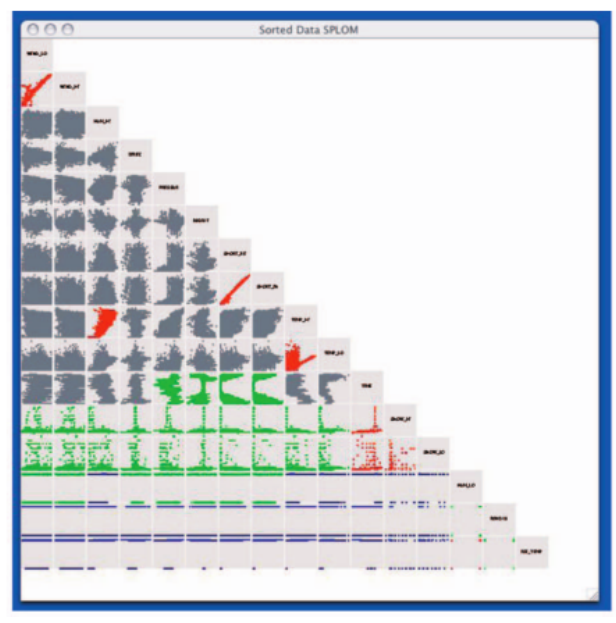

(a) Scatterplot matrix

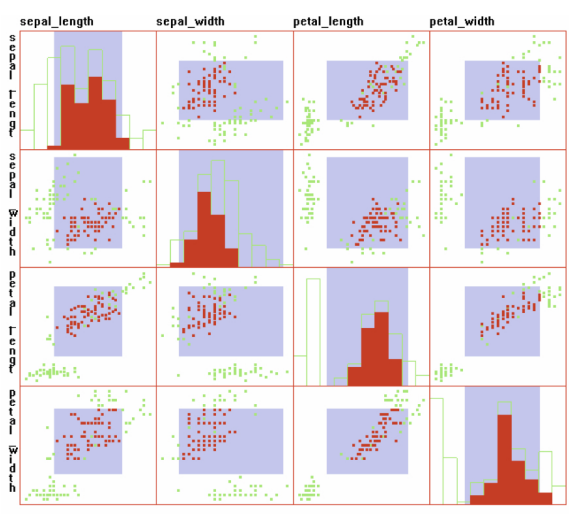

(b) Coordinated interaction in scatterplot matrix

Figure 2.5: Two examples of scatterplot matrix. Figure 2.5a shows coordinations between the different plots [126] and Figure 2.5b shows the use of scatterplot to visualize 16 dimensions [128] 
to interact with the data to understand the relations across these multiple visualizations. Any interaction applied in one view should be automatically applied and linked to other views [126, 68]. Figure 2.5b shows an example of a scatterplot matrix that has a subset of the data highlighted in all the small plots.

Usually the same type of view is used to construct the small multiple visualization. This allow users to compare the different aspects and analyze the data in the same way. However, when displaying data with mix of qualitative and quantitative dimensions, the same view is not the best choice for analyzing each type of data. Depending on the type of data, it may be better to use different visualizations when constructing the plot matrix. Figure 2.6 shows screenshot of such an approach, with different visualizations for qualitative and ordinal dimension in the data [68].

While showing all the dimensions using small multiples has it benefits, it could easily overwhelm users with the number of views. As the number of dimension increases, the dimensions of the matrix increases making it difficult to observe the details in each small view. Additionally, the process of following trends, comparing data, and recognizing patterns across multiple dimensions becomes cognitively tasking.

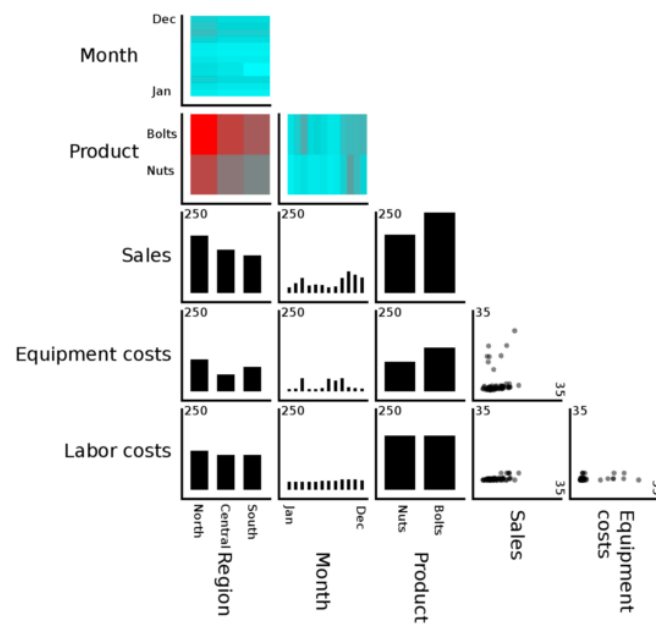

Figure 2.6: General plot matrix (GPLOM) uses small multiples with different types of plots to provide better support for multiple types of data [68] 


\subsubsection{Force Based Approaches}

Several studies show the use of force based layouts to visualize high dimensional data. Force based techniques uses physics principles to layout points on the graph [125]. In these approaches, the visualizations simulate multiple objects that are positioned using multiple springs exerting forces on these objects. The object represents the data points and the forces represent the value of the data in each dimension.

RadViz [66] places $N$ dimensional anchors around a circle, then position the points in the plot based on the forces exerted from these anchors. The distance of the point from the each anchor is proportional to the force exerted (i.e., value of this dimension) on the point. As the points will be laid out depending on the values of each dimension, usually all the dimensions are normalized to values between 0 and 1 before the visualization. The result is a visualization with all the points inside the circle, the location of each point depends on the sum of values in all dimensions.

Force based methods allow for visualization of large number of dimensions, but they have a number of disadvantages. The final arrangement of points could easily introduce patterns, clusters, and trends that do not exist in the data. As the position of the data in the plot depends on the forces from the anchors, the order of dimensions around the plot changes the final location of points inside the plot. As the view will depend on the order of anchors around the plot, the users' insights and understanding of the data will change accordingly. Additionally, even though they provide an overview distribution of the data over the multiple dimensions, it limits users ability to explore the direct correlation between the different dimensions. 


\subsubsection{Glyph Plots Approaches}

The use of glyph plots extends the use of scatterplots from displaying two dimension to displaying a higher number of dimensions. The glyph plot encodes multiple dimensions into graphical parameters in a glyph and then position these glyphs in a 2D plot [55]. For example, one might encode some dimensions of the data to the length property of multiple lines from a centre of a glyph (i.e., in star like shape). The resulting glyph could then be used as a point in the scatterplot or placed discretely on the display space [22]. Figure 2.7 shows an example of a glyph encoding and the resulting plot.

The use of glyphs allows for the encoding of higher dimensional data to simple shapes. The benefit of this method is that it is simple to create the graph by simply encoding data to visual features [22]. Another advantage of using this approach is the use of the scatter plot as the base visualization. As the scatterplot is a very powerful and common visualization technique, displaying glyphs inside a scatterplot supports the user's ability to explore and analyze the data.

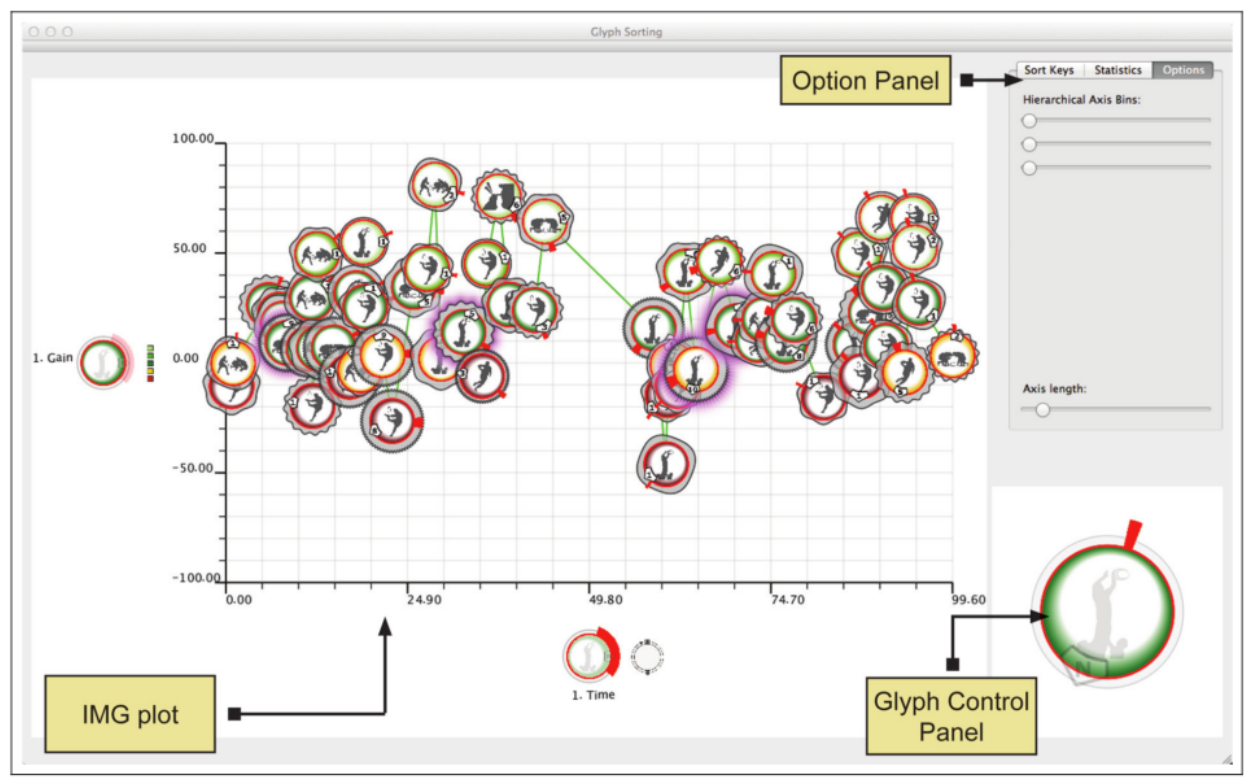

Figure 2.7: Example of glyph plot of high dimensional data [35]. 
Although this approach is based on the usefulness of scatterplots, there is a limit to the number of dimensions that can be visualized using glyphs before they become incomprehensible [35]. The type of the data is another problem to consider. With data that consists of a mix of qualitative and quantitative data types, encoding data to glyphs has to carefully consider the different types of data, making the process of designing a suitable glyph a complex task [22]. Additionally, as the complexity of the glyph increases, the cognitive effort required to learn and understand it increases, limiting the advantages of the approach.

\subsubsection{Parallel Coordinates Plot Approaches}

A fundamentally different approach to the problem is the use of parallel coordinates, where data are represented within a structure that maps each dimension to a parallel axis [69]. Parallel coordinates use parallel axes rather than the orthogonal axes that are expected in the $2 \mathrm{D}$ or $3 \mathrm{D}$ Cartesian coordinates space. With the axes organized in parallel, the data are represented by polylines intersecting these parallel axes at the appropriate locations. A simple example of how a single point in the orthogonal space is mapped to a line in parallel coordinates space is illustrated in Figure 2.8 .

Representing $N$ dimension points using parallel coordinates starts by laying out $N$ parallel axes equidistantly on a plane. After that, each $N$ dimensional point $P_{x}\left(x_{1}, x_{2}, x_{3} \ldots x_{n}\right)$ is represented using a series of line segments $L$ connecting between each two consecutive
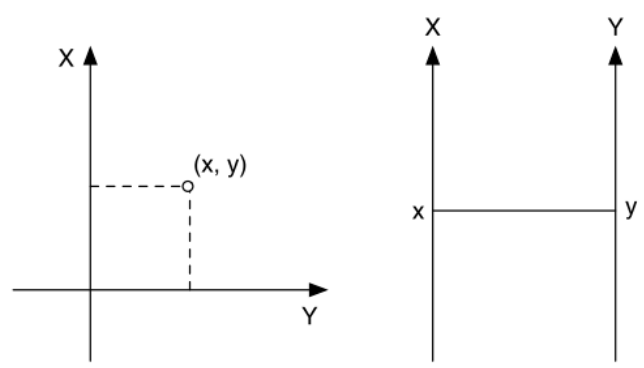

Figure 2.8: Mapping a point from the 2D space to the parallel coordinates space [111]. 
dimensions $L_{1}\left(x_{1}, x_{2}\right), L_{2}\left(x_{2}, x_{3}\right) \ldots . . L_{n-1}\left(x_{n-1}, x_{n}\right)$. The benefit of this configuration is that the number of dimensions that can be visualized is only limited by the horizontal space available (i.e., if the parallel axes are organized vertically ) [111]. Figure 2.9 shows an example of two 6D points visualized using the parallel coordinates. Depending on the values of the data in each dimension, the scale of each axis could be different.

With high dimensional data, the goal of visualizing data is to enable the users to recognize and identify the different patterns and trends in the data. For reasons of simplicity of the explanation, a simple $2 \mathrm{D}$ dataset is used to illustrate how to recognize the various patterns and trends in parallel coordinates. Figure 2.10 shows examples of the common features in the data and how they are visualized in both an orthogonal scatterplot and parallel coordinates. A simple linear relation between the two dimensions is represented in the parallel coordinates by a sequence of non crossing lines (Figure 2.10a). An inverse relation can be easily identified inside the parallel coordinates by multiple crossing lines (Figure 2.10b). A cluster of points can be recognized in the parallel coordinates by a group of close intersecting lines (Figure 2.10c).

Figure 2.11 shows an example of high dimensional data visualized using the parallel coordinates, where some patterns and relationships are clearly visible. The main advantage of using parallel coordinates rather than the orthogonal coordinate system is that it is

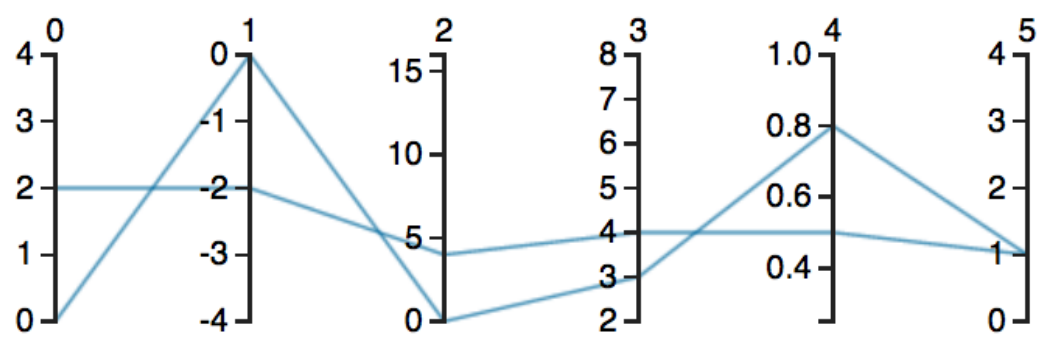

Figure 2.9: A two 6D points mapped in parallel coordinates. The plot represents the two points $[0,0,0,3,0.8,1]$ and $[2,-2,4,4,0.5,1]$ 

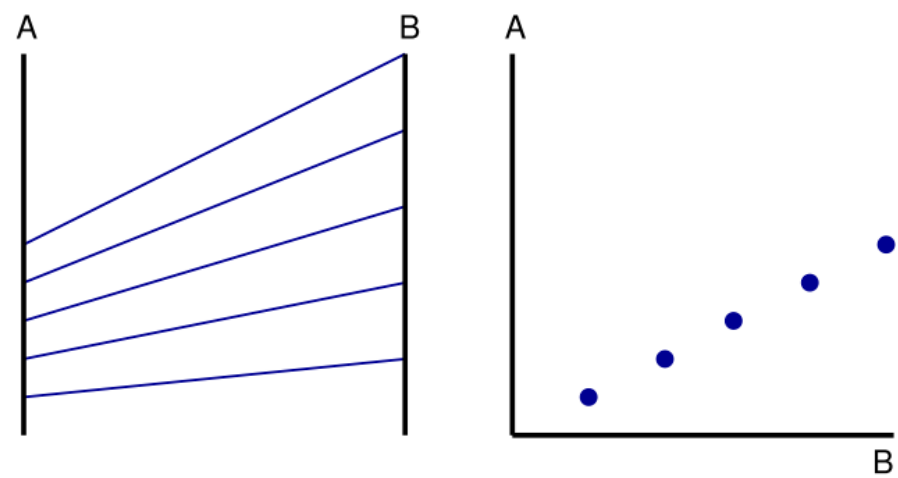

(a) Linear relation
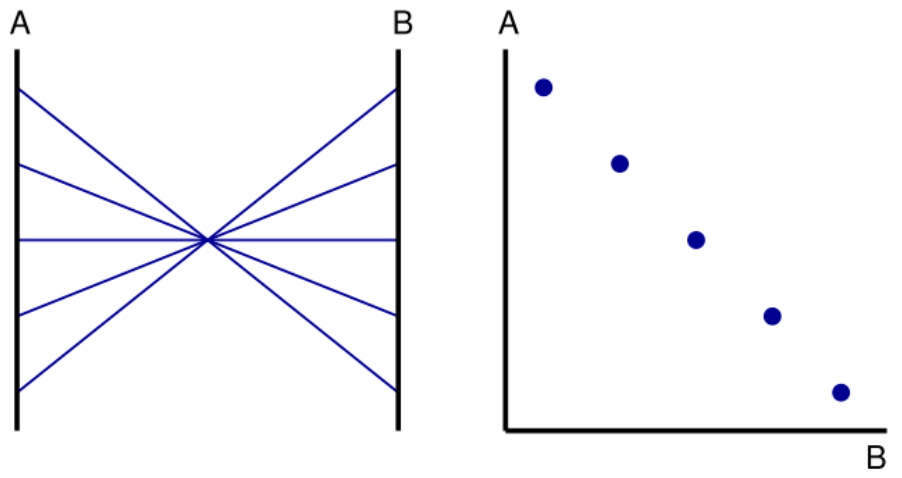

(b) Inverse relation
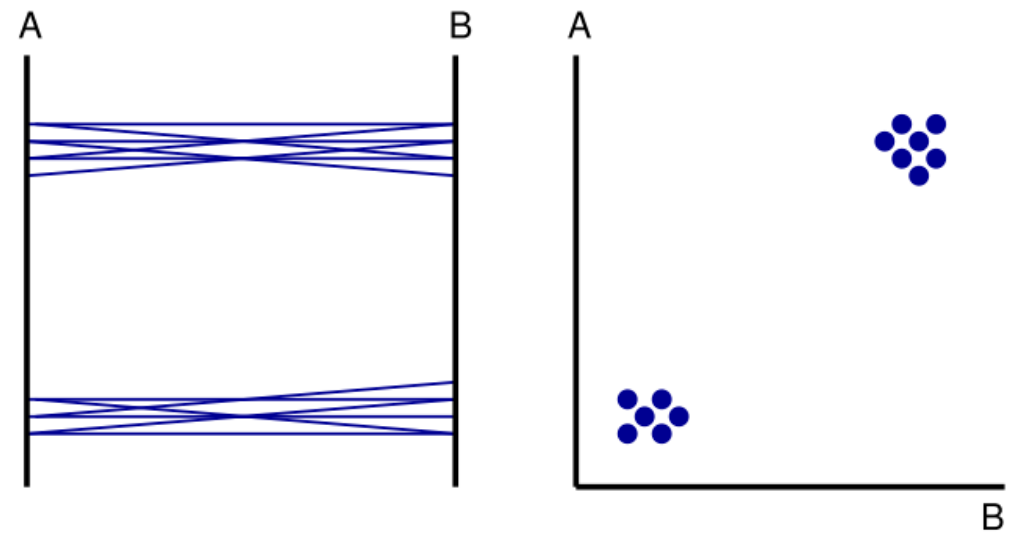

(c) Multiple clusters

Figure 2.10: A scatterplot and parallel coordinates plot representing the same features in the data [71]

flexible and scalable with respect to the number of dimensions visualized. A second advantage is that it provides a view of multiple dimensions at once, allowing users to gain an overview of the data across all dimensions. This overview of the data permits users to 


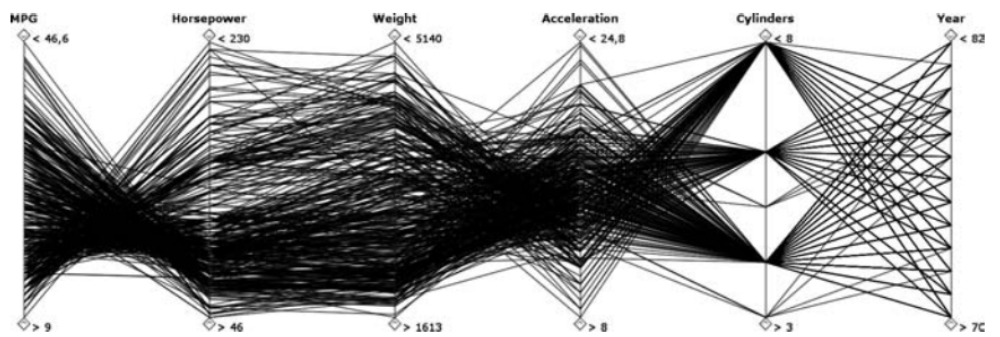

(a) Each polyline is rendered completely opaque.

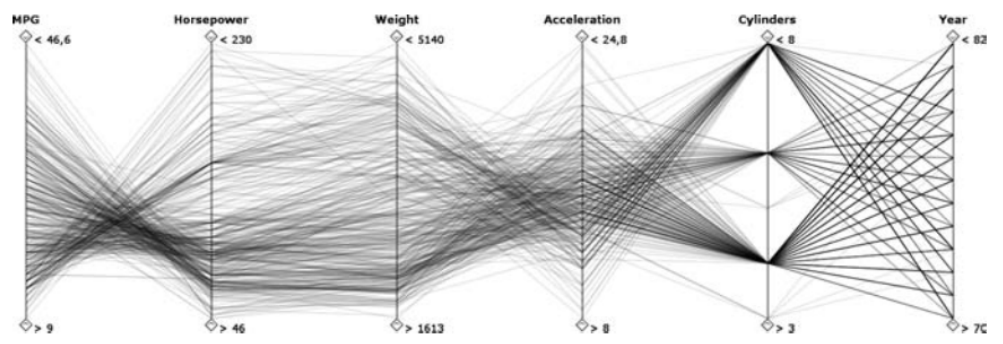

(b) Each polyline is rendered with an opacity of $15 \%$.

Figure 2.11: A car dataset visualized using parallel coordinates. The figure shows the effect of changing opacity based on the density of the data [71].

identify patterns over multiple dimensions at the same time.

Using parallel coordinates, the arrangement of the dimensions may seem strange at first. However, multiple user studies $[60,73,84,111]$ have shown that, parallel coordinates are easy to learn and users are able to quickly gain an understanding of the data. With the first use of parallel coordinates, users learn how to read the visualization and understand how the points and patterns are transformed from the familiar Cartesian space to the parallel coordinates.

To further support users in their exploration and analysis of the data, users should be able to interact with the data in parallel coordinates. Several interaction techniques have been used in parallel coordinates to explore and manipulate the visualized data [60, 111]. Filtering, selecting, and navigation techniques are used to explore different subsets of the data. The most common method to filter data in parallel coordinates is brushing over the axes to select points that fall between the range of values selected. However, several other types of brushing techniques have been introduced such as angular brushing [58] and 2wD 
brushing (i.e., rectangle brushing over the lines between the coordinates [91]). While filtering the data will remove the excluded the data from the view, selecting data points will highlight these specific points by either changing their colour or fading the rest of the data. Exploration of the data is also possible using navigation techniques such as: zooming in, zooming out, and panning through different subsets in the dataset. Other interactions include changing the display by manipulating the encoding or reconfiguring the data [60].

Even though parallel coordinates have multiple advantages, there are also a number of important limitations. When a large number of data points are shown using parallel coordinates, overplotting may occur, resulting in visual clutter. A simple solution to the problem could be addressed by interactively highlighting the data points using brushing, highlighting, or another selection methods [134].

Another solution to the problem is density plotting by drawing thinner or less opaque lines, which results in darker regions at high density locations and lighter regions at low density locations [71]. Others have tried to summarize or abstract the data to provide an overview of the high volume of the data (see Figure 2.12) [97, 133]. Clustering and outlier detection algorithms have also been used in order to reduce the amount of the shown data, and to highlight those data points that are different from the norm [57, 134].

Overplotting also occurs when multiple data points intersect an axis at the same location, which introduces ambiguity. This could be addressed by replacing the polylines with curves or density functions, both of which make it easier to perceive and follow the data points through the parallel coordinate structure [71, 60].

Also due to overplotting of data points in parallel coordinates, grasping the distribution of the data and other statistical properties is difficult. Several approaches have addressed this problem by adding statistical information to the parallel axes [62]. However, the extra data on the axis but may further contribute to the complexity of the display.

While parallel coordinates display high dimensional data, finding relations across the 


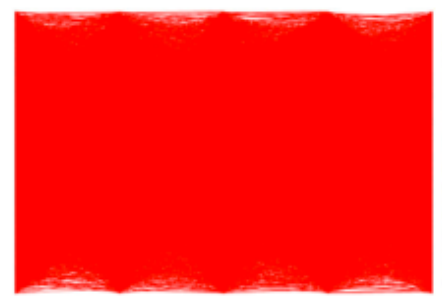

(a)
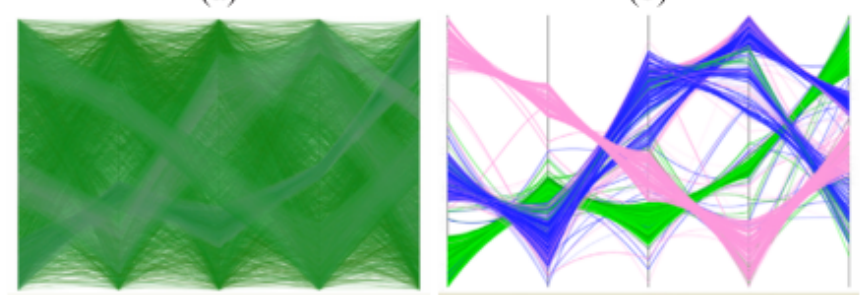

Figure 2.12: Example of visual cluster [134]

large number of dimensions is not a trivial task. Direct relationships between pairs of dimensions are easily noticed for adjacent coordinates, but relations between distant dimensions are not clearly identified. One of the powerful interactions employed in parallel coordinates is interactively reordering the coordinates. By reordering the coordinates, users are able to examine different relations and gain new insights. Automatic reordering algorithms use correlation analysis between dimensions to arrange the coordinates [60, 111]. Other systems allow users to manually rearrange dimension to support exploration of the data according to the users' task.

When a particular dimension of the data represents discrete qualitative values, the ambiguity and overplotting problems become even more acute. Parallel sets provide an alternative for visualizing such categorical data, using ribbons between the coordinates to represent the frequency of each category (see Figure 2.13) [79]. However, combining this with traditional parallel coordinates when the data includes a combination of qualitative and quantitative data is not feasible. 

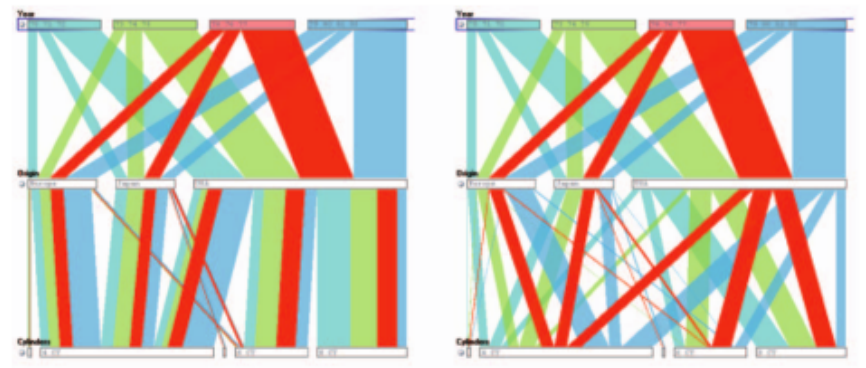

Figure 2.13: Parallel sets [79]

\subsubsection{Hybrid Parallel Coordinates Approaches}

Several systems tried to combine the power of parallel coordinates with other visualization techniques. Force directed parallel coordinates tries to extend the interactive capability of parallel coordinates by applying force directed interactions [125]. Others proposed coordinating between multiple high dimensional visualizations at the same time. Examples of such systems include combining the RadViz [17], or reorder matrix [110] with the parallel coordinates.

These hybrid approaches propose benefits from integrating the different techniques. The linking between the multiple views could provide deeper insights, but it is cognitively challenging to interact with multiple high dimensional views especially if the visualizations are conceptually different. For example, in the parallel coordinates matrix [59], the system displays multiple parallel coordinates in a matrix structure to show the different axes orderings. The resulting visualization is much more complex than traditional parallel coordinates, requiring significant cognitive effort from the users. Additionally, as the multiple the different techniques will be displayed simultaneously, such systems are computationally less efficient than each visualization by itself and consume more screen space. 


\subsection{Geotemporal Data Visualization}

Recent advances in GPS technologies have increased the access and collection of geographical information in nearly every field. This growth and availability of geospatial data are exploited in a wide range of scientific and industrial applications [9, 85, 86]. The large volume of geographical data and diversity of the sources increases the need for tools to support analysis and exploration of geographical data.

Geographical databases combine a wide range of diverse spatial and non spatial attributes to describe geographical locations. The spatial attributes describe the geographical information about a particular location, such as the exact latitude and longitude of a specific village. The types of vegetation, the population of the village, and percentage of employment are examples of the non spatial attributes. Due to the diversity of these attributes, understanding and making sense of geospatial data is a challenging problem.

While displaying data over the map allows for relating the data to the important geographical context, the choice of what is displayed and how it is displayed over the map affect the types of insight users get from the visualization [104, 126]. Various visual forms have been developed to display geographical data, such as dot maps, choropleth maps, and cartograms maps [104]. As each of these visualizations provide a different viewpoint of the data, selecting the visualization to use depends on the type of geospatial data. Dot maps are better suited for geographical point features, where a shape or symbol is placed at the location of the data. Choropleth maps are mainly to visualize area features, where each different land area is shaded with values depending on the data presented [104]. Cartograms distort the map depending on the values in the data, which allows for better representation for some statistical information such as population. These maps resize the regions according to the values, but too much distortion makes relating to the geographical context difficult [126]. 
Dot maps display the non-spatial data by encoding them into visual properties of shapes placed on the map. The advantage of this approach is that it allows for visualizing various non-spatial attributes without losing the geographical context [126]. Displaying multiple attributes requires encoding them into the visual properties of the shapes on the map. However, as the number of attributes increases, there are a limited number of visual properties to use without complicating the view. In order to explore and analyze the data, users should be able to easily choose the information to display, manipulate the view, and correlate between the different forms and data presented [104].

Additionally, adding a temporal aspect to the geospatial data is another challenging problem. The first issue is that temporal data provide another aspect to consider when visualizing the data. Another problem is that geotemporal data have different scales and relations between the geospatial and temporal aspects of the data [13]. In order to make sense of the such data, users have to be able to explore and analyze the diverse factors and their relations to space and time. Supporting visual analysis of geotemporal data is a challenging problem that involves dealing with multiple tasks. While there are different geospatial (i.e., area or path) and temporal data types, the focus of this research is geospatial point data and single event temporal data.

Several approaches have been proposed to support interactive visual analysis of geotemporal data. Geographic Information System (GIS) is one of the earliest and most commonly used technique to visualize and analyze geotemporal data [105, 113, 130]. Most GIS systems allow users to add layers to a map in order to show the spatial distribution of the different factors. Utilizing GIS to detect relations within the data is a challenging process, since it involves visually merging and comparing multiple layers [130]. When there are a large number of dimensions to analyze, this process of selecting the layers and visually comparing the data is inefficient and cognitively taxing. 
Another approach is using two coordinated visualizations, one to represent the temporal aspect of the data and the second to represent the geospatial aspect of the data. A temporal view such as a timeline may be used to display the temporal information. A second view may show the geographical information on a simple map visualization such as a dot map [70] or a choropleth map [57] depending on the type of geospatial data. This allows the user to observe and inspect the different aspects of the data at the same time. However, this approach relies on the users interacting with the visualization to support their understanding, comparing, and connecting between the two aspects of the data. To support exploration and analysis of the geotemporal data, the visualization must coordinate between the different views and allow users to interactively manipulate the information displayed on the map.

Rather than simply placing a glyph at the location, this glyph can also be used to represent an aggregation of the temporal data [9]. Displaying the temporal data on the map, allows users to see the change over time for each location. Figure 2.14 shows a different glyph for each week of traffic data overlaid on the map. With the support of interaction techniques such as filtering and selection, users are able to analyze and understand complex geotemporal patterns displayed on the map. However, using this method to display the temporal aspect limits the ability to show different attributes and aspects of the data on the map. Thus, it limits the number of multivariate attributes that could be visualized and analyzed.

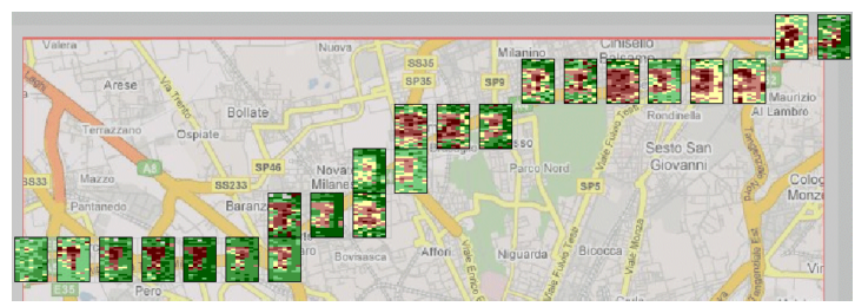

Figure 2.14: Example of showing a glyph to represent temporal data on the map [9] 
Another method specifically for geotemporal data is the space-time cube, where location is represented in the map dimensions, and time is represented in the third perpendicular dimension (see Figure 2.15] [80]. The approach has been used to visualize trajectory and movement data, as it gives a clear view of the sequence of data over both space and time [10, 52]. Due to the nature of the 3D views, the approach can not handle a large number of dimensions at the same time before visualization becomes crowded and occlusion is a problem.

Small multiples have also been used to visualize geotemporal data by using multiple small maps to show the change of geospatial data over time. It allows users to see the trend of the data and compare the data across multiple views. The approach is used to show the flow and the trend of the data from one location to the other by display a small map for each time period [108]. The change in the data or trajectory data could be shown using arrows over the map. While the approach provides a tool to compare the trends over the multiple views, it limits the amount of details that could be clearly shown on the small views (see

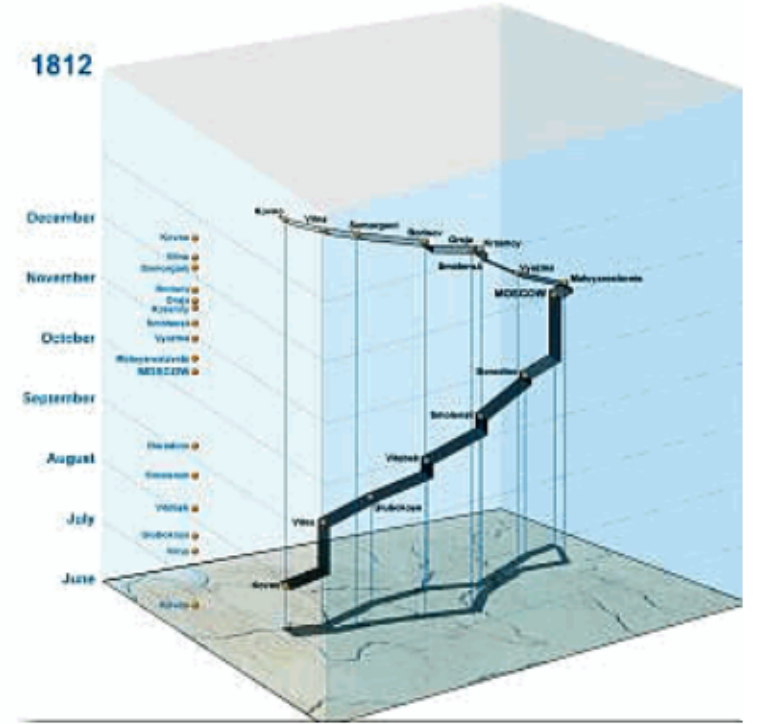

Figure 2.15: Geospatial location is represented on 2D plane and the time is presented on the third dimension [80] 
Figure 2.16. As the small multiples each represent specific time periods, the approach limits users' ability to analyze complex temporal patterns.

\subsection{Multiple Coordinated Views}

When data consist of both high dimensional attributes and geotemporal aspects, a single visualization method may not be adequate to support exploration, comparison, analysis, and knowledge discovery across the different aspects of such complex data [103, 106]. Multiple views provide users with alternative viewpoints or perspectives of the data. These multiple viewpoints allow users to gain different insight and understanding of the data. As a result, the use of multiple visualizations can support the exploration of the complex aspect of the data.

Multiple coordinated views (MCV) is defined as the use of multiple views displaying different aspects of the same dataset at the same time. As the multiple views will show different aspects of the data, users must be able to link between the same data points in these different views. One approach to link these views together is coordinating the interactions

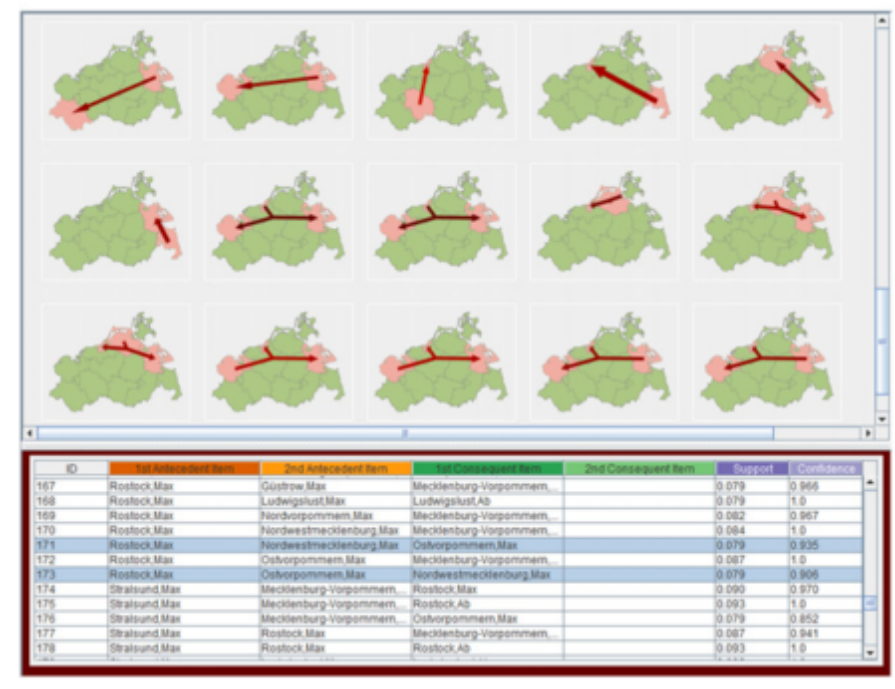

Figure 2.16: Example of small multiples showing multiple maps to show the flow of the data [108] 
between the views. Any interaction applied on one view (such as selection), is automatically applied to all other views in the system. Coordinating between the multiple views is essential to understand the relations between the data presented on the various views.

With the multiple views showing the data, the coordination between the views allows the user to search the data, identify patterns and trends, and make sense of the data presented. Several interactions have been used in MCV systems to link between the views [104]. Selecting and filtering are examples of the techniques used to select subsets of the data. Navigation techniques like pan and zoom allows users to navigate through the data. Encoding and re-configuring interactions are techniques used to control the appearance and the representation of the data [104]. In order to effectively link between the different views, all these interactions must affect all the views and the data equally. For example, selecting a set of point in one view must be reflected in all the other views by automatically selecting or highlighting the same set of points.

Over the last decades, several types of MCV systems have been developed to support visual exploration. Roberts [103, 104] reviewed and classified the MCV approaches into side-by-side views, overview details, context+focus, difference views, and small multiples. To represent the different aspects of the data, side-by-side view systems use multiple views beside each other. Such systems usually use different views to represent the various aspects of the same data [57]. An example of this approach is VIS-STAMP [57], where a combination of multiple choropleth maps, re-orderable matrix, and parallel coordinates are used to visualize complex geotemporal data.

Overview details and focus+context are similar as they both present two types of information: one view displays an overview of the data while the other view provides the details [94, 108]. However, a lot of focus+context systems use a single view to display context and the focus is achieved using distortion of the context view [104, 126]. The benefit of this approach is that users have two levels of details of the data simultaneously, 
supporting the exploration of various aspects of the data. This permits users to explore the details of the data while still retaining visual awareness the overall organization of the data. For example, showing an overview of the data on the map and having the detailed table of the data below it [57, 88, 108]. Such systems allow users to see the details of the data in the tabular view while maintaining the awareness of the geographical distribution of the data.

Difference view systems focus on allowing users to compare between the data by providing views that explicitly show the difference. This approach focuses on supporting users to identify the differences or changes in the data. GTDiff computes the changes over specified timeframes and visualizes these differences using small maps, allowing the users to see at a glance where and when the data have changed [65].

The final approach for MCV is the use of small multiples views to show small visualizations of different dimensions or aspects of the data. The approach visualizes different aspects of the data on multiple small views usually of the same visualization type (e.g., multiple scatterplots in a scatterplot matrix, or multiple choropleth maps) [104], allowing users to follow the trends and compare multiple views of the data at the same time [57, 65, 108$]$.

From a geospatial analysis perspective, the coordination between parallel coordinates and geovisualization approaches have been studied for many years [7, 50]. However, there remains a shortage of geovisual analytics systems that support interactive analysis of high dimensional geotemporal data [12]. Furthermore, linking these approaches within an integrated analysis of qualitative non-spatial attributes remains an open problem that we wish to address in this research.

\subsection{Environmental Data Visualization and Visual Analytics Approaches}

Due to human development and natural hazards, the natural equilibrium and resilience of the environment and ecosystems is continuously changing [95]. Additionally, the huge 
demand humans are placing on the environment is causing numerous environmental problems [14]. A wide range of factors are impacting the environment; understanding the significance of these factors plays a vital role in addressing environmental problems. There are multiple biological, ecological, economic, and environmental factors that influence these environments and ecosystems. These factors are heterogeneous with multiple interrelations, and with important spatial and temporal aspects at different scales. In order to understand environmental problems and supporting better ecosystem management, researchers need to synthesize large amounts of information and analyze the complex relations in the data [82, 14, 113].

In the last two decades, a number of researchers have explored various environmental analysis tools [4]. Several assessments and analytical techniques (e.g., Strategic Environmental Assessment [98], Life Cycle Assessment [37], and Cost-benefit Analysis [20]) were introduced to analyze the impact of the different factors on the environment [4, 46, 67]. These tools allow researchers to assess the environment and the impact of factors used in the analysis. Similarly, several frameworks have been proposed to guide researchers in understanding diverse environment factors. For example, the DPSIR framework [15] (i.e., Drivers, Pressures, States, Impact, Responses) is used to describe the interaction between society and the environment. The framework has been adopted by the European Environment Agency [42] and it has been widely used to assess and manage the environment in various socio-environmental problem settings [15, 32].

With the increase need for improving current environmental decision support systems, researchers investigated various multi-criteria decision making frameworks. These frameworks use multiple performance measures and analytical methods to guide the creation of scenarios that allow for the exploration of different decision alternatives [6, 74]. Investigating these alternatives provides decision makers with the means to understand the impact of 
different factors in the environment. The goal of these approaches is to explore how scenarios and factors influence the generated alternatives. However, they do not provide tools to explore and analyze the actual environmental data, limiting their value if the goal is to understand the relationships within the data. While these approaches are useful for studying the impact of specific environments, they have limited abilities if you want to analyze geotemporal patterns and trends.

Geographic information systems have been commonly used in analyzing environmental systems, supporting the visualization and analysis of geotemporal environmental data [105, [113, 130]. As previously discussed, GIS visualizes multiple factors by displaying layers of information over the geographical locations. Understanding the relations between different attributes involves merging and running several optimization algorithms on these layers. With multiple operations and analyses provided in current GIS commercial systems, various GIS-based approaches have been developed to support environmental analysis [113, 121]. As analysts need to make sense of the multiple layers of data, the analysis of numerous diverse factors is still a challenging problem in these systems.

To address these shortcomings, some have proposed decision support systems for environmental problems that focus on engaging decision makers in the analysis process by adopting an interactive visual analytics approach [74, 87, 112, 129]. These approaches use visual analytics to extend current environmental analysis tools. An example of these approaches is a system to explore species distributions and their relationship to environmental data by extending traditional GIS systems using a taxonomy viewer and multivariate tabular visualization [130].

Others have developed similar methods to support exploring among environmental data, but with a focus on specific environmental problems or domains [78, 112]. A visual analytics system was proposed to explore and analyze elevation changes in coastal environments over time [114]. Another system uses multiple coordinated views to explore hydrodynamic 
simulations of coastal environments [53].

These approaches allow researchers to explore and make sense of complex environmental data, but they suffer from one main disadvantage most of these systems are domain and task specific. With the large number of diverse databases and problems that need to be addressed, it is time consuming and unfeasible to develop a visual analytics approach for each problem. While there is a need to develop visual analytics systems to support analysis of the complex environmental data, the ability to generalize these systems to multiple datasets is essential. Additionally, a detailed evaluation of the analysis of multiple environmental datasets using visual analytics systems has not been investigated. 


\section{Chapter 3}

\section{Geo-Coordinated Parallel Coordinates}

With the increasing amount of data collected and stored, the ability to explore and understand this data is an essential step for effective decision making. While understanding massive datasets are a demanding problem, understanding high dimensional heterogeneous geotemporal data is also challenging. Visual analytics provides an approach that combines automated analysis and computation with interactive visualization to support understanding and reasoning about complex datasets [13, 75].

The fundamental objective of this research is to support data analysts to discover new knowledge and make informed decisions about the data by providing them with tools to explore and understand high dimensional heterogeneous geotemporal data. A visual analytics approach is employed to support exploring and analyzing complex patterns and trends within such data. The main purpose of this work is to support reasoning and exploring among the data, with a focus on five main design goals:

Support for high dimensional geotemporal data The system should provide the means to visualize and interact with high dimensional geotemporal data. Both the visualization and interaction have to facilitate identifying direct and indirect relations between the high dimensional data and geotemporal aspect of the data. 
Support for different types of data The system must be able to support a mix of quantitative, qualitative, ordinal, spatial, and temporal data. This includes the ability to identify, visualize, and handle different data types automatically.

Easy to communicate To support decision making and facilitate explaining patterns in the data to officials and non-technical users, the system has to be easy to explain. The visualizations must be easy to interpret and communicate with other parties. The goal is to assist in information exchange and communicating insight gained from the system.

Flexible The system should easily be used to visualize different datasets from various environmental studies or regions. Each environmental study usually has its own set of parameters and dimensions. The aim of the system is to provide a tool that can be used in various studies to support the analysis of high dimensional geotemporal data. This flexibility has to extend to various other features of the system. With a high number parameters in environmental studies, the system should enable analysts to focus on a small subset or provide an overview of the whole dataset.

Focus on interaction In general, the main tasks for any analysis is to synthesize information, explore the data, derive insight, detect the expected, and discover new unexpected knowledge [75]. One goal of this work is to concentrate on providing multiple interactions to support these tasks and enable exploration and understanding of the data. The interaction of users with the data should provide a dialogue with the data and allow analysts to take part in the analysis process [103].

This Chapter describes the design and the implementation details of this visual analytics system, called Geo-Coordinated Parallel Coordinates (GCPC). GCPC uses multiple coordinated views to show, examine, and explore high dimensional heterogeneous geotemporal data. It has been designed to enable the interactive analysis activities described in Keim's 
visual analytics mantra: "analyze first; show the important; zoom, filter, and analyze further; details on demand" [75]. Following this mantra, the initial view of the system shows the main aspects in the data after they have been preprocessed. Multiple coordinated views allow the user to investigate the data using filters, zooming, and other interactions across the different visual representations. Additional analysis tools are provided to explore direct correlations between factors, analyze outliers, and examine details about the statistical distribution of the data. A detail window and table view provides users with raw data and detailed information on demand.

This remaining of this Chapter presents the key features of GCPC in detail. Section 3.1 provides an overview of the system architecture and the core modules. A system block diagram is used to illustrate the connection between the different modules with a brief

description of each module. Next, the details of each module are explained in Section 3.2. Finally, Section 3.4 discusses the system with respect to the stated research challenges and design goals.

\subsection{System Architecture}

To support the analysis of the multiple aspects of high dimensional heterogeneous geotemporal data, GCPC consists of multiple visualizations with various coordinated interactions. The core of the system consists of two tightly integrated views: a parallel coordinates plot and a geovisualization. The integration between the two views allows the system to represent high dimensional, heterogeneous, temporal, and geospatial aspects of the data simultaneously. An additional scatterplot view allows analysts to interactively investigate direct correlations between different dimensions. Figure 3.1 illustrates the architectural structure of GCPC.

Prior to using any dataset in the system, the data have to be preprocessed to a format 


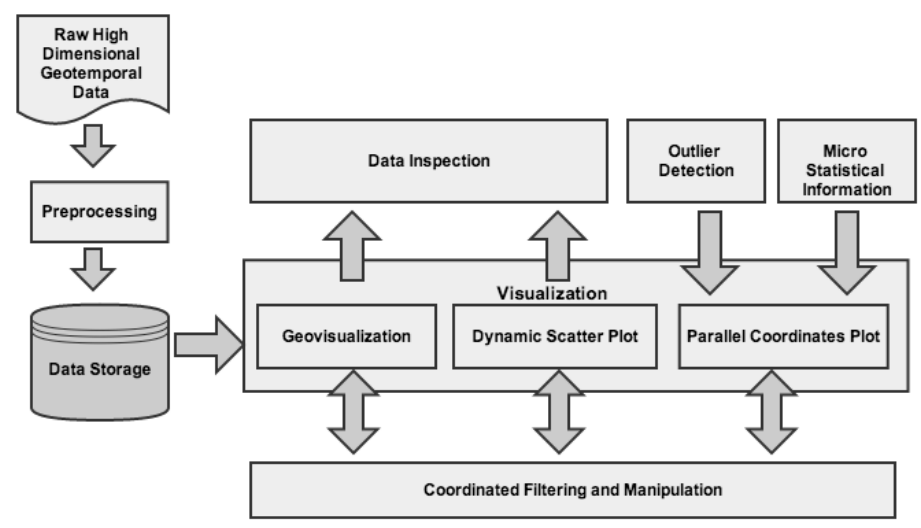

Figure 3.1: The architectural diagram of GCPC

readable by the system. This step re-configures the data into a tabular format and converts raw data to more useful configurations (e.g., converting location information from text form to latitude and longitude). The results from this one time preprocessing phase are then stored in a database system, which will be used to introduce data to the system.

Once the data are stored in the database, the next step is to load the data from the database to the visualization. There are three main views: the parallel coordinates, the geovisualization, and the scatterplot. The high dimensional temporal, qualitative, and quantitative aspects of the data are visualized using the parallel coordinates. The geospatial data are presented by the geovisualization. The scatterplot allows the user to view direct and indirect correlations between different dimensions. The coordinated filtering and manipulation module is responsible for linking and connecting any filtering and interaction applied in one view to all the other views.

The system includes multiple analytical components to provide automatic analysis for the data. The first tool is the computation of statistical information for each dimension visualized within the parallel coordinates view. The second component performs outlier detection to allow users to investigate outliers in more detail or remove them from the analysis. The full details of the data are provided by the data inspection module, which allows users to drill down to details of the points to gain understanding of the context of 
the data.

The system was developed in three tier system architecture: data layer, logic layer, and presentation layer. In the data layer, MongoDB [1] was used to store and handle the data requests. PHP [56] was used to implement the logic tier in the system, running within the Apache web server [48]. This layer is responsible for preprocessing the data, responding to client requests, connecting to the database, and handling data manipulation. The last tier is the presentation layer, which was implemented using JavaScript [47]. This layer represents the interface and it is divided into the different components in the system (see Figure 3.1). The interface was developed using the Data Driven Documents (D3) library [23] as the core. Existing parallel coordinates [29] and geovisualization [2] plugins were used and extended to add novel visual and interactive features. After the initial page is loaded, AJAX is used to communicate between the interface layer and the web server.

\subsection{Geo-Coordinated Parallel Coordinates}

GCPC is composed of multiple views that are linked and coordinated with multiple interactions. A parallel coordinates plot is used to visualize the high dimensional heterogeneous data. From within the parallel coordinates view, micro-visualization of statistical information and encoding controls are displayed on top and bottom of each coordinate, respectively. The parallel coordinates are tightly coupled with a geovisualization that displays the geospatial data using either a dot map or an aggregated hexagonal map. Figure 3.2 shows a screenshot of the system.

The scatterplot view allows analysts to interactively investigate correlations between pairs of factors. The user could use the controls below each coordinate of the parallel coordinates to choose the dimension visualized in the scatterplot. Data inspection is provided whenever the user selects a data point. The system includes a table view to support a 


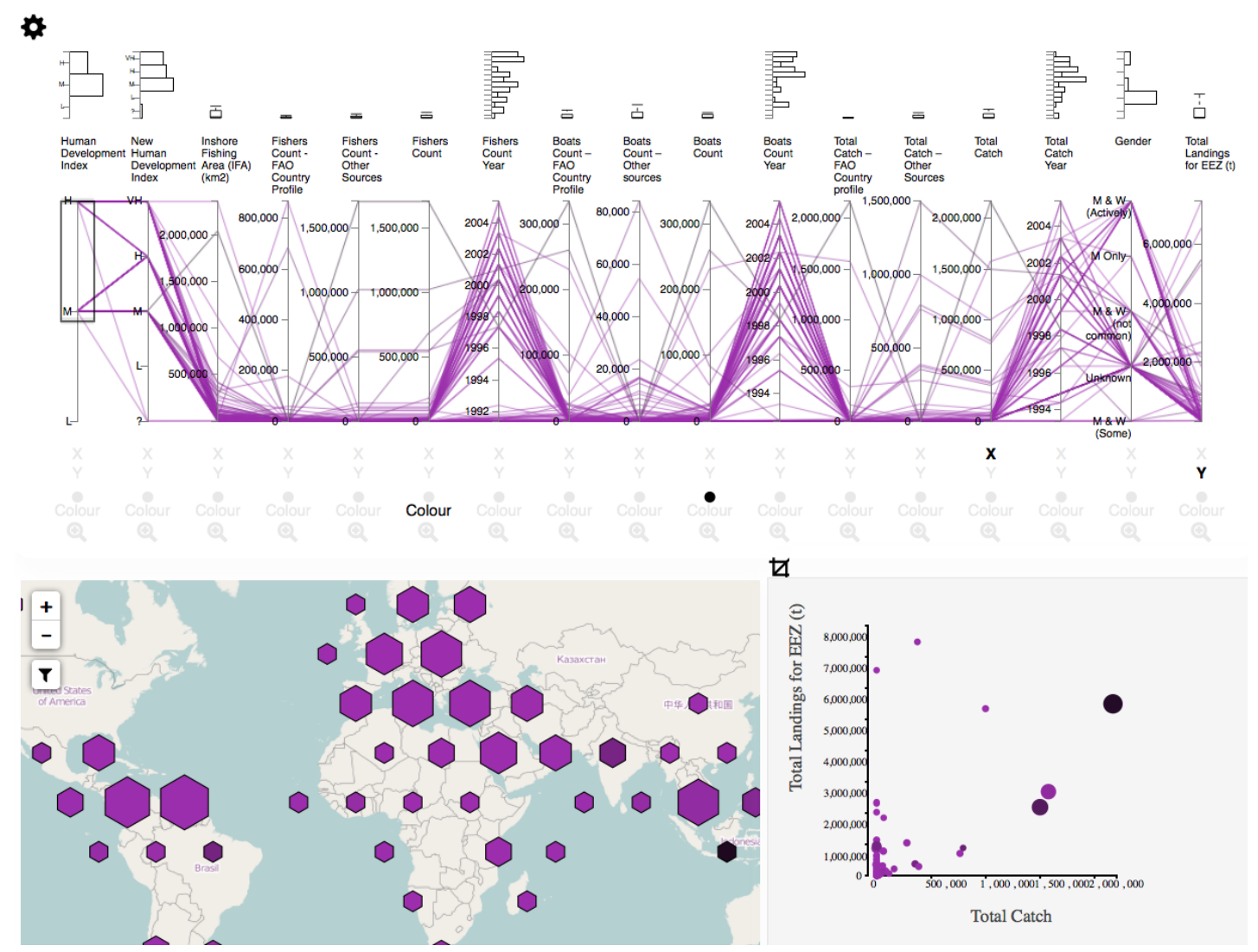

Figure 3.2: The main view of the GCPC consists of parallel coordinates, microvisualizations of the statistical properties of each dimension, interactive controls for configuring the visualizations, a geovisualization of the geospatial distribution of the data, and the investigative scatterplot. Here, the data is filtered for specific values on the first dimension and coloured based on the values in the sixth dimension.

detailed inspection of multiple data points at the same time.

To support exploration within the data, one of the main features in GCPC is the tight coupling between the multiple views using a highly coordinated interactions. To allow users to explore the data in multiple ways, the core modules are linked through various coordinated interactions: navigation and zooming, filtering, highlighting, and reconfiguring the visualization.

Navigating through the data is supported on all views by allowing user to pan and zoom into a subset of the data. Filtering and selecting subsets of the data are also supported in all visual representations. While navigation and filtering are used to focus on a subset of the 
data, allowing users to change how the data is visualized also supports the understanding of different patterns and features in the data. The system allows users to reconfigure the visual encoding of the data based on any dimensions.

\subsubsection{Preprocessing}

Preprocessing is a fundamental step when one is dealing with heterogeneous raw data from multiple sources. Raw data is often noisy, inconsistent, redundant, and erroneous, therefore they normally need cleaning, transforming, and normalization before any analysis step. With the goal of supporting the analysis of various datasets, we need to preprocess the data to clean and format it before introducing it into the system.

To clean the data, a semi-automated process is used to check the data for inconsistent values and categories (e.g., using different words for the same category, different units for the same numerical data). In some cases, categorical data could be represented in the data by numerical values (i.e., 0 and 1 instead of yes and no questions). This representation will limit users' ability to analyze and understand such data. Thus, based on the meta information provided with the data, such numerical values are mapped to the correct categorical data.

A PHP script has been developed to implement this process, which takes the raw data and maps it to the correct categorical form. Data can be introduced to the script in various formats. Comma separated values (CSV) could be used to input the raw data values. The script requires that all data points have the same number of dimensions and that the geographical location dimensions are clearly marked (i.e., the name of the dimension has "geo" for example geolatitude or geoaddress). The data must include a code book that contains descriptions and the meaning of the categorical values for each dimension. This is used to convert the values in the data to the correct category from the code book. The description of the dimension and the type of data is then extracted and saved in the database. 
After the cleaning process, the data is transformed into a tabular format. Next, qualitative dimensions of the data are converted into categorical or ordinal data if the data represents a specific order. Descriptive statistics about the quantitative dimensions are computed to speed the initial processing of the data. When no metadata is provided with the data, the type of each dimension is extracted from values and meta-data of the data are created and stored.

Normally, geotemporal datasets have the exact geographical coordinates provided, but some datasets just have a textual reference to locations (e.g., Canada or San Francisco Bay). This text representation of the locations has to be transformed to exact geographical coordinates. Therefore, the final preprocessing step is to run a geo-encoding algorithm [38] to encode location information in the data to the exact geographical information and save it to the database.

\subsubsection{Parallel Coordinates Plot}

A fundamental requirement of the system is to visualize and analyze numerous heterogeneous dimensions. Parallel coordinates plot is a visualization method that has multiple advantages for analyzing high dimensional data [60]. Parallel coordinates use parallel axes rather than orthogonal axes to organize the dimensions. The data are represented by lines intersecting these parallel axes at the appropriate locations.

Due to the scalability of parallel coordinates with respect to the number of dimensions, GCPC utilizes parallel coordinates as one of the main visualizations of the system. Another advantage of employing parallel coordinates is that it permits users to follow the trend of the data over multiple dimensions at the same time. Additionally, the use of parallel axes in the view facilitates adding or removing dimensions dynamically.

To support heterogenous data in GCPC, the parallel coordinates is used to visualize the quantitative, qualitative, ordinal, and temporal data. Numerical and quantitative values are 
rendered in a continuous scale over the coordinates. Qualitative data are represented in the parallel coordinates with discrete points on the coordinate, which is ordered alphabetically if the data is categorical or ordered appropriately if the data is ordinal. As a given dataset may contain multiple different temporal aspects, it was decided to not provide a single timeline to filter the data, but instead to include these within the parallel coordinates. This allows the temporal features to be studied, filtered, and manipulated in the same way as other aspects of the data.

To support exploration and interaction of the complex data, GCPC provides users with multiple controls within the parallel coordinates. Figure 3.3 shows an overview of the parallel coordinates view as displayed inside the GCPC. The name of the dimension and the statistical distribution of the data are shown above each coordinate. The multiple controls displayed under each coordinate allows users to manipulate and interact with this specific dimension.

With high dimensional data, identifying patterns and trends in the parallel coordinates across multiple dimension is not a trivial task. It requires following the data over multiple coordinates and trying to understand the represented visual patterns. Another problem is the difficulty of following the data polylines or trend with qualitative data because they fall into discrete values. These discrete values will have a lot of lines coming into and out of the

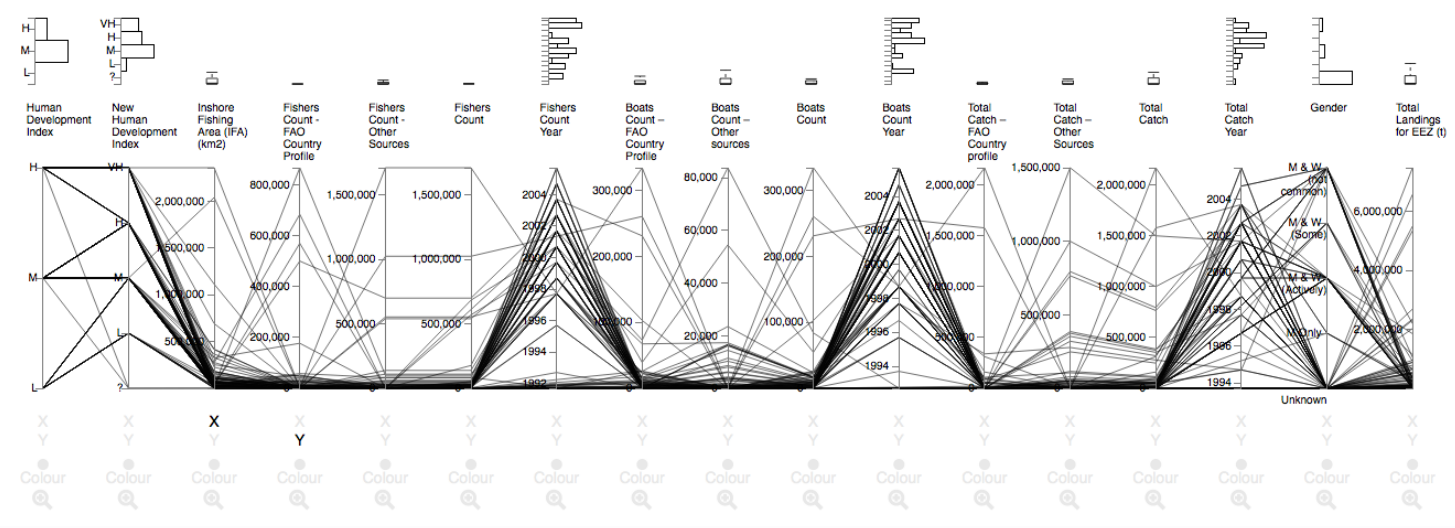

Figure 3.3: An overview of the parallel coordinates view as seen inside the GCPC. 
same location and it will be difficult to follow a specific value across multiple dimensions. To support detecting these trends, the system allows users to encode the colour of the data in parallel coordinates based on a selected dimension.

Users can choose a dimension to colour by clicking on the "colour" control under each coordinate. Figure 3.4 shows the data in the parallel coordinates coloured using the values in the eighth dimension. To enable users to perceive the connection between various aspects of the data, selecting a dimension to encode colour will change the colour used to render the data. The change in colour is reflected in the parallel coordinates and in all the other views (i.e., the geovisualization and investigative scatterplot). The coordination of colour encoding between these views allows users to understand the different aspects of the data and link them together.

In order to ensure proper interpretation of the colour encoding, the encoding scheme is different for each data type. Quantitative data is encoded with a continuous colour scale on a perceptually ordered scale [61, 127]. The perceptually ordered scaled is generated by varying the brightness component of a colour (i.e., empirically selected purple). To encode the data into brightness, the data are normalized from the raw data range to values between $(0-100)$ which are then used as brightness values. The ordinal data (which are discrete in nature) are encoded with a discrete perceptually ordered scale. Similar to quantitative data, ordinal scale vary the brightness component of the colour but at discrete intervals depending on the number of possible values on the ordinal scale.

Encoding qualitative data using the same colour scale will result in interpreting the data as ordered values. To provide a better understanding of such data, qualitative data are encoded with perpetually distinct colour that are easy to distinguish from each other. The distinguishable colours are selected by varying the hue component of the colour and selecting colour on opposite chromatic channels. The number of distinct values in the data is used to divide the hue channel in the HSB colour space and select the colour at equal 


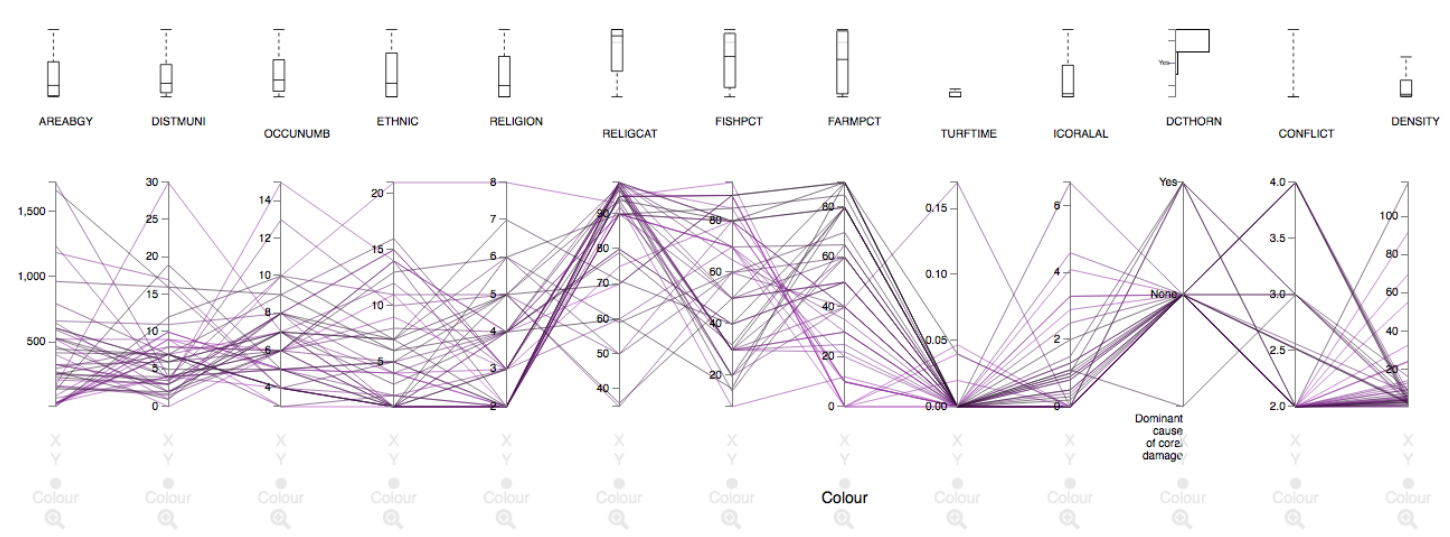

(a) Colour encoding of quantitative data

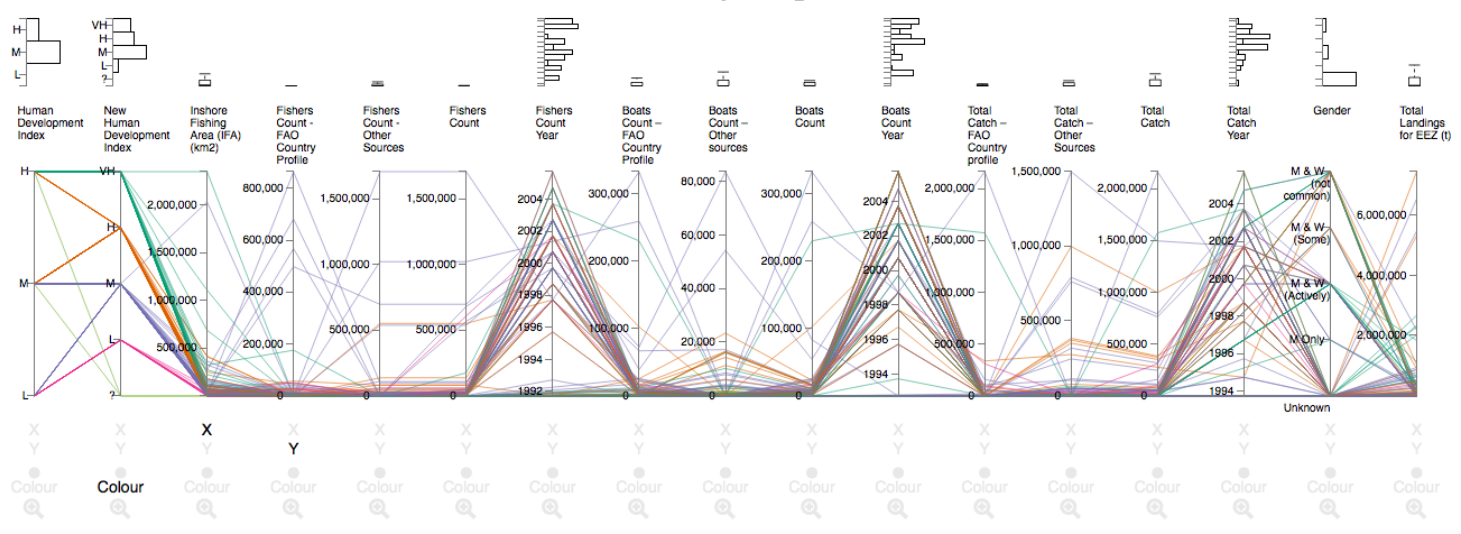

(b) Colour encoding of qualitative data

Figure 3.4: Figure a) shows the colour encoding of quantitative data in the parallel coordinates. In the figure, the eighth dimension is used to encode the colour of data. Figure b) shows the colouring of qualitative data inside parallel coordinates.

intervals from the opposite chromatic channels.

These colour scales were chosen with an awareness of colour theory and the human interpretation of colour [127], using ColorBrewer [24] as the starting point for the specific scale selections. Section 2.1 provides more details about colour selection and Opponent Process Theory of Colour [61]. Figures 3.4a and 3.4b show the parallel coordinates coloured based on quantitative and categorical dimensions respectively.

While there are numerous advantages for using parallel coordinates, there are also a few disadvantages. One of the main disadvantage is over-plotting and the resulting occlusion when rendering a large dataset. Adding interactivity to the parallel coordinates addresses 
part of the problem by allowing users to investigate different configurations and subsets of the data. Filtering and zooming are two of the main interactive features to explore data in any visualization systems. In GCPC, investigating interesting subsets of the data is supported by filtering the data using brushing operations on the coordinates. The user could drag the mouse over any dimension to start the brushing operation, which will filter the data using the selected values. The system supports multiple filtering and brushing at the same time to allow for investigating multiple dimension.

Filtering and selecting data allows users to narrow down the data based on some values. However, if the data is highly skewed or tightly coupled with a small range of values, filtering will not provide users with more details about the data. Detailed analysis of such data is supported in GCPC by allowing users to zoom-in on the brushed data. When users want to zoom-in to the values, they can just click on the "zoom-in" icon under the dimension they want. Zooming-in on the data will re-scale the data displayed in the parallel coordinates based on the selected subset of the data, which will expand the data from a narrow range to the whole range of the parallel coordinates (see Figure 3.5). This allows users to examine a subset of the data that is tightly clustered within a narrow range of values.

Another disadvantage is that due to the nature of the parallel coordinates, detecting direct correlation between the dimensions is limited. While it is easy to identify relations between two successive coordinates, it is hard to recognize direct relations between distant coordinates. This means that the order of the coordinates within the parallel coordinates impacts whether the users can identify relations between dimensions and understand the general trends. The system provides users with the ability to reorder the coordinates to support identifying relations for multiple dimensions. Users may drag a coordinate from one location in the parallel coordinates and drop it in another, which will move the coordinate and all its controls to the new location. The system immediately re-renders the data to reflect the new order of coordinates, allowing users to see direct relations. 


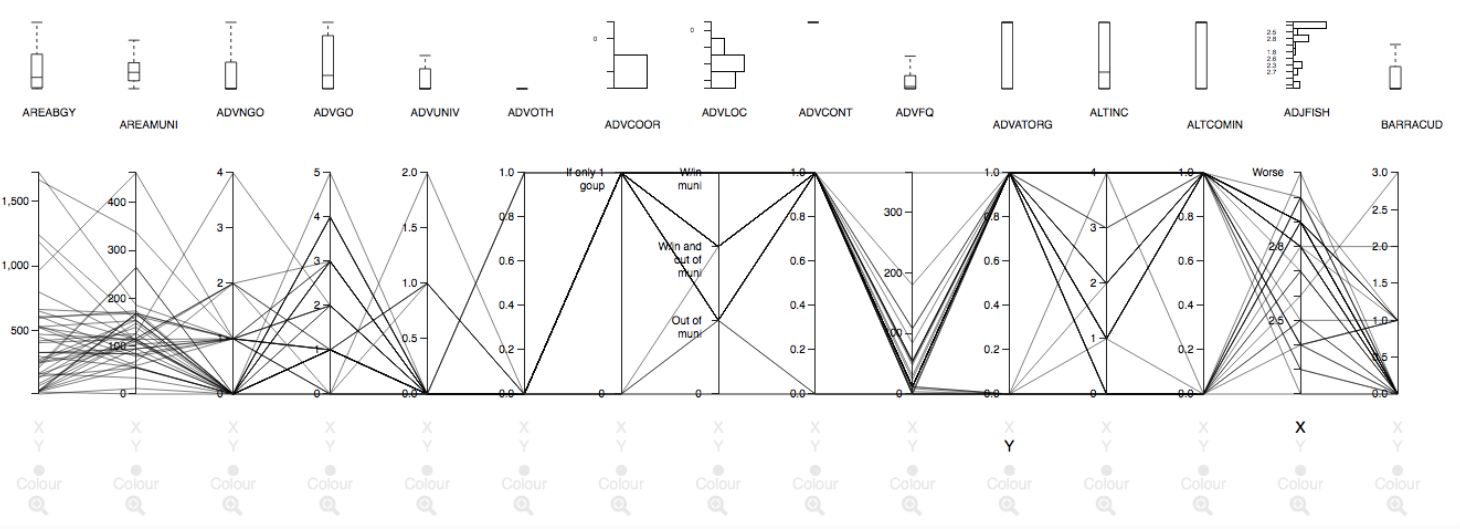

(a) View before zooming in
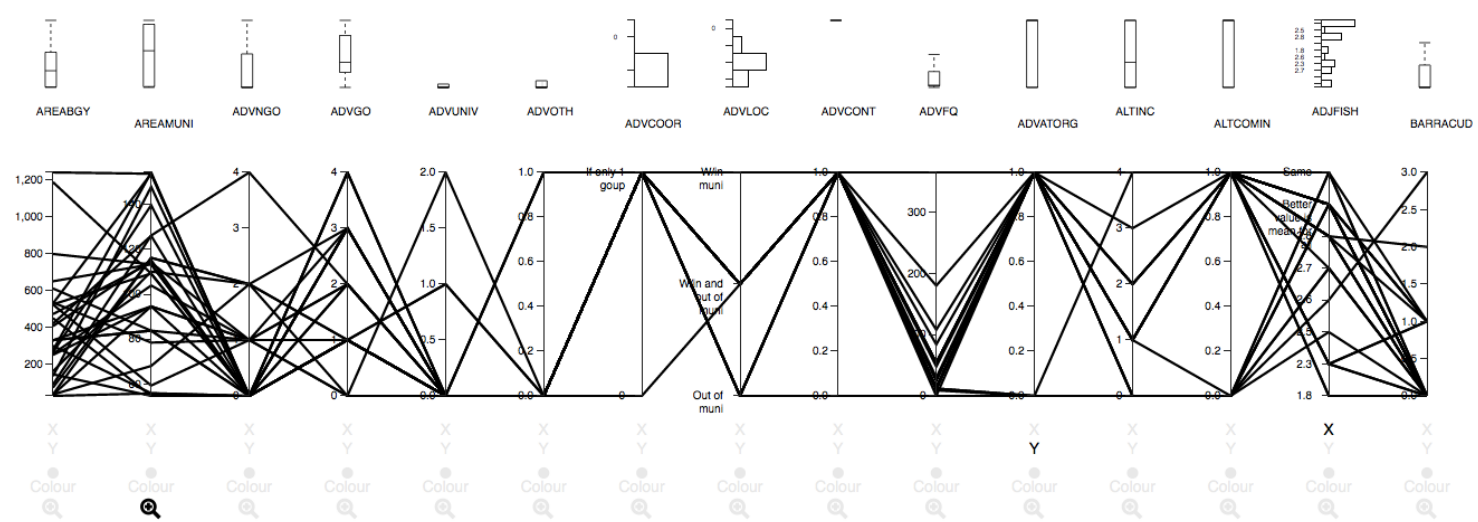

(b) View after zooming in

Figure 3.5: Figure a) shows the view before zooming in. Figure b) shows rescaling of all the dimensions after zooming in to the data.

When the number of dimensions increases tremendously (i.e., more than 50), it becomes extremely difficult to analyze such a large number of dimensions at the same time. To support users in focusing on the most relevant dimensions for their analysis, GCPC allows users to select the dimensions to be included or excluded from the visualization. Based on the system configuration, the initial view of the system could either show the first 15 dimensions in the dataset or a model window allowing users to select which dimension to visualize. During the analysis users may click on the "settings" icon on the left corner of the parallel coordinates to add or remove dimensions from the analysis. The selection dialog contains all the available dimensions in the dataset, permitting users to choose which 
dimensions to add or remove from the analysis. After selecting and saving the dimension to analyze, the system will re-render the parallel coordinates using only the dimensions that have been selected. The set of selected dimensions is saved in GCPC to continue the analysis at a later time.

\subsubsection{Geovisualization}

To allow users to observe and interpret the spatial distribution of the data, a geovisualization is used to visualize geospatial aspect of the data. This is an essential part of GCPC, allowing for the exploration among the relations between multiple factors and the geospatial aspects of the data. Displaying different views of the same data on both the parallel coordinates and the geovisualization, allows users to understand the relations between the different aspects of the data. Detailed analysis and understanding of various relations is supported by tightly coupling the parallel coordinates and the geovisualization using multiple coordinated interactions.

GCPC contains two main modes of displaying geospatial features on the map: a dot map, and an aggregated hexagonal view. The dot map shows the raw spatial point location using circles at the appropriate location. Settings below the parallel coordinate plot allow for the manipulation of two visual variables within the geovisualization: the colour and size of the circle. To coordinate between the multiple views of the system, the colour scale used in the geovisualization is the same as the one used in the parallel coordinates. Depending on the type of data, the scale will either be perceptually ordered or perceptually distinct colours. To encode data to the size (i.e., to the radius of the circles) without adding visual clutter on the map, the data is normalized to values between $(1-10)$.

With large data sets, it is beneficial to have an aggregated overview of the data rather than be distracted by the raw data. The aggregated view allows users to quickly assess the location of a high number of points or grasp the low and high level of distribution of 
the data. GCPC provides overview of the geospatial data using the hexagonal binning that represents aggregated such a spatial data using an hexagonal grid. Figure 3.6 shows an example of both view modes.

While the process for producing the dot map is straightforward, there is some complexity in the creation of the hexagonal bin map. A grid of hexagonal polygons is layered over the map, and the data are aggregated based on which bin it falls into [28, 101]. Based on the raw location of each point of data, the point is aggregated to a specific hexagon. The default is to simply count the number of data points in each bin, but more complex aggregations such as total or average of a given dimension of the data are also possible. The sizes of the hexagons are then used to encode the number of data points aggregated within the bin.

When the data is aggregated in the hexagonal bins, the colour and size encoding cannot be used directly. Instead, the size is used in the binning algorithm to reflect the number of points inside the hexagon and the colour is used to encode the combined value of the aggregated data [28]. The final colour of each hexagon is determined by the average value of quantitative data or the most frequent value of qualitative or ordinal data. As ordinal and temporal data are encoded with a discrete perceptually ordered colour scale, the colour of the hexagons will be determined as in quantitative data by the average of the aggregated

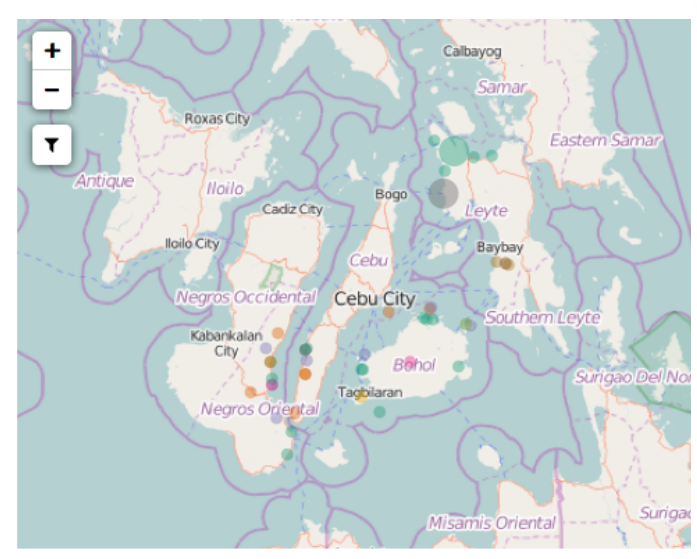

(a) A dot map view

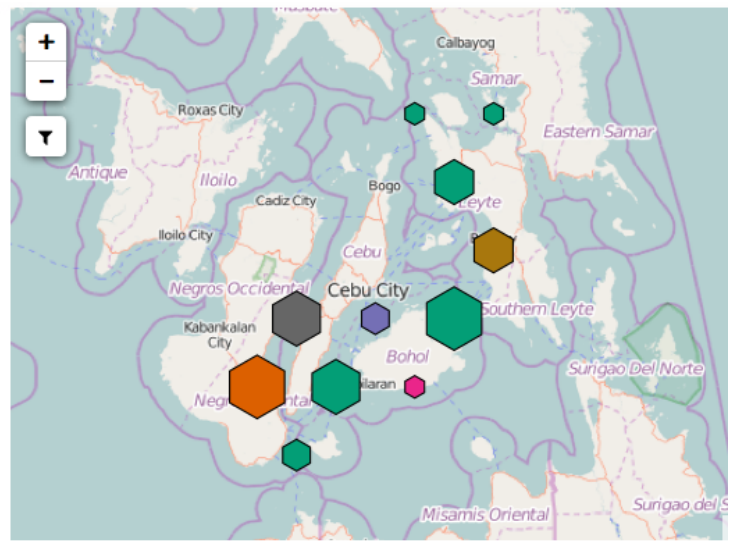

(b) The hexagonal binning view

Figure 3.6: Example of the dot map and the hexbinning views 
data.

The initial view of the data will be zoomed out to give an overview of the whole data extent. The normal pan and zoom operations on the map allow users to view more closely the geospatial relationships among the data. The zoom-in and zoom-out of the hexagonal view will recompute the grid based on the zoomed area, which allows users to get an overview of the data in the zoom out view then get more details when they zoom in.

One of the main coordinated interactions between the parallel coordinates and the geovisualization is the filtering. Any filtering or zooming on a parallel coordinates view automatically filters the data on the geovisualization. The coordination support users to explore and analyze multiple aspects of the data by focusing on a subset of the data in all views. Allowing users to select and filter data based on geospatial location from the map provides them with further exploration capabilities. In order to support the investigative analysis of the data, the geographical filter is automatically coordinated to all the other views. Users can select interesting geographical patterns to focus on and then explore the high dimensional aspect of the filtered data.

Due to the natural irregularity of geographical locations, geospatial data visualized over a map are hard to select and group using regular rectangle filters. For example, selecting data points mapped over Europe without adding extra points could not easily be achieved using regular rectangle selection tools. To allow users accurate selection of interesting geospatial data, the system provides an arbitrary shape geospatial filter. Users can activate the geographical filter and select a geographical location to investigate. The system allows users to draw polygons to create an arbitrary shape that overlaps a region of interest (see Figure 3.7). The filter will remove all data points outside of the drawn shape, both within the geovisualization and also from the other visual representations. However, GCPC will only allow users to draw closed polygons to filter the data.

Another interaction that allows users to link the geospatial data to the high dimensional 

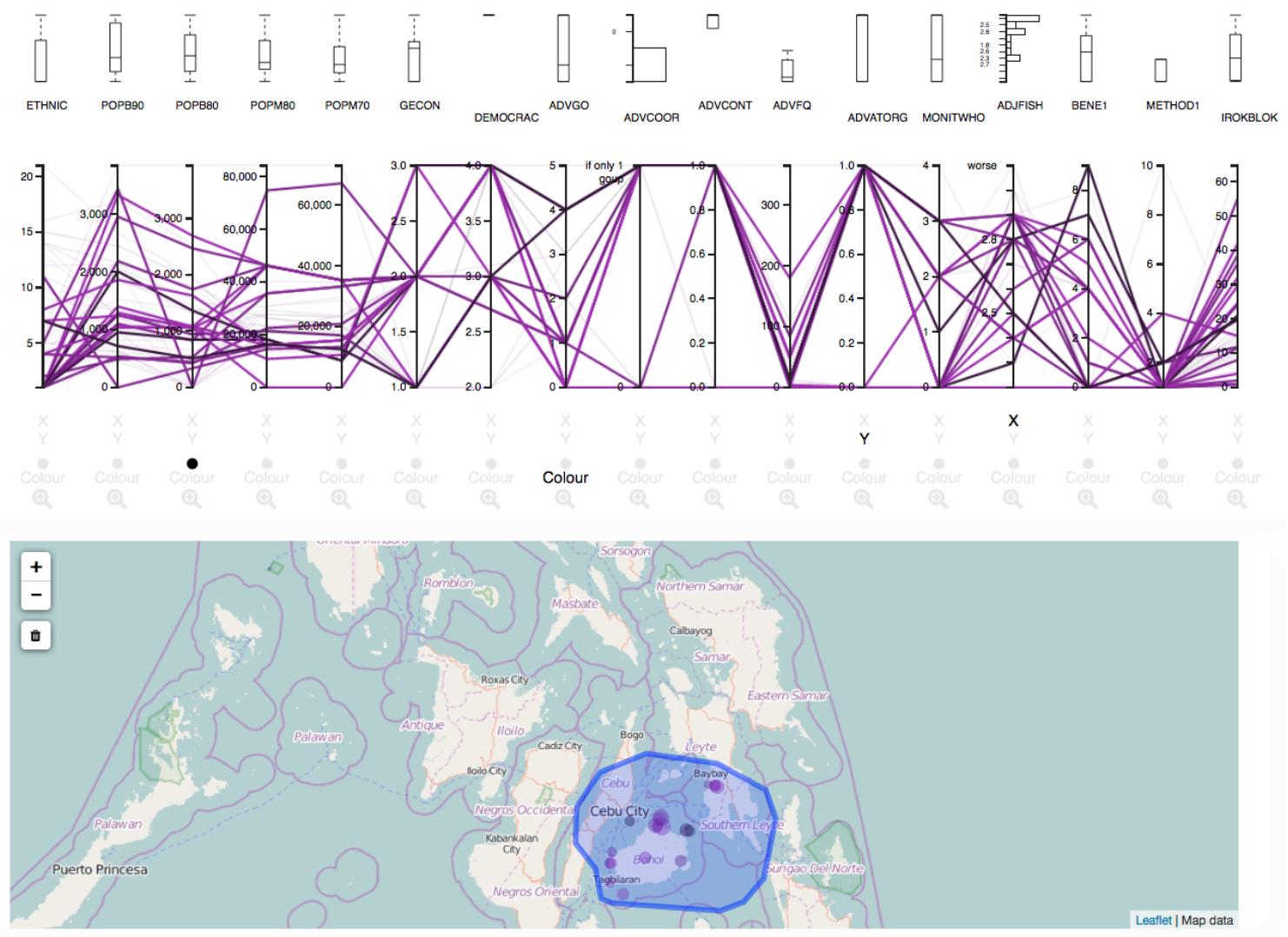

Figure 3.7: Example of using the geospatial filter to filter the data based on arbitrary shape aspect of the data is the coordinated highlighting. The coordinated highlighting allows users to select specific data points within the map in order to highlight their attributes in the other dimensions. When users hover over a point on the map, the point will be highlighted (i.e., visually encoded using a different colour than the rest of the view) in all the other views and the tooltip is displayed. If the hexbining view is active, all the data points aggregated in the hexagon will be highlighted. Additionally, hovering over a point on the map links to the parallel coordinates and vice versa, providing users with quick investigative tools to analyze the relations between the different aspects of the data.

These interactions features enable the co-exploration of the data within both the highdimensional elements and the geospatial elements. Users will be able to relate the different aspects of the data by seeing and interacting with the same data on different visualizations. 
By combing multiple filtering and encoding operations between the geovisualization and parallel coordinates, complex geotemporal patterns and trends can be isolated and analyzed.

\subsubsection{Investigative Scatterplot and Correlation Analysis}

Investigating the correlation between different factors and dimension is essential to understanding the complex relations within the data. While the order of the dimensions in the parallel coordinates may be manipulated to observe the patterns or relationships, analysts may wish to investigate such relationships in more detail and in a more fluid and interactive ways. A scatterplot is a powerful technique used to investigate direct and indirect relations between different dimensions of the data [73].

A scatterplot is a 2D visualization with horizontal and vertical axes to represent two dimensions. Datapoints are represented by points scattered in the $2 \mathrm{D}$ space with respect to their values in each dimension. Using the scatterplot, the clusters, trends, and direct correlations of the two dimensions are easily identifiable [73, 126]. Traditionally the scatterplot visualizes two dimensional data using orthogonal axes, but more dimensions could be encoded in the graphical properties of the points. Using colour and size to encode other dimensions of the data in the scatterplot provides users with a means to identify indirect relations between the encoded dimensions. By allowing users to select which dimension to encode in the scatterplot, the visualization provides a tool that support detailed investigations of the different correlations between multiple dimensions.

In order to support such exploration of the possible relations between various dimensions, GCPC allows users to select and visualize up to four dimensions at the same time. Users may start their analysis by choosing the two dimensions of the data to plot using the controls under the parallel coordinates. Doing so results in the creation of a scatterplot of the data. By selecting to encode specific dimension on the $\mathrm{x}$ - and $\mathrm{y}$ - axes, the scatterplot enables a direct and intuitive analysis of the correlation between these selected attributes. 
The system also allows users to select which dimension to encode as colour, size, or both, allowing for the interactive visualization of four dimensions of the data. In order to easily coordinate between the multiple views in GCPC, both the colour and size scale are the same throughout the system. As in the parallel coordinates and the geovisualization, the colour scale is based on the type of data. As users can easily change which dimension are displayed in the scatterplot, correlations can be investigated quickly and easily.

Figure 3.8 shows how users could interact and use the investigative scatterplot. Figure 3.8a shows using the scatterplot being used to visualize the "Area" and the "Population" of barangay (i.e., a village). A number of interesting clusters and patterns can be easily identified from the scatterplot. Adding another dimension to the visualization may provide more understanding of the underlying data. For example, users may then add another dimension either by encoding it as colour (see Figure 3.8b) or as size (see Figure 3.8c). Figure 3.8b shows that by encoding "Conflict" to colour, it is clear that there is more conflict in regions with low population and smaller areas than in larger regions. Looking at Figure 3.8c, users could recognize the relation between "Population" and "Employment rate". Using both colour and size, the scatterplot could be used to confirm or deny any relation between these dimensions (see Figure 3.8d). By allowing users to dynamically select the dimensions, users are able to investigate the different relations in the data. Permitting users to examine and confirm the multiple possible relations within the data will help them to identify the various patterns and trends in the data.

Following the same coordinated interaction within the other views of the data, the scatterplot provides multiple interactions that are automatically coordinated to the other views. Users may use the scatterplot to filter data based on specific clusters or regions in the scatterplot. A rectangular filter is utilized by brushing over a region of the interest in the scatterplot. The brushed points are highlighted in the scatterplot view and filtered from the other views. The user activates the brush by clicking on the "crop" icon over the scatterplot, 


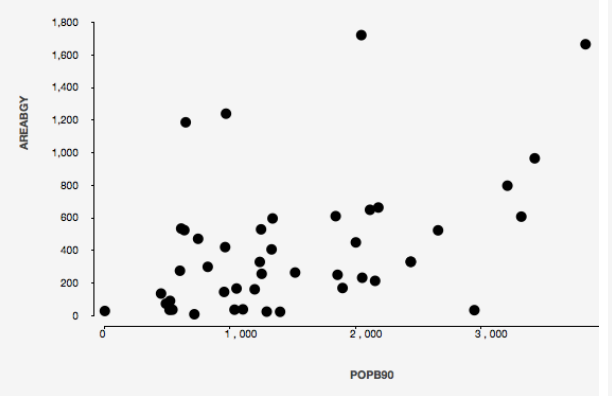

(a) Scatterplot encoding 2D

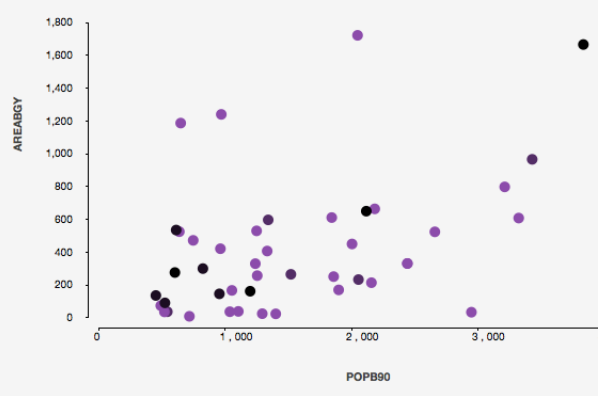

(b) Scatterplot encoding 3D

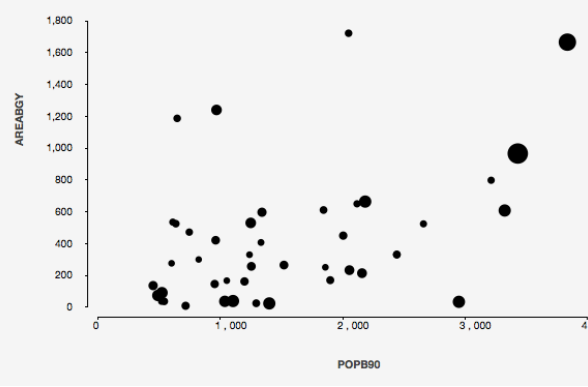

(c) Scatterplot encoding 3D

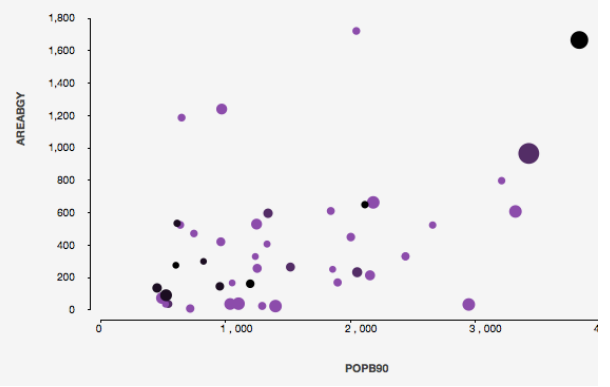

(d) Scatterplot encoding 4D

Figure 3.8: Example of using the investigative scatterplot (a) Shows the encoding of 2 dimensions (i.e., area of and population of barangay) to the x-axis and y-axis. (b) Shows the encoding of 3 dimensions (i.e., the area of barangay, the population of barangay, and conflict) to x-axis, y-axis, and colour. (c) Shows the encoding of 3 dimensions i.e., the area of barangay, the population of barangay, and employment rate) to $\mathrm{x}$-axis, y-axis, and size. (d) Shows the encoding of 4 dimensions (i.e., the area of barangay, the population of barangay, conflict, and employment rate) to $\mathrm{x}$-axis, $\mathrm{y}$-axis, colour, and size.

then brushes over the region inside the scatterplot to filter the data. All points outside this rectangle region are removed from all the other visualizations. The user can remove the filter by clicking on the "crop" icon for the second time. Selecting individual points will highlight their counterparts within the parallel coordinates and the geovisualization.

Another interaction supported in the scatterplot is highlighting of point when users hover over a point in the plot. Hovering over a point on the scatterplot will show the tooltip on the scatterplot and highlight the data point in both the parallel coordinates and the geovisualizaiton. This allows users to connect and relate the point in the scatterplot to the other views, which supports users in exploring and investigating the different aspects 
of the data that are not shown in the investigative scatterplot.

\subsubsection{Statistical Descriptors}

One main criticism of using parallel coordinates is the difficulty in identifying the distribution of the data on a given dimension. The problem is more evident with large datasets where a large number of lines are drawn in the parallel coordinates. The compact nature of the parallel coordinates may result in overplotting and visual clutter, making it difficult to identify the precise number of data points going through a given value on a specific dimension. As a consequence, it is hard to spot frequent values for specific dimensions or recognize other statistical properties of the data.

To address this limitation, micro-visualizations have been added to each parallel coordinate to illustrate the statistical properties of the data. While others have explored sim-

ilar solutions by overlaying the statistical descriptors on the coordinates themselves [62], GCPC provides these on top of each coordinate, allowing the information to be observed as needed without interfering with the interpretation of the data shown in parallel coordinate plots.

Depending on the data type, the important statistical information is different. Quantitative data could be described using average, standard deviation, and similar statistical descriptors. On the other hand, qualitative data are better described by the frequency of occurrence in the data. To support analysis of the different types of data, the format of these statistical descriptors depends on the type of data they describe.

Tukey box plots [120] are a common visual encoding that provides a compact representation of the distribution of a set of numerical values. Quantitative data is visualized using the Tukey box as it represents a convenient method to display statistical properties about a set of data. It makes it easy to observe distribution properties which includes: average, max, median and quartiles information. Figure 3.9 illustrate how to read a box plot and 


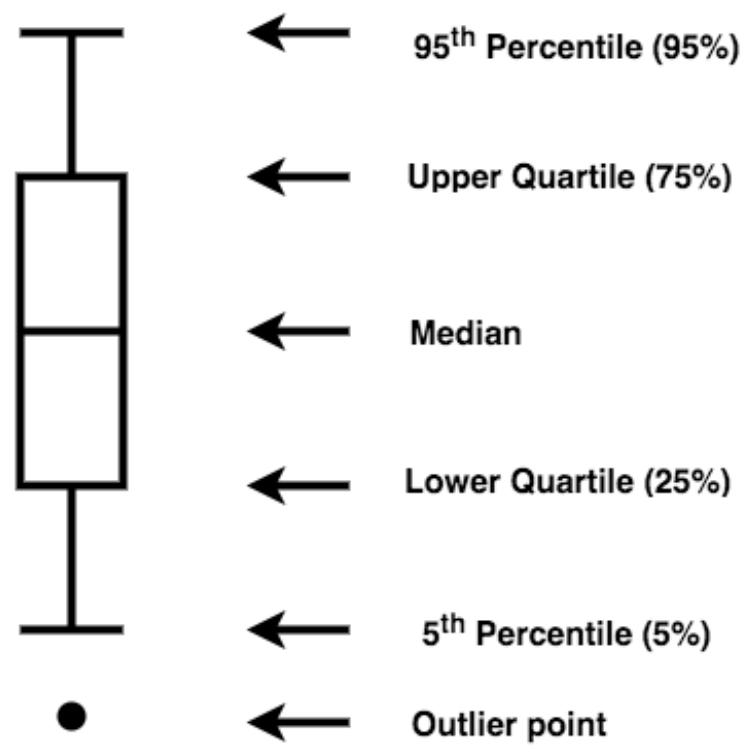

Figure 3.9: Example of a Tukey box plot

where to find the different quartile information. The box boundaries represent the lower 1/4 and upper $3 / 4$ quartile of the data. The median is displayed by a horizontal line inside the box of the plot. The whiskers show the fifth and ninety-fifth percentile of the data. Data outside the fifth and ninety-fifth percentile are shown as points.

Using the plot for multiple dimensions makes it easy to estimate the spread of the data and allows users to compare its distribution. It is a concise way to compare distributions as it allows observing if the data is symmetrical, if there is a skew in the distribution, or how tightly the data are grouped. Figure 3.10 shows an example of the box plot across multiple dimensions. It is easy to identify the dimension where points are distributed evenly, the dimension with large spread over the values, or the dimension tightly grouped in lower values.

For qualitative and ordinal data, measuring the quartile and median is meaningless; therefore another graphical representation is needed. A histogram of the distributions is used instead, to allow users to observe the frequency of each category. A micro-histogram is represented using a bar chart to visualize the frequency of specific values inside the data. 


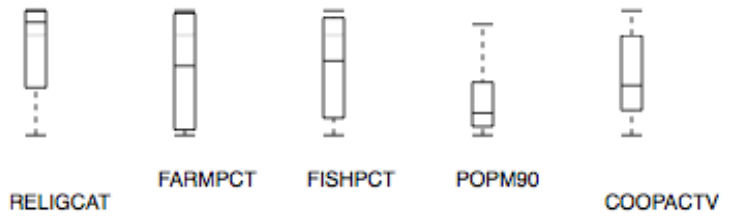

Figure 3.10: Tukey box plot for multiple dimensions

Figure 3.11 show an example of the histogram plot over the dimensions of three parallel coordinates.

While the initial computation of the statistical information is important to provide an overview of the distribution of the data, any filtering or zooming on the data from any view will change the values displayed and along with it the distribution of the data. Recalculating and rendering the statistical properties whenever users filter the data provides the users upto-date statistical properties of the filtered data. The changes between the distributions of different subsets of the data allows users to investigate various relationships and patterns in the filtered data.

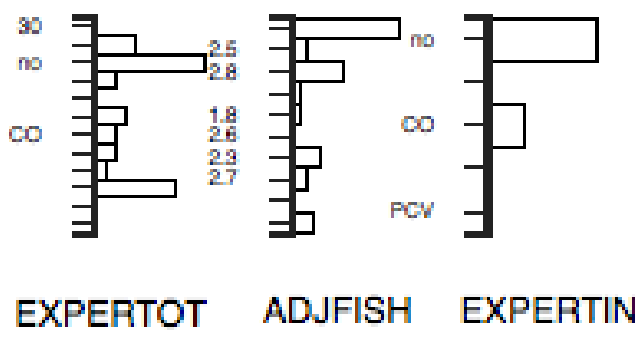

Figure 3.11: Example of histogram plot for multiple dimensions 


\subsubsection{Outlier Detection}

An outlier is defined as a data point that is notably different from the rest of the dataset [3]. Algorithms that detect outliers generally create a model of the normal patterns and data distribution, which they use to label data points that do not fit the model as outliers [3]. Outliers are important as they may represent noisy data, significant abnormal incidence, or erroneous data points.

One of the criticisms of parallel coordinates is the difficulty in identifying outliers, due to the significant visual weight that is given to the dominant pattern within the data [60, 96]. As a result, it is difficult to visually isolate data points that are different from the norm. In some cases, such outliers may be uninteresting, and there may be a desire to remove these to reduce the additional visual clutter they cause. In other cases, the outliers may be important for the analysis at hand, and there may be a desire to highlight them. In order to support outlier analysis, GCPC includes an automatic outlier detection algorithm. The algorithm detects the outliers in a given dataset and then allows users to manipulate them.

A variety of methods have been developed to detect outliers in a given dataset. Extreme values methods are simple techniques where the normal probability distribution of a dataset is computed and an outlier is detected if it lies in the extreme ends of the normal distribution [3]. Cluster based methods group points to multiple clusters and considers a point without a cluster an outlier [3]. Distance based models try to compute the distance of each point to the nearest $k$ clusters or distribution. If the distance is larger than a pre-set threshold then the point is considered an outlier [26].

While there have been multiple algorithms to detect outliers, most of these algorithms lose accuracy when they try to model high dimensional data [3]. Extreme values are harder to detect in high dimensional data even if the algorithm uses probability distribution to model the extreme values. Clustering based method will rely on the ability of the clustering algorithm to group high dimensional points. Few outlier algorithms adapt to high 
dimensional data effectively [81, 109, 26].

GCPC employs an outlier detection algorithm that is designed specifically for high dimensional data. Angle based outlier detection a (ABOD) is based on the comparison of the angle between high dimensional vectors [81]. This approach is useful as it reduces the effect of the curse of dimensionality by using the angles between vectors instead of other distance measures. A second advantage of this method is it does not require prior knowledge of the data distribution or training on a sample dataset to detect outliers.

The algorithm computes an outlier score based on the variance of the angle between pairs of high dimensional vectors [81]. The algorithm is based on the observation that a point inside a cluster will be surrounded by other points. This causes the angle between the vectors from this point to other pairwise points to be wider than a point outside the cluster. As the point will be surrounded by multiple points in all directions, the angle between it and all other pairwise points will have a wide range of values. On the other hand, in the case of a point outside a cluster, the point will be farther and the angle of pairwise points will be smaller. As the outlier point will be further away from the other points, this angle between vectors will have small variance with respect to other points.

Figure 3.12 illustrates the observation where angle $\gamma$ (i.e., from the outlier point) is smaller than angle $\beta$ (i.e., from the point on the border of the distribution), and both are smaller than angle $\alpha$ (i.e., from a point inside the distribution). A closer look at the outlier point in the Figure shows that the variance of angles between the outlier point and all other pairwise points will be small relative to the other points.

Prior to computing the outlier score, the angle between two vectors from a point $A$ in the dataset to all pairwise points (i.e., points $B, C$ ) is computed. The statistical variance of the computed angle for each point in the dataset indicates the location of the point in the distribution. This variance is computed for every point in the dataset and used as outlier score. The points that are inside the distribution will have high variance as they will have 
large differences in the angles to other points. In contrast, outlier points will have a smaller variance indicating the low difference in angles between different pairwise points.

The angle based outlier detection $A B O D$ score of point $A$ is computed as follows:

$$
A B O D(A)=V A R_{A,(B, C) \epsilon D}\left(\frac{\langle\overline{A B}, \overline{A C}\rangle}{\|\overline{A B}\|^{2}\|\overline{A C}\|^{2}}\right)
$$

Where $A, B$, and $C$ represent data points in the dataset $D$, and $V A R$ is the variance of point $A .\langle\overline{A B}, \overline{A C}\rangle$ is the angle of the high dimensional vectors from point $A$ to points $B$ and $C$ (i.e, $\overline{A B}$ and $\overline{A C}$ ). $V A R$ is the statistical variance of the angles between point $A$ and all pairwise $B$ and $C$ in the dataset $D$. The length of vectors $\|\overline{A B}\|,\|\overline{A C}\|$ are used to normalize the computed angle, which gives more weight to the score if points $B$ and $C$ are nearer to point $A$. The $A B O D(A)$ is computed for every point $A$ in the dataset $D$.

Once the variance is computed for each point, the system ranks the points based on this computed score and use it as the outlier score. Following this ranking, the points are labelled as outlier if the score is lower than an empirically set threshold (i.e., 5\% of the maximum score in the dataset).

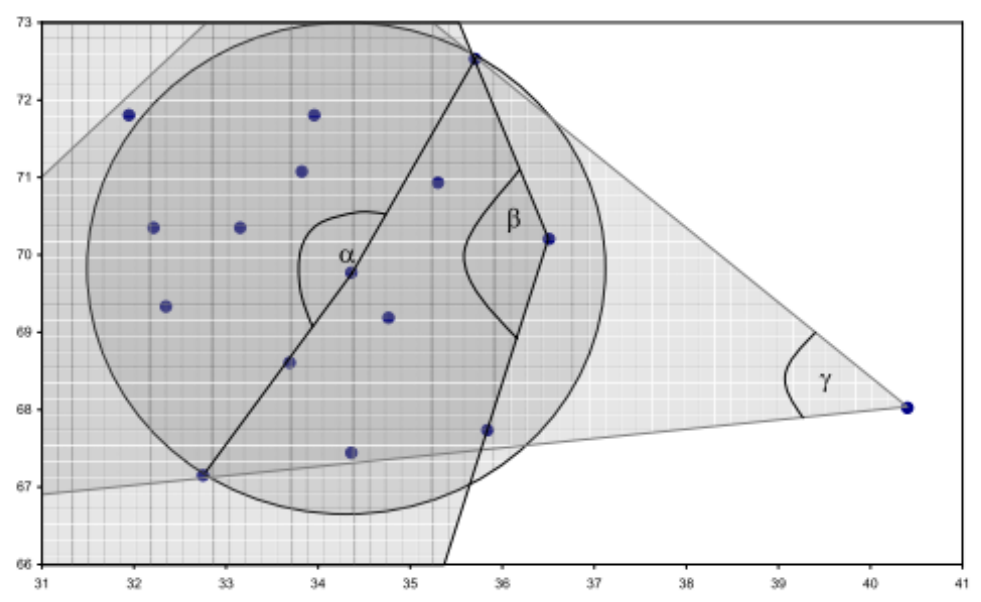

Figure 3.12: The observation behind angle based outlier detection algorithm [81] 
As the system supports high dimensional heterogeneous data, mapping the different types of data to numerical values is required before running the algorithm. The algorithm computes the angle between high dimensional data, but for accurate computation of the angle the values need to be numerical. Although not entirely accurate, the system normalizes qualitative and ordinal data and maps them to numerical values between $(1-100)$. This allows the algorithm to compute the angle between different qualitative values, but these angles may not entirely accurate.

Because this algorithm must compare each data point to all pairwise points, it is computationally expensive $\left(O\left(n^{3}\right)\right)$. While classifying the data using an algorithm such as k-nearest neighbors can speed up the approach [81], for our purposes, it is not necessary to calculate these outliers in realtime. Instead, the outlier scores and ranking can be calculated during the preprocessing step and stored as part of the data, but keeping the cutoff threshold for outlier classification as an interactive parameter.

GCPC allows users to explore the outliers in multiple ways. Users may start the outlier analysis by clicking on the outlier button. Users may then choose whether to use this to filter out the outliers or highlight them for detailed investigation. Filtering the outliers will remove them from the current view in the parallel coordinates. Highlighting the outliers will select them in the view and allow users to further explore them using GCPC. This allows users to investigate the details of the outliers and check if they are actually interesting before either removing them from the analysis or keeping them as they are.

Users may then choose how sensitive to make the outlier detection, a slider is provided so users can change the cut off threshold of the outlier algorithm. The slider controls the threshold that labels the outliers based on the ranking of the ABOD algorithm. Users can move the slider to change the threshold and see the change in the selected outliers view, or remove additional outliers from the view. 


\subsubsection{Data Inspection}

During the exploration of the data, it is important to maintain the ability to drill down to the raw data in order to allow users to inspect the actual values. This inspection may be used by users to confirm what has been shown visually. The details should provide users with ability to see the context of the data and investigate the visual representation of the data.

The system provides users with various methods to inspect and view the raw data. The first is the tooltip that appears when users hover over a single point either on the map or the scatterplot. Hovering and selecting a single line from the parallel coordinates using a mouse is difficult and error prone. Therefore, we did not attach a tooltip to the parallel coordinates lines. The tooltip displays the value of the location and any dimension selected (i.e., from the parallel coordinates controls) to encode a visual parameter. Selecting and clicking an individual point in either the map and scatterplot views will highlight the point in all the views and show the details window. The system populates the details window with the complete set of data from this point to provide all the information about the specific point. Figure 3.13 shows a section of the inspection window, which displays a table with the complete data.

The second data inspection tool available to users is the detail table of the dataset. The

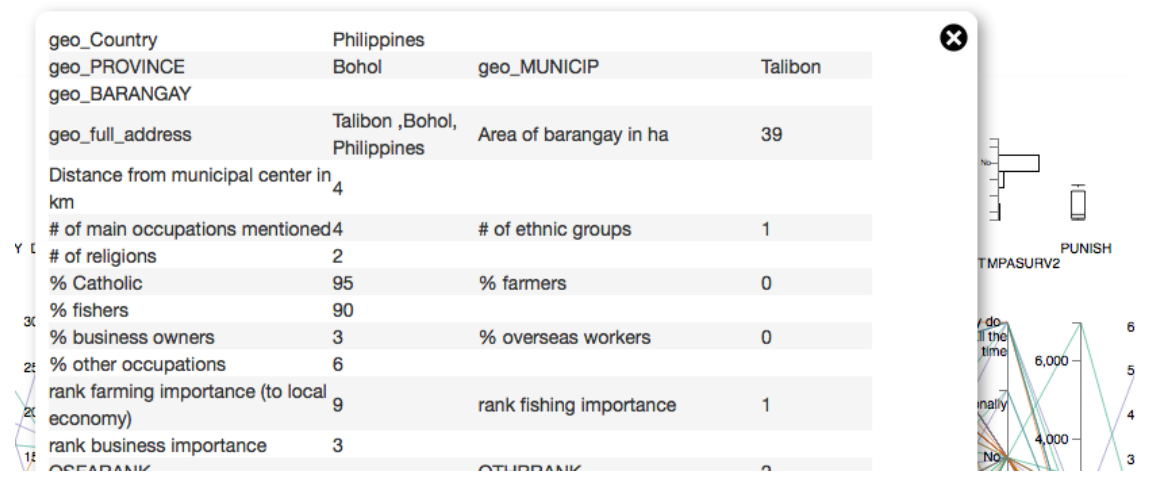

Figure 3.13: Inspection window 
table permits users to display information in a tabular format which could be easily exported and copied to other systems. The table format enables users to get the exact values of every point in the data that is currently being shown (i.e., based on filtering operations). To adhere with our goal of displaying details only on demand, users could show or hide the detail table.

To support the exporting of the results of the analysis to another system, the table view is coordinated with all the other views. The table will only show the details of the subset of the data that are displayed in the other views. Any filtering of the data in the parallel coordinates plot, map, or/and scatterplot will be reflected in the table view. Highlighting and selecting points are also coordinated with the table view. In all the views, when users hover or select a point the respective row in the table will be highlighted.

\subsection{Exploration Scenario}

This section describes an exploration scenario of a dataset from the fisheries domain. The dataset was provided by the Too Big To Ignore (TBTI) research project, whose goal is to document and study the impact and importance of small scale fisheries around the world [51]. It consists of 127 data points over nineteen dimensions that include quantitative, qualitative, ordinal, temporal, and geospatial attributes that describe the small-scale fishing industry around the world.

Before displaying the visualization, GCPC processes the data to organize it in the appropriate format and compute the initial statistical information. The initial view of the system allows for an overview of the data, which will show the important high dimensional and geotemporal aspects of the data (see Figure 3.14a). One aspect of the data that can be readily observed from the overview is that it is highly irregular, with a small number of extreme values that extend the range of some coordinates (e.g., Inshore Fishing Area (third 
parameter), and Fishers Count (sixth parameter)).

The system permits users to filter, zoom, and explore the data in multiple ways. Using the built in mechanisms in GCPC, the interactive focusing, filtering, and zooming features on the coordinates of interest can address this problem. To focus on the data in the Inshore Fishing Area dimension, the researcher needs to drag a bounding box over the coordinates to filter the data on this dimension. Clicking on the zoom icon will cause the selected range to fill the available space for the coordinate in question. To focus on countries with a low number of fisheries, the researcher can filter the lower part of the Fishers Count coordinate. The results of filtering and zooming operations can be seen in Figure $3.14 \mathrm{~b}$

To further analyze the data, the researcher may be interested in the development and testing of an hypothesis that relates Total Catch, Boats Count, Fishers Count, and Total Landings. A first step in such an examination is to re-order the coordinates such that they are adjacent to one another (see Figure 3.15a). More complex relationships can be observed using the investigative scatterplot, mapping these attributes to the $\mathrm{x}$-axis, $\mathrm{y}$-axis, colour, and size options. (see Figure 3.15b). Since the colour and size parameters are also represented on the map, the locations where the Total Landings and Fishers Count are large can be observed (see Figure 3.15b).

After the previous observation and exploration, suppose the researcher wishes to study the gender distribution of small-scale fishers between Europe and Africa. The map can be zoomed to these regions independently, and then free-form shapes can be drawn around the areas of interest. Doing so filters the data shown in the parallel coordinates, which may be further filtered, perhaps in order to focus on the gender distribution in the most recent data (Figure 3.16a and 3.16b). 


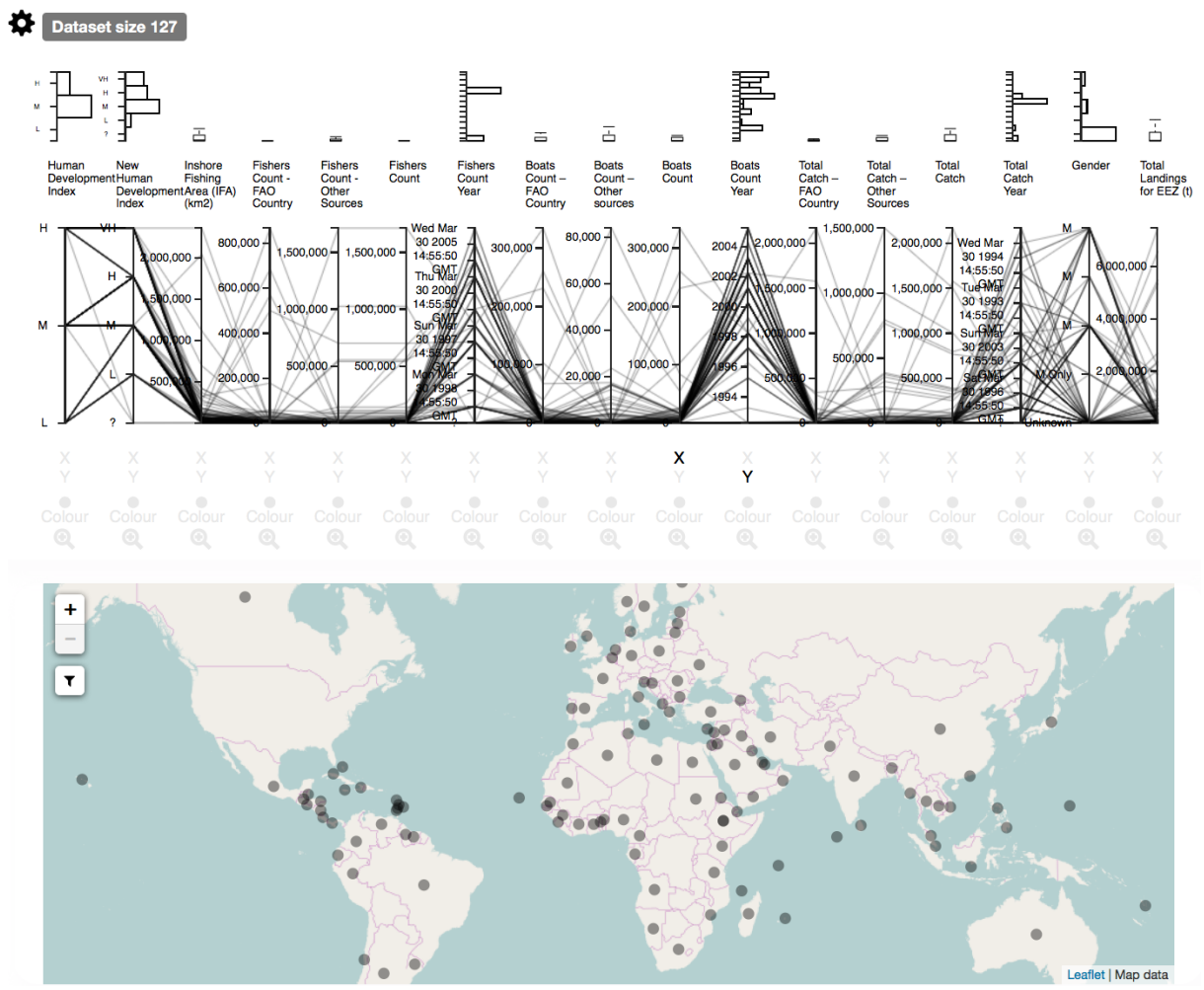

(a) First view of the system

Dataset size 114

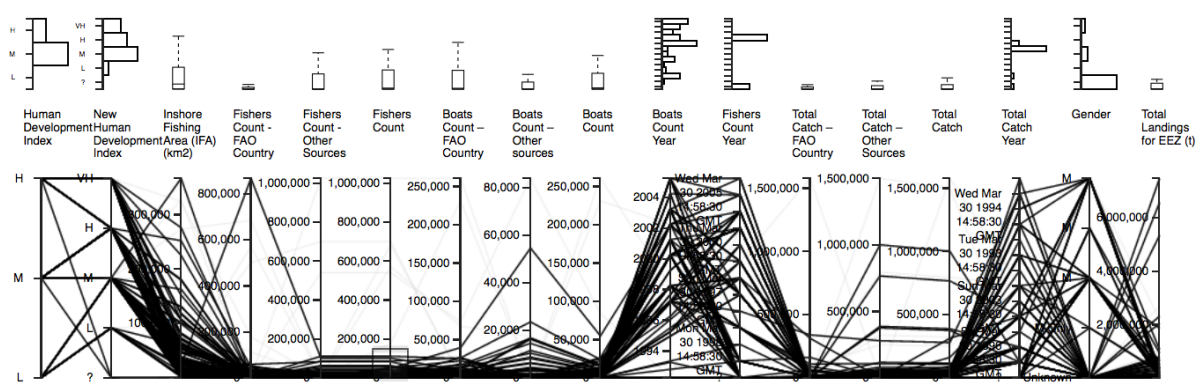

Q

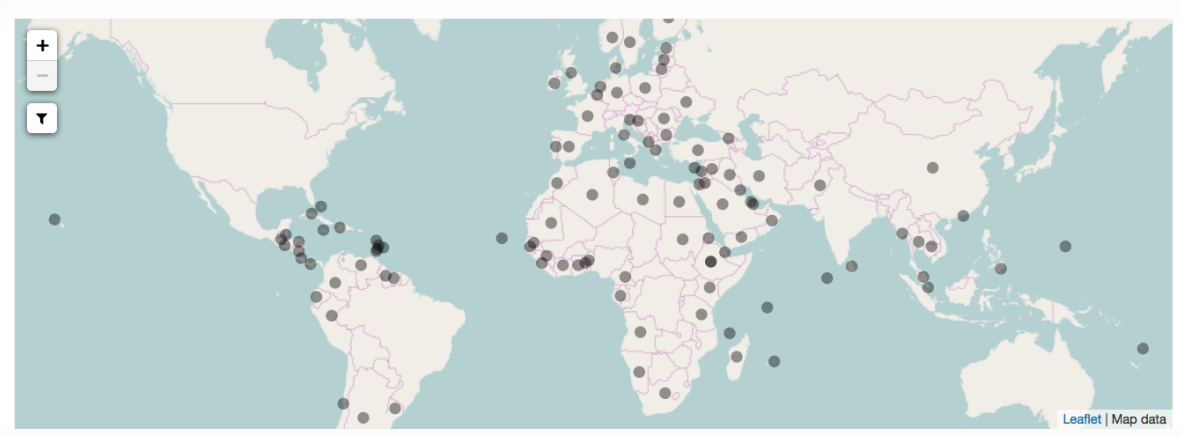

(b) Zooming in to main values

Figure 3.14: Exploration scenario: overview and zoom 76 


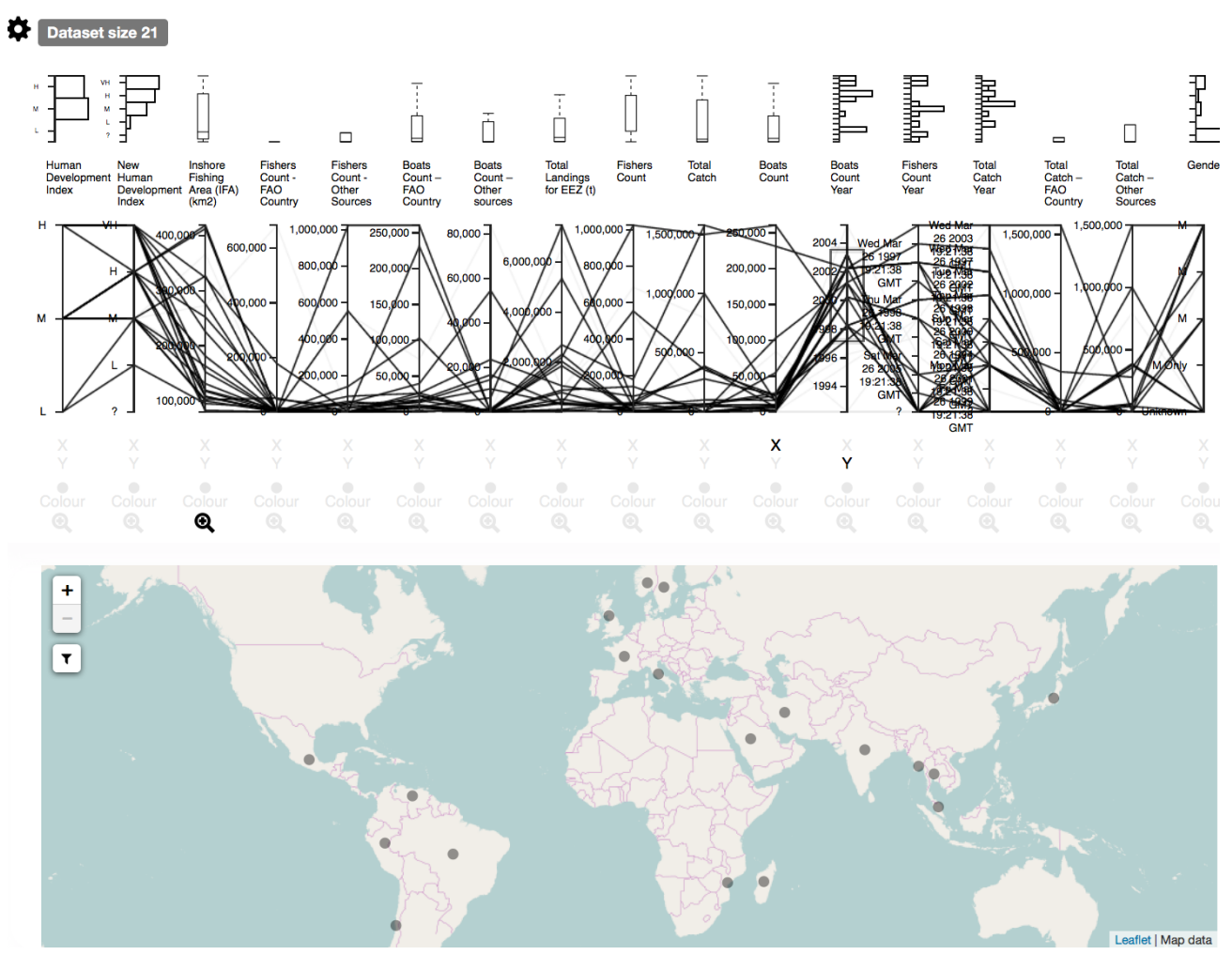

(a) Reoerdering the dimensions
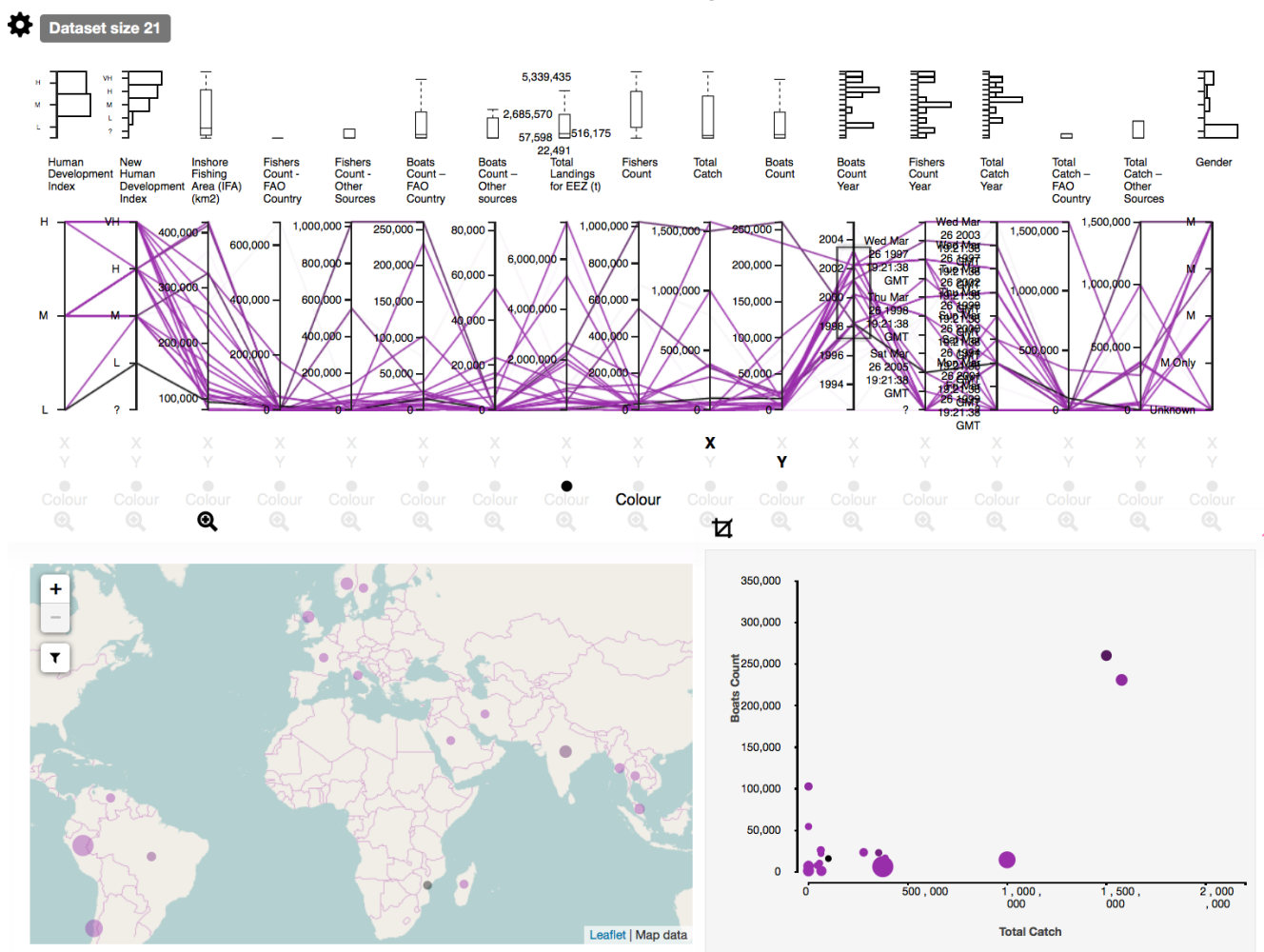

(b) Using the investigative scatterplot

Figure 3.15: Exploration scenario: furthur analysis 

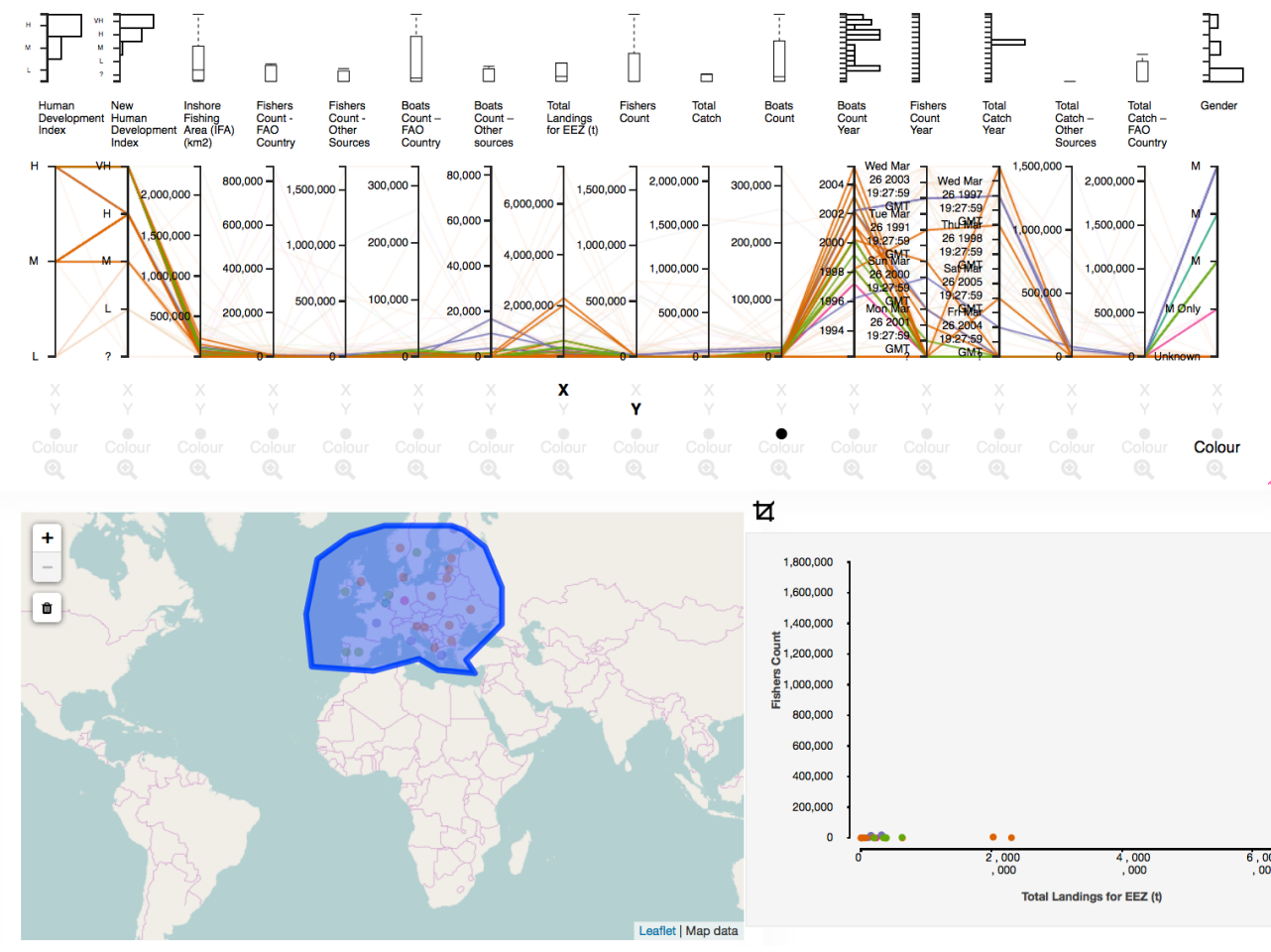

$\square$

(a) Geofilter on Europe

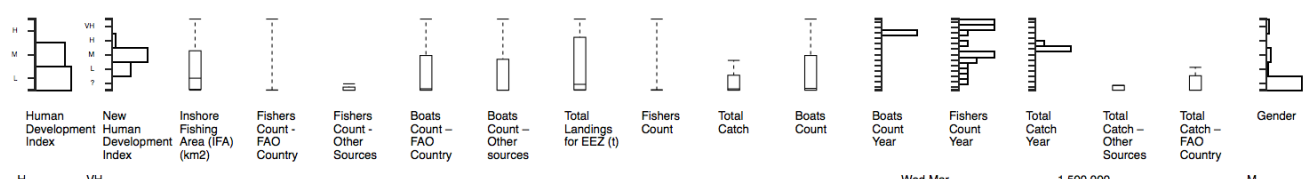
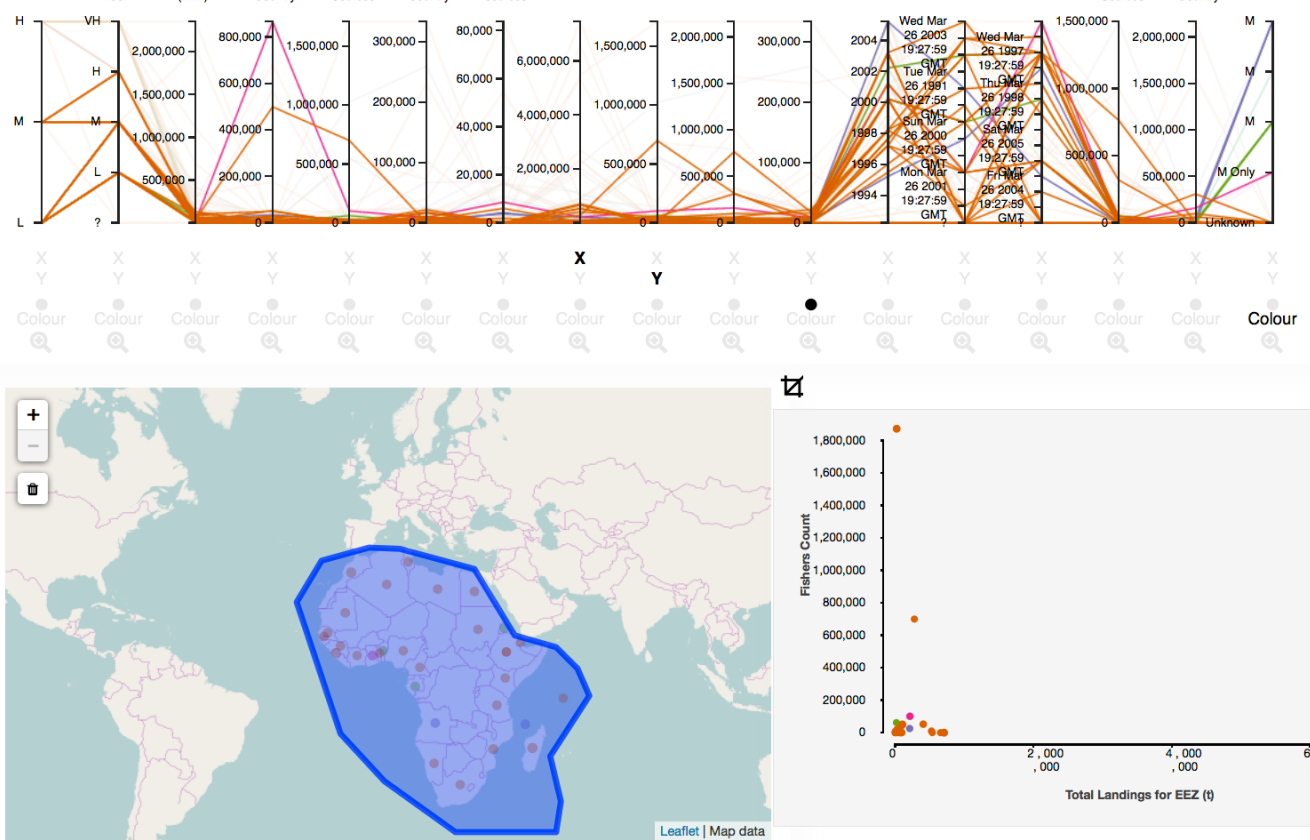

ॠ

(b) Geofilter on Africa

Figure 3.16: Exploration scenario: exploring using the geofilter 


\subsection{Discussion}

Understanding high dimensional data that has multiple aspects is a complex task that requires linking and analyzing the relationships between these aspects. While analyzing heterogeneous high dimensional data is a complex task, adding geotemporal aspects of the data is even more challenging. Analyzing and understanding the wide range of diverse dimensions or factors and their complex relations with the geotemporal aspect of the data is an ongoing research problem. This work addresses challenges in analyzing high dimensional heterogeneous geotemporal data. The research focuses on problems in exploring and analyzing environmental datasets, as an example of geotemporal data with a mix of quantitative, qualitative, ordinal, and temporal data.

This work presents Geo-Coordinated Parallel Coordinates, a system to support the analysis of complex environmental data. The design and implementation of GCPC address challenges of high dimensional heterogeneous geotemporal data analysis by adopting a visual analytics approach. By combining information visualization, data processing, and interactive interfaces, users are able to make sense of the highly complex data [75]. The design of the system adheres to a set of design goals, which focused on supporting visual exploration of high dimensional heterogeneous geotemporal data with multiple coordinated interaction, flexibility with respect to supporting a board range of datasets, and easy communication for the gained insights.

GCPC employes multiple visualizations to depict the various aspects of the complex data. Tightly coupled parallel coordinates and geovisualization allow users to view the high dimensional heterogeneous and geospatial aspects of the data at the same time. Additionally, an investigative scatterplot is used to support interactive analysis of correlations between user specified multiple dimensions. The tight coupling between all the views of the system allows users to relate and understand the different aspects of the data. 
Additionally, to support the analysis of different types of data, the techniques for handling each type are different throughout the system. For example, to ensure proper interpretation of colour, the colour encoding scheme is different for each type of data (e.g., perceptually orders colours for ordered data). Additionally, the statistical properties are displayed using different visual encoding for each discrete or contentious data. The different micro-visualization formats allow the analyst to quickly observe and interpret the different types of data, providing an overview of the features of the dataset.

The coordination between these views allow users to explore different subsets and aspects of the data. While navigation and filtering allows users to explore and focus on subsets of the data, allowing users to change how the data is visualized also supports the understanding of patterns and features in the data. Using multiple interaction and combining the different filters provides users with powerful analysis capabilities, allowing them to understand the complex patterns and trends in the geotemporal data.

Engaging the user in the analysis process is a fundamental requirement of any exploratory data analysis approach. GCPC is designed to support users in their knowledge discovery through the visual analytics process. The system follows Keim's visual analytics mantra "analyze first; show the important; zoom, filter, and analyze further; details on demand" [75], as previously discussion in Section 2.2.

Before displaying the visualization, GCPC processes the data to organize it in the appropriate format and compute the initial statistical information. The initial view of the system allows for an overview of the data previously preprocessed data, which will show the important high dimensional and geotemporal aspect of the data. The system permits users to filter, zoom and explore the data in multiple ways. These interactions and manipulation of the data are reflected in all views to provide users with a deeper understanding of the different aspect of the data By combining these multiple interactions in the system, users are able to explore the different subsets of the data and investigate their hypotheses 
in more detail.

Users will be able to further analyze the data using their interaction with the system and using the multiple automated analysis methods provided by the system. Interacting with the investigative scatterplot allow users to investigate the correlation between the different dimensions. Additionally, the outlier detection algorithm will permit users to automatically detect outliers points and examine if they are beneficial to their analysis. There are various methods that the system uses to provide users with details of the data, allowing them quick and easy access to details on demand. 


\section{Chapter 4}

\section{Field Trial Evaluations}

\subsection{Introduction}

An integral part of research is evaluating the work to ensure that the proposed solution addresses the main challenges, and to assess the benefits of the proposed approach. In information visualization and visual analytics, user studies are one method of evaluation that allows researchers to validate the design choices and confirm or reject their hypotheses [27]. In this Thesis, a visual analytics approach was presented to support the exploration and analysis of complex environmental data. The goal of the evaluation was to assess the value of the approach and to understand how the system supported visual analysis and reasoning about the data. It ensured that the proposed approach addressed the previously identified challenges in the analysis of environmental data. Additionally, the field trial methodology used in this user study provided an understanding how domain experts use the system to explore within environmental data.

A number of hypotheses were prepared to predict user performance, validate the approach, and guide the evaluation. The user study evaluation focused on how environmental analysts use the system to explore and analyze the data. This user study provides an understanding of the usability and effectiveness of the system and its different features 
with respect to the analysis task analysts are performing. Qualitative methods based on field observations and interviews were used to assess how the system supports analysts in knowledge discovery and decision making.

The evaluation makes basic assumptions about the software, the data, and the participants. The first assumption is that the software and all the features are working correctly. The second assumption is about the data, the evaluations assumes that the data is correct without any mis-labelled data points or values. To evaluate how experts will use the system, it is assumed that the participants have at least general knowledge in the domain of the data.

\subsection{Hypotheses}

Several evaluation methods are widely accepted to evaluate information visualization systems [27]. In the absence of a baseline or similar systems, researchers have used multiple techniques to guide their evaluation process [83]. Field trial studies are evaluations that are conducted by studying how target users will use the system in a real work setting and with real users. By studying real users' activities, researchers are able to understand the system and improve their visualizations and approaches. In this Thesis, a field trial study with domain experts was conducted to evaluate the proposed approach. The user study evaluation was guided by three main hypotheses about how effective the approach is in addressing the main challenges of data analysis. To assess and measure these hypotheses, participant' feedback was assessed using multiple measures adopted from various information visualization evaluation methodologies [83, 92]. The extension of the Technology Acceptance Model (TAM2) [123] was also used to measure participant experiences. This evaluation process was guided with hypotheses focused on data exploration, knowledge discovery, and user experience. The details of each of these hypotheses are as follows: 


\section{Hypothesis 1 (H1):}

\section{The system supports data exploration of high dimensional heterogeneous geotemporal}

data. One of the fundamental goals of this research was to support the visual exploration of complex data. Exploring the data plays an important role in understanding and gaining insights about such complex datasets. This hypothesis mainly states that GCPC supports the exploration of high dimensional heterogeneous geotemporal data.

GCPC was designed to support users to explore different subsets and aspects of the data. Displaying the data on the parallel coordinates and geovisualization allows users to explore the different aspects simultaneously. The multiple filtering and navigation interactions in both views permit users to select and investigate different subsets of the data. As multiple filters may be applied at the same time, the system provides users with multiple tools to explore and select complex subsets of the data. The coordinated interactions between the multiple views support exploration of the different aspects of the data. Additionally, the investigative scatterplot facilitates exploring and inspecting the correlations between the dimensions.

\section{Hypothesis 2 (H2)}

The system supports knowledge discovery of high dimensional heterogeneous geotemporal data. Understanding high dimensional heterogeneous geotemporal data is a challenging problem. Allowing users to analyze the data and gain insights about the data plays an important role in knowledge discovery. GCPC was designed to support multiple analysis activities through the various features in the system. The multiple interactions available in parallel coordinates allow users to analyze the high dimensional heterogeneous data. The coordinated interactions between the parallel coordinates and the geovisualization helps users to identify patterns and relations within the complex data. The investigative scatterplot allows users to examine the correlations between the different factors. 
One of the main design goals of developing GCPC is to support the visual analysis of complex environmental data. The system analyzes the data to format and compute the initial statistical information. After this first analysis, GCPC displays the data in the parallel coordinates and the geovisualization. Users are able to filter, zoom, and interact with the system to analyze the data. Further analysis tools are provided to the user to support their analysis. To allow user to confirm or reject their findings, the system provides details when users hover or select a specific data point.

All these analysis tools provided in the system should permit users to confirm known information and discover new unexpected knowledge. With the design of GCPC focusing on analysis of complex environmental data, the expectation is that GCPC supports discovering knowledge in such data.

\section{Hypothesis 3 (H3):}

The system provides a good user experience while analyzing high dimensional heterogeneous geotemporal data. Users' abilities to use the system effectively and easily is the main requirement for adopting any new technology. In visual analytics systems, users' interactions with the system is one of the main components of the system. To visually analyze data using the system, GCPC must provide a good user experience. This hypothesis is divided into three sub hypotheses as follows:

\section{H3a: The system is useful to analyze high dimensional heterogeneous geotemporal}

data. The perceived usefulness is defined as the extent to which users' believe that using a system will help with their tasks [123]. This perceived usefulness impacts the experience users have with the system. The usefulness of GCPC is determined by how users believe that the system supports the analysis of the environmental data. As one of the main goals of this work is to support the analysis of complex environmental data, the perceived usefulness of GCPC is an important hypothesis to validate. 
GCPC employs a visual analytics approach to support users in their analysis tasks. Using the various interactions and analysis tools in GCPC, users are able to visually explore and analyze the complex data. The system provides multiple visualizations and interaction techniques to support the analysis of the various aspects of the high dimensional heterogeneous geotemporal data. The coordination between the parallel coordinates and the geovisualization allows for understanding the different relations within such complex data. In order to support the heterogeneous data types, the system visually encodes and handles the different data types in different ways. Additionally, the micro-visualization of statistical information helps users to understand the distribution of the data.

\section{H3b: The system is easy to use to analyze high dimensional heterogeneous geotem-}

poral data Users' abilities to use the system easily to achieve their goals is another aspect of user experience. The ease of use is defined by the degree to which a user believes that using the system is effortless [123]. The interaction and analysis in GCPC must be easy to use in order to allow users to focus on the analysis of the complex datasets.

GCPC was designed to be user friendly and provide intuitive interactions for the users. With the multiple visualizations developed to be effective, users should be able to quickly understand and correctly interpret the presented data. The system employs familiar interfaces to implement the interactions used in the system. For example, the system provides easy to use click and drag to filter the data in the parallel coordinates. To reorder the dimensions in the parallel coordinates, the system uses the familiar drag and drop to allow users to easily change the location of one coordinate in the parallel coordinates. To change the visual encoding of the data, users need to simply click on the controls below each coordinate. Similar to other interfaces, tooltips and additional details are displayed by hovering over the elements in the interface. When a user hovers over a point in GCPC, the point is 
highlighted and a tooltip is displayed providing additional details. This use of familiar interactions and interface elements allows users to effortlessly use the system in their analysis activities.

\section{H3c: The system provides a good output quality and dissemination of information}

Explaining the results and findings of the analysis to other collaborators and researchers is essential in any research. The system should provide good quality output, which can be easily understood by all the stakeholders. To design effective visualizations that are correctly perceived by users, information visualization concepts should be applied during the design process.

This research employed various information visualization and visual analytics concepts in the design and development of GCPC. The colour scales used in GCPC were selected with the awareness of the opponent process theory of colour [61] and how human perceive colour. While quantitative and ordinal data are encoded by a perceptually ordered colour scale, qualitative data are encoded using a perceptually distinct colour scale. The Gestalt similarity principle [77] was employed to ensure the correct interpretation of the patterns across the various views in the system. This effective use of visual encoding allows researchers to easily communicate the findings, which supports the dissemination of information.

\subsection{Evaluation Methodology}

Due to the complexity of visual analysis of the data, evaluating information visualization and visual analytics systems is a challenging problem. The evaluation of such systems not only assess the software, but also the users and their activities while using the software [27]. Choosing the best evaluation method to use depends on the type of analysis task, the type of system, and the domain of the analyzed data [27]. Evaluation methods may be 
categorized as either qualitative, quantitative, or a combination of both methods [27, 107]. Currently, various evaluation methods are accepted to assess and validate visual analytics systems. Researchers are encouraged to use multiple evaluation methods to provide a better understanding of their systems and strengthen their evidence [27, 63, 107].

As visual analytics systems are designed to support the analysis of data from specific domains, one of the challenges of choosing an evaluation method is the availability of domain experts to assess these systems. Another problem to consider when selecting an evaluation method is the availability or absence of baseline systems to compare against. In this work, qualitative evaluation methods were used to evaluate the system. A field trial methodology was used to conduct a user study in a realistic setting with a small group of domain experts. This methodology allows investigators to observe users' activities to provide an understanding of how they use the system. Additionally, open end analysis tasks can be easily used in field trials, allowing users to explore and analyze data in a more realistic setting.

In the field trials, two real environmental datasets were presented to the domain experts to study how analysts used GCPC to analyze and understand the data. To realistically validate hypothesis H1 (i.e., GCPC supports exploration of data) and H2 (i.e., GCPC supports discovering knowledge), the field trial used open end analysis tasks. Users were asked to freely use the system to explore and analyze the provided datasets in any manner they wanted. The field trials were conducted in Annapolis, MD, within an interdisciplinary research group using data collected by Pollnac et al. [99, 100]. As the field trials was organized at a research meeting, the study was conducted as a focus group. The focus group allowed the investigator to measure the use of the system in a collaborative setting with domain experts conducting group analysis of the data.

During the field trials, the investigator collected data in the form of: questionnaires, 
group discussion, video and audio recordings, and observations. The analysis of the collected data provided an understanding of the analysis activities of the researchers, the effectiveness of the system, and the usefulness and ease of use of the software. With the absence of baseline systems and the small number of participants in the study, quantitative analysis and evaluation of the system was not possible. Qualitative analysis of the use of GCPC is more relevant to the field trial methodology because of the participants' domain experiences and the limited number of individuals participating. Several qualitative analysis methods were used to interpret the collected data (details are in Section 4.3.4). Even with a small number of participants, the evaluation with real analysts provided trust-worthy evidence about the real value of the system.

\subsubsection{Coastal Resilience Data Set}

Environmental systems are characterized by multiple complex relations between various social, ecological, and economical factors. These multiple complex factors and relations drive researchers to develop environmental management solutions that can handle this complexity [31]. Coastal environments are an example of environmental systems that are influenced on different scales by multiple social, economical, ecological, and oceanographic factors [95]. The various natural and man made hazards that impact coastal environment, are a growing concern for reducing vulnerability and increasing the resilience of these environments [95].

Coastal resilience research focuses on improving responses and reducing vulnerability to natural and man hazards in coastal environments. It is a problem that requires an understanding of the multiple social-ecological factors that could directly or indirectly influence these environments. In this evaluation, environmental data from the field of coastal resilience were used in the field trial. 
The data were provided by environmental researchers, collected from 45 coastal communities adjacent to multiple Marine Protected Areas (MPA) in the Philippines [99, 100]. The data describe various factors, providing an overview of the economical, social, ecological, and other environmental aspects of these communities. The data provides details about the population, villagers' activities, households, fishing gear, various threats, MPA violations, etc.

Two different datasets were provided by the researchers, both both having details about the same coastal communities. The first dataset contained 220 dimensions measured for each of the 45 communities. The second dataset presented an additional 50 dimensions containing a different set of factors for the same communities. Even though the second dataset had a different set of dimensions, the data types in both datasets were the same. The data contained a mix of quantitative, qualitative, and ordinal data types. Geospatial data in the datasets consisted of the address of the village or community and the actual geographical location information. Although there were no specific temporal dimensions in these datasets, the data contained multiple dimensions that could be associated with a specific temporal periods (e.g., the population of the village at different time periods such as: population in 1985 , population in 1990 , and population in 1995).

\subsubsection{Study Procedures}

Before the beginning of the field trials, informed consent was obtained from all the participants. As part of the consent process, the investigator explained the goals and the procedures of the study.

The study was divided into multiple phases: pre-task questionnaire, presenting the system, using the system to analyze the data, post-study questionnaire, and open group discussion. While the study was conducted as a group activity, the study employed an individual data collection approach. To allow participants to assess the software individually, all the 
participants in the field trials had a chance to drive the software.

At the beginning of the study, the investigator asked all participants to answer a pre-task questionnaire to get demographic information about the participants. The questionnaire was designed to obtain information about the participants' experiences with coastal environment domains, data visualization, geographic information systems, and similar software systems.

During the demonstration of the system, the investigator introduced GCPC to the participants and gave a short demonstration of the key features of the system and how to use them to analyze environmental data. A sample dataset (i.e., same dataset used in Section 3.3) was used to explain how to use the system to explore and analyze the different aspects of the data. Participants were encouraged to ask questions about anything in the system or try to operate the system by themselves.

After demonstrating the system, the investigator provided the group with the two environmental datasets used in the study. The group started exploring and analyzing the data in an open ended analysis. As the study was conducted as a focus group, participants were given the opportunity to take turns using the software. While the participants were using the software, the investigator observed the participants' activities and assisted them using the software when they needed. This was followed with a similar group analysis of the second dataset with a larger number of dimensions. During the use of GCPC, the discussion and use of the system was video and audio recorded. This data was used by the investigator to analyze participants' performance and their interactions with the system.

After the group finished with the two analysis activities, each participant was asked to complete a questionnaire about the system and its key features. The questions focused on measuring their impression of the exploration and analysis ability, and the usefulness and ease of use of the main features of the software. The questions to measure users exploration and analysis were adopted from research on qualitative evaluations of information 
visualization systems [83, 92]. These questions were selected to measure the exploration and analysis activities of the users to validate the evaluation hypotheses ( $\mathrm{H} 1$ and $\mathrm{H} 2)$. The questions about the usefulness and ease of use were adopted from the extension of the Technology Acceptance Model (TAM2) [123], which is a model commonly used to verify hypotheses about the user's experience $(\mathrm{H} 3)$. In order to help the participants answering the questionnaire, the questions were divided into groups and a five point Likert scale was used to measure their responses.

In the final step of the study, the investigator initiated a group discussion about the software. The participants were given an opportunity to elaborate on their impressions of the positive and negative features of the system, how they think the system might support analysis of environmental data, and how they might be able to integrate the system in their work.

\subsubsection{Participants}

The study employed convenience sample from within a specific interdisciplinary environmental science research group. The participants were multidisciplinary research collaborators from Canada, USA, and Australia as part of a socio-environmental research group. The research group's activities are focused on coastal environment and methods to improve coastal resilience. The participants represented domain experts who were able to provide valuable insights into the usefulness of the system, ease of use, and how effective the system is in supporting exploration and reasoning for their analysis tasks on environmental data. The investigator invited members of the research group and the participants agreed to participate voluntarily in the study.

Five participants denoted by Participant 1, Participant 2, Participant 3, Participant 4, 
Participant 5 participated in the study. Table 4.1 shows participants' responses to the questions in the pre-task questionnaire. Participants had moderate to high experience with environmental data analysis, and a board range of experiences and prior knowledge with coastal environment domain and GIS tools. Additionally, three participants show moderate knowledge of visualization and visual analytics tools.

The pre-questionnaire provided adequate information for the investigator to understand the differences in analysts responses. As most participants were experts within the domain of environmental data analysis in general, their use of the system in the field trials provided an estimation of using the system in real world tasks. With the use of real datasets and

Table 4.1: Demographic of participants in the field trials

\begin{tabular}{|l|l|l|l|l|l|}
\hline $\begin{array}{l}\text { Experience with } \\
\text { environmental data } \\
\text { analysis }\end{array}$ & P1 & P2 & P3 & P4 & Pigh \\
\hline $\begin{array}{l}\text { Experience with the target } \\
\text { domain (i.e., Coastal } \\
\text { Environments/Resiliency) }\end{array}$ & High & High & High & Low & Low \\
\hline $\begin{array}{l}\text { Experience with } \\
\text { Geographic Information } \\
\text { Systems (GIS) }\end{array}$ & High & Low & Low & Medium & High \\
\hline $\begin{array}{l}\text { Experience with } \\
\text { visualization systems and } \\
\text { methods }\end{array}$ & High & Medium & Medium & High & Medium \\
\hline $\begin{array}{l}\text { Experience with analyzing } \\
\text { environmental data using } \\
\text { visualization systems }\end{array}$ & Medium & Low & None & Medium & Medium \\
\hline $\begin{array}{l}\text { Experience with parallel } \\
\text { coordinate plots? }\end{array}$ & Medium & Low & None & Medium & Low \\
\hline $\begin{array}{l}\text { Preferred tools to analyze } \\
\text { data }\end{array}$ & $\begin{array}{l}\text { GIS, } \\
\text { remote } \\
\text { sensing, } \\
\text { and R }\end{array}$ & $\begin{array}{l}\text { Range of } \\
\text { statistical } \\
\text { geospa- } \\
\text { tial } \\
\text { tools }\end{array}$ & $\begin{array}{l}\text { Statisti- } \\
\text { cal } \\
\text { software } \\
\text { and } \\
\text { Nvivo }\end{array}$ & $\begin{array}{l}\text { Develop } \\
\text { my own } \\
\text { tolysis }\end{array}$ & $\begin{array}{l}\text { Regres- } \\
\text { sion and } \\
\text { GIS tools }\end{array}$ \\
\hline
\end{tabular}


experts within the domain of the data, their activities showed real world analysis and their responses are considered reliable.

\subsubsection{Data Analysis Methods}

During the study, different types of data and feedback were collected from the participants. These included: post study questionnaires, focus group discussions, and investigator observations. Each data type required a different analysis and results presentation approach.

The post study questionnaire consisted of multiple measures to obtain users' feedback about using the system to analyze the data. The questionnaire collected this feedback for the system as whole and for each of the main features in the system. These features are: the parallel coordinates, the geovisualization (i.e., dot map and hex-binning), the microvisualization of statistical information, the interactive analysis features (i.e., the investigative scatterplot and dynamic encoding), and the outlier detection. The questionnaire was divided into multiple sets of questions for each measure (see the full questionnaire in Appendix $\mathrm{B}$. Table 4.2 shows the number of questions for each measure in the questionnaire. To asses user's perspective about the test hypotheses, each hypothesis was evaluated using one or more of these measures. To evaluate each feature and analysis tool in the system, the full set of questions and measures were repeated for each of these features, following the TAM2 framework [123].

As the study was conducted as field trials study with an open-ended use of the system, each participant analysis and use of the system was different. While controlled task each participant performs the same analysis task, in the open-ended use of the system every participant is free to perform their own analysis. Due to this uncontrolled nature of the field trials study, aggregating the participants' responses is not appropriate. Additionally, participants used the various features in the system in different ways, thus it is not suitable to aggregate the responses for the different features. Thus, the responses of each of these 
features are analyzed and presented separately. Participants' responses were collected by a number of questions for each measure using a 5-point Likert scale. The percentage of each response for each participant was computed for each measure. All the results for each feature are displayed in a divergent stacked bar chart [102] and presented in Section 4.4.1, Section 4.4.2, and Section 4.4.3.

During the group analysis of the data, the participants were engaged in an open group discussion. To allow the investigator to gain more understanding of the participants' activities, these discussions were audio and video recorded. The investigator analyzed the different opinions and responses by grouping the similar responses and coding them into categories [36]. The responses were coded based on the type of the comment as: negative responses, positive responses, and responses suggesting new features. A detailed analysis of these responses is discussed in Section 4.4.4.

In addition to participant responses, their activities and interactions with the system were also video recorded. The recording was used to observe and analyze how the participants used the system to analyze the environmental data. These activities provided further

Table 4.2: Detailed information about each measure used in the evaluation

\begin{tabular}{|l|l|l|l|}
\hline Measure & $\begin{array}{l}\text { Number of } \\
\text { questions }\end{array}$ & Hypothesis & Reference \\
\hline $\begin{array}{l}\text { Perceived usefulness of the } \\
\text { system in data exploration }\end{array}$ & 5 & H1 & $\begin{array}{l}\text { Adopted from information } \\
\text { visualization evaluation } \\
\text { literature [83, 92] }\end{array}$ \\
\hline $\begin{array}{l}\text { Perceived usefulness of } \\
\text { the system in the detailed } \\
\text { data analysis and } \\
\text { knowledge discovery }\end{array}$ & 9 & $\mathrm{H} 2$ & $\begin{array}{l}\text { Adopted from information } \\
\text { visualization evaluation } \\
\text { literature [83, 92] }\end{array}$ \\
\hline $\begin{array}{l}\text { Perceived usefulness (i.e., } \\
\text { general usefulness) }\end{array}$ & 6 & H3a & Adopted from TAM2 [123] \\
\hline Job relevance & 2 & H3a & Adopted from TAM2 [123] \\
\hline Perceived ease of use & 6 & H3b & Adopted from TAM2 [123] \\
\hline Output quality & 6 & H3c & Adopted from TAM2 [123] \\
\hline
\end{tabular}


understanding on how the different features in the system were used to explore the data. Section 4.4.5 explains the investigator's observations about the participants' activities

\subsection{Results}

Using the post study questionnaire, each of the evaluated hypotheses was validated using one or more measures. The questionnaire consisted of 6 sets of questions for each of the following measures: perceived usefulness of the system in a general exploration of the data, perceived usefulness of the system in detailed analysis and knowledge discovery of the data, job relevance, perceived usefulness of the system as a whole, perceived ease of use, and output quality.

The next three sections explain how these measures were used to assess each hypothesis and discuss the results. Each hypothesis is presented in a separate section, which explains the results of GCPC as a whole and after that presents the results of each feature.

\subsubsection{H1: Data Exploration}

The first hypothesis is based on the expectation that data exploration is an essential element of visual analytics systems. To support this hypothesis, the system should allow users to explore the data in a variety of ways. GCPC is designed to allow users to explore the data through visual analysis processes by interacting with the parallel coordinates, the geovisualization, and the investigative scatterplot. Users can filter, zoom, and dynamically change the visual encoding of the data.

The responses the participants gave regarding the exploration of the data in the system are presented in Figure 4.1. Most participants' responses agree or strongly agreeing with statements related to the support of data exploration in GCPC. These generally positive responses show that the participants see the value of using GCPC to explore the data. 
A more detailed analysis of how the different features of GCPC supports exploration is illustrated by examining participants responses for the different features. Figure 4.2 presents the results of each of the main features in the system.

Similar to the overall system results, the participants' responses for parallel coordinates are positive. Figure $4.2 \mathrm{a}$ shows that responses are divided between agreeing and strongly agreeing with the statements about the usefulness of parallel coordinates in exploring the data.

Similarly, Figure $4.2 \mathrm{~b}$ shows that the majority of the participants agree or strongly agreeing with statements confirming the usefulness of exploration using the geovisualization. Participants were able to view, filter, and explore the geospatial data displayed in the geovisualization.

The similarity of the participants' responses for the GCPC, the parallel coordinates, and the geovisualization indicates that the usefulness of these two views impacted the results of the whole system. As the parallel coordinates and the geovisualization are the two main components of the system, this is understandable. While exploring the data in the field trials, participants focused on using the parallel coordinates to examine different subsets of the data and used the geovisualization to investigate the geospatial relation of the high dimensional data.

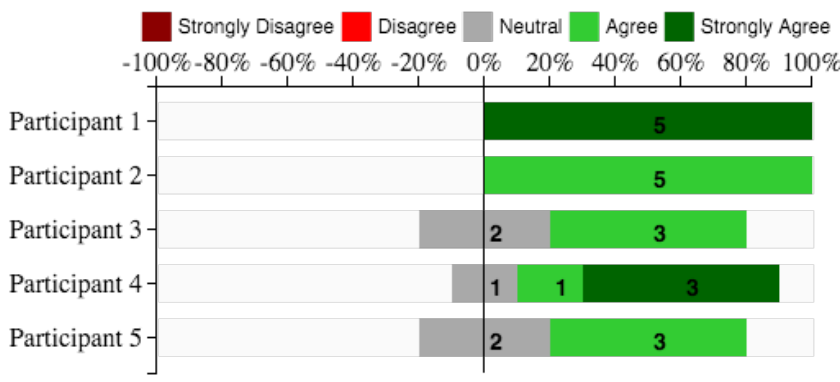

Figure 4.1: Perceived usefulness of GPCP in data exploration 


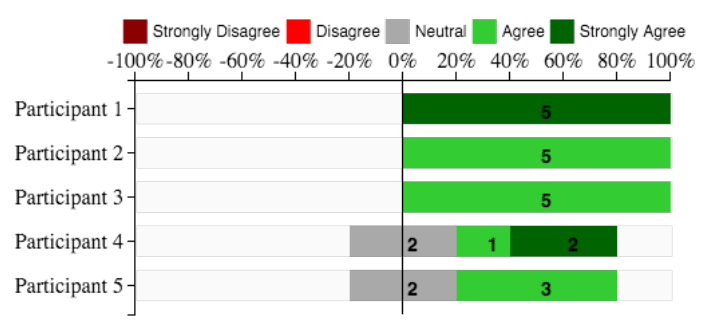

(a) Parallel coordinates

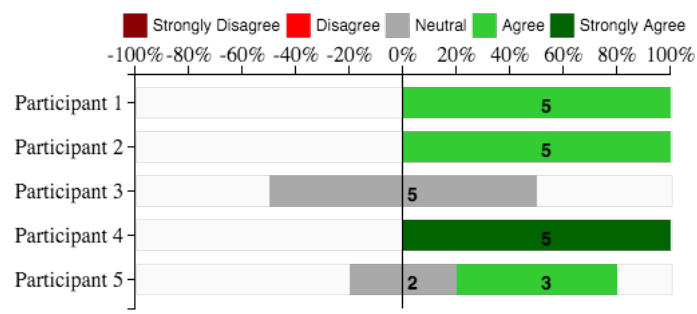

(c) Micro-visualization of statistical information

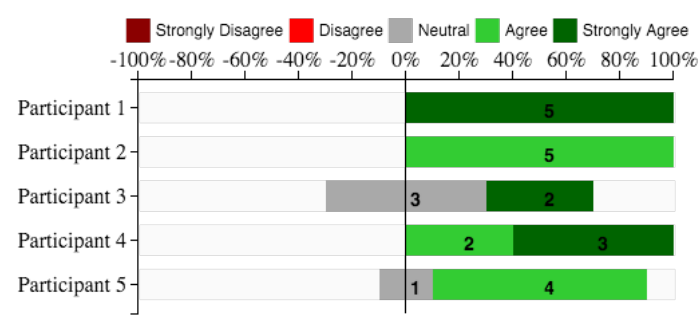

(b) Geovisualization

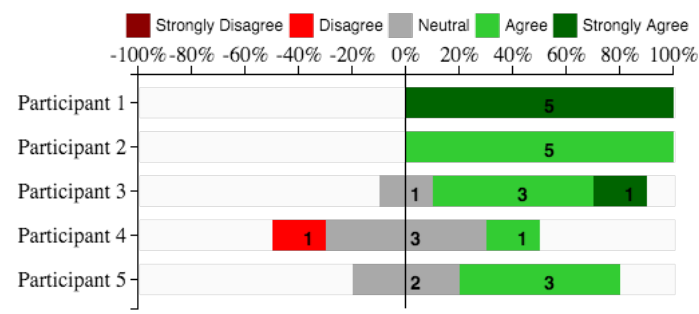

(d) Investigative scatterplot and dynamic encoding

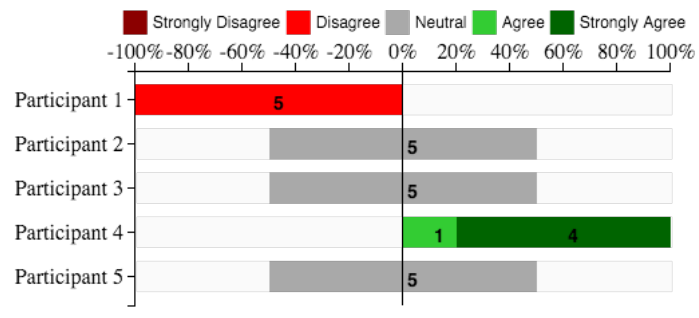

(e) Outlier detection

Figure 4.2: Perceived usefulness of the different features in data exploration

Figure 4.2c shows that only one participant (Participant 3) gave neutral responses about the exploration usefulness of micro-visualization of statistical information, while all other participants tended to agree or strongly agree with exploration of this feature. The neutral responses of Participant 3 is a result of this individual not using this specific feature, as the participant was more concerned with using the parallel coordinates and the geovisualization. Additionally, Participant 3 was very experienced in using professional statistical analysis tools, which could indicate some disappointment at not seeing advanced statistical tools in this prototype. 
Figure $4.2 \mathrm{~d}$ shows that Participant 4 had mixed responses with the statements for the analysis features. Participant 4 was an expert user of visualization software so this individual perhaps had a higher expectation of this GCPC prototype. Another reason for mixed responses could be because the investigative scatterplot and dynamic encoding are mainly features that support the analysis rather than an exploration of the data.

As shown in Figure 4.2e, the participants had mixed responses on the outlier detection feature in supporting exploration of the data. The result is explainable as distrust of the detection algorithm was openly expressed by Participant 1 during the field trials. This resulted in other participants distrusting the automatic detection algorithm as well.

These responses show that the participants saw the value of using GCPC in general to explore environmental data, supporting the acceptance of Hypothesis 1. The results also show that some features support the hypothesis and exploration of the data more than others.

\subsubsection{H2: Knowledge Discovery}

Hypothesis 2 is based on the principle that discovering knowledge is a challenging process that involves locating new information, identifying patterns and trends, and understanding the different correlations. With the integration of multiple coordinated views and various analytical tools, GCPC was developed to support a knowledge discovery process through a visual exploration of the data.

The participant responses to questions regarding the analytical ability of GCPC approach are reported in Figure 4.3. The figure shows a diversity in participants responses about this measure. The majority of the participants agree that GCPC is useful in data analysis and supports the knowledge discovery process. Participant 1 was in strong agreement regarding the benefits of the system in the analysis of data. Other participants saw 


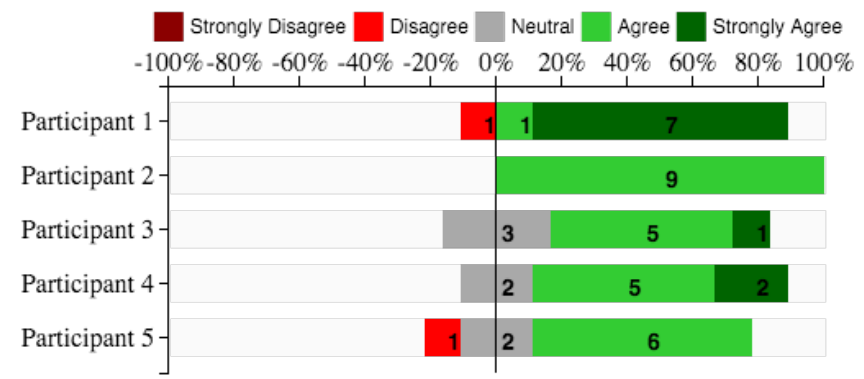

Figure 4.3: Perceived usefulness of GPCP in detailed data analysis and knowledge discovery

the value of using GCPC in exploring and investigating relations between high dimensional data. However, they indicated that GCPC is more valuable as an exploration tool or primary analysis tool. The participants explained that other professional statistical and analysis tools are better suited for detailed analysis of the data.

Comparing the different features of knowledge discovery, Figure 4.4 shows mixed responses from the participants. Figure 4.4a shows the results for the parallel coordinates. The weakest affirmation of this feature came from Participant 5, likely because of this individual's lack prior knowledge of the data.

Most participants agree or strongly agree with the usefulness of geovisualization in analyzing the data (Figure 4.4b). Notably, since Particpant 4 had prior experience in using advanced visualization tools, the participant had high expectation of the geovisualization analysis tools, and their responses ranged from disagreement to neutral.

The results of the micro-visualization of statistical information also show mixed responses. Figure $4.4 \mathrm{c}$ shows a mixture of moderate agreement with the statements, disagreement of some statements, and neutral responses. Notably, Participant 3 did not test this feature so provided a neutral response, consistent with the participant response in the previous hypothesis. However, all other participants tended to agree with the value of statistical information in analyzing the data. 


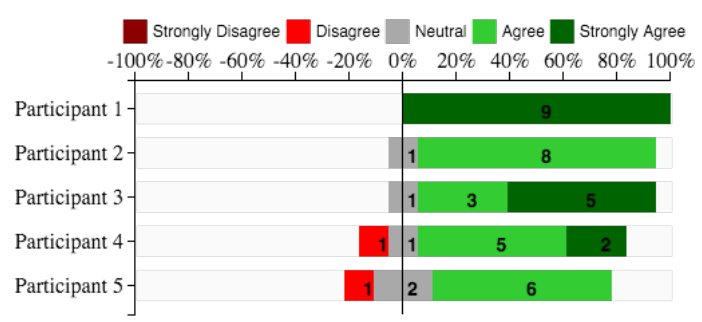

(a) Parallel coordinates

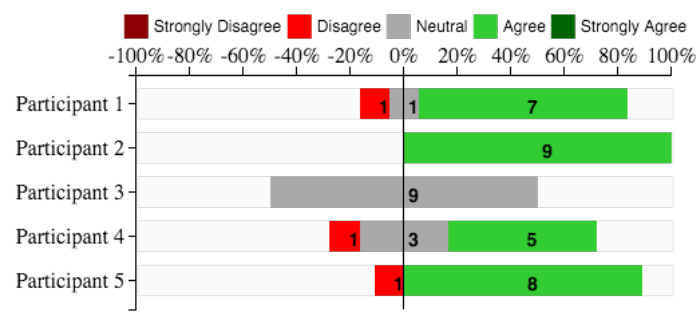

(c) Micro-visualization of statistical information

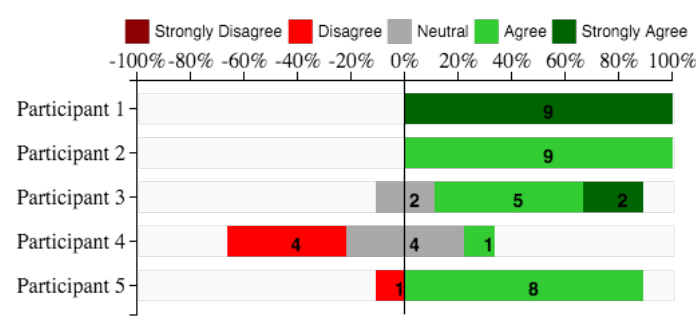

(b) Geovisualization

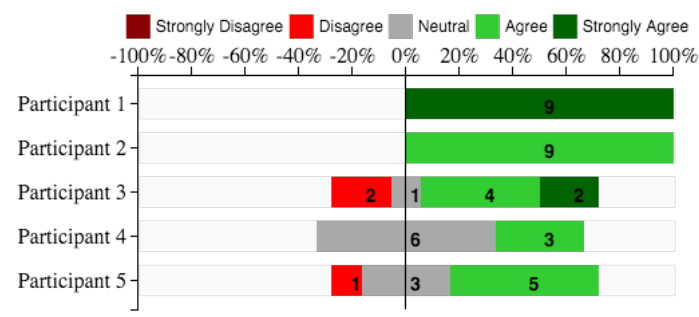

(d) Investigative scatterplot and dynamic encoding

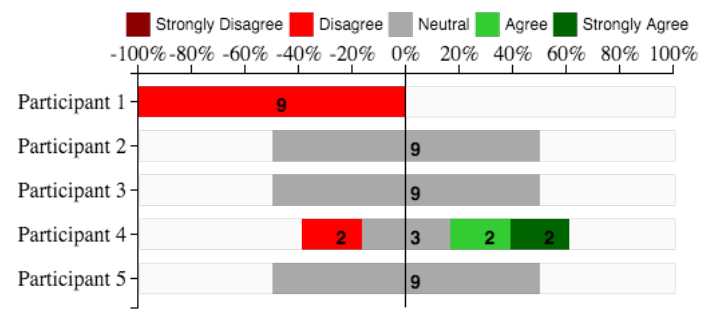

(e) Outlier detection

Figure 4.4: Perceived usefulness of the different features in detailed data analysis and knowledge discovery

Participants had mixed responses on the analytical abilities of the investigative scatterplot and dynamic encoding. Figure 4.4d details the participants' responses. The mixed responses for Participant 3 were likely due to this participant's experiences with professional statistical analysis tools. The participant commented that seeing a correlation in the system is different than understanding it, which requires external statistical analysis tools.

Participant responses to the analysis capability in the outlier detection show neutral and more disagreement than to other features in the system. Figure 4.4e shows that Participant 1 disagreed with the benefit of the outlier detection in analyzing the data and this influenced 
the responses from the other participants.

These responses show that the participants appreciated the benefit of using GCPC in an analysis of the data, but only as initial exploration tool. The results show that Hypotheses 2 is supported by the system in general, but some the separate features do not provide the same support for analyzing the data. This is understandable as the power of the visual analytics approach is the merging of multiple different visualizations and techniques to support users' analysis. The results also show that the outlier detection feature did not support the analysis of the data for these participant, and thus the hypothesis is rejected for this feature.

\subsubsection{H3: User Experience}

The user experience hypothesis was divided into three sub hypotheses that were measured separately using four measures. These measures were perceived usefulness, job relevance, ease of use, and output quality of the software.

\section{Perceived Usefulness}

Perceived usefulness was defined as the extent that users' believe the system is useful for their tasks [123]. The different features in the system were designed to facilitate user interaction with the system and aid their analysis of the data. Hypothesis 3a states that analysts will find the system useful. The extension of the Technology Acceptance Model (TAM2) [123] proposes that perceived usefulness is also affected by how relevant the system is for users' tasks. To assess H3a we asked participants about each of these measures.

Figure 4.5 and Figure 4.6 show the results of the job relevance and perceived usefulness of the GCPC system, respectively. The answers to the questionnaire show that all participants believed that GCPC is relevant to their analysis. The responses on the usefulness of the system were divided between agreeing and strongly agreeing to the usefulness of 


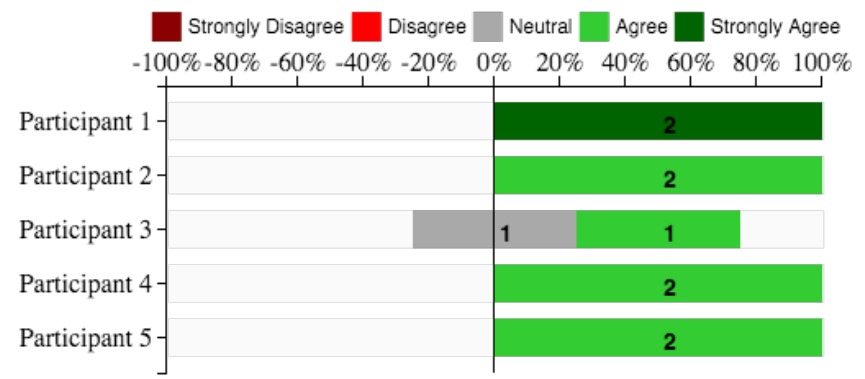

Figure 4.5: Job relevance of GCPC

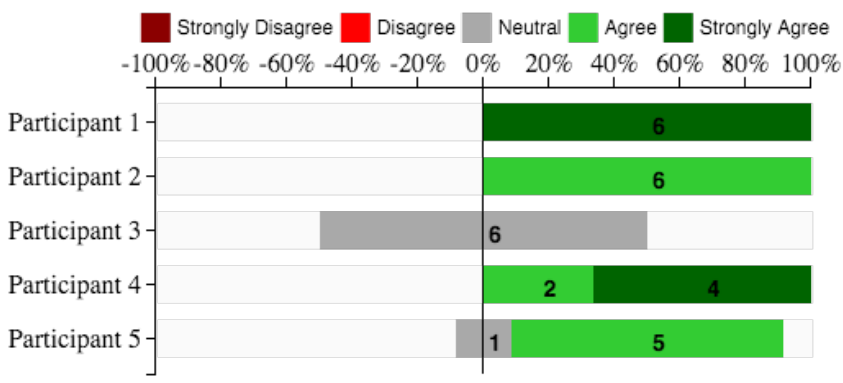

Figure 4.6: Perceived usefulness of GCPC

the system. The participants found it useful to use the system to explore and analyze the data. The interactive features in the parallel coordinates and the coordinations between the multiple views allowed participants to explore multiple relations in the data. Notably, Participant 3 provided more neutral responses, likely because of their familiarity with professional statistical analysis tools.

The responses to job relevance and usefulness of the different features are presented in Figure 4.7 and Figure 4.8 respectively. For the parallel coordinates, participants found it relevant to their analysis (see Figure 4.7a). Figure 4.8a shows that participants are tended to agree with the usefulness and effectiveness of the parallel coordinates in the data analysis. As previously explained Participant 3 viewed the using GCPC as primarily an exploration tool; the participant responses are more neutral in all the features. 


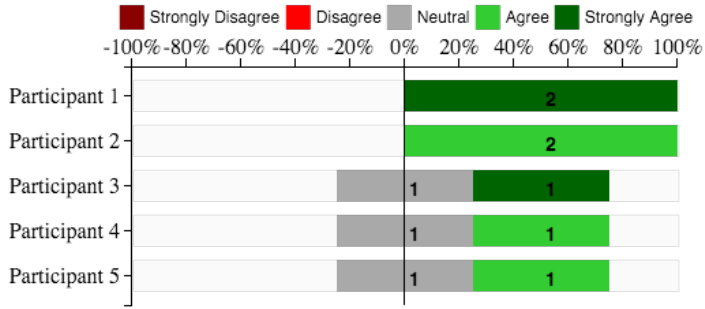

(a) Parallel coordinates

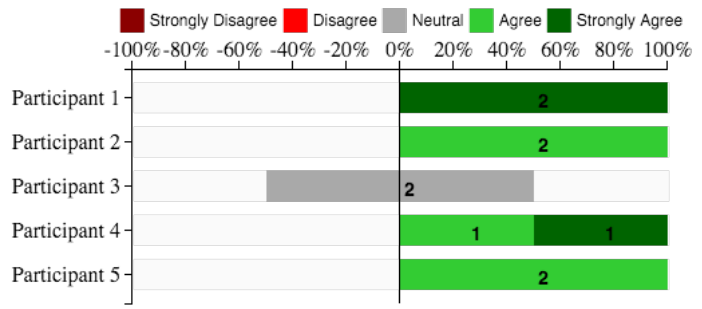

(c) Micro-visualization of statistical information

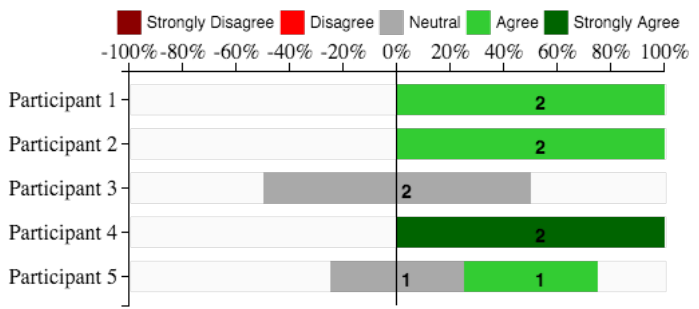

(b) Geovisualization

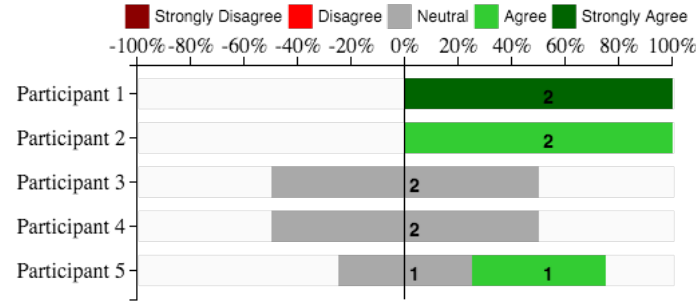

(d) Investigative scatterplot and dynamic encoding

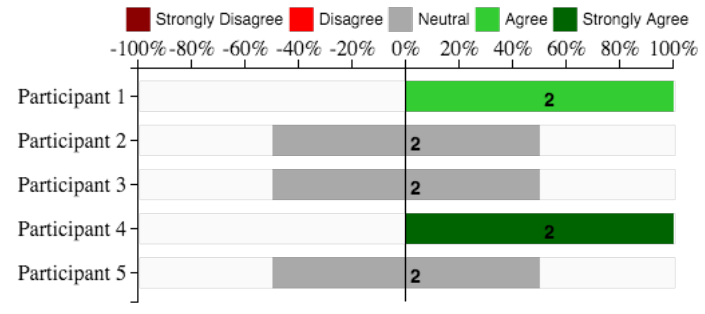

(e) Outlier detection

Figure 4.7: Job relevance of the different features

Similar to the results of usefulness of parallel coordinates, most participants agreed with the usefulness of the geovisualization feature (see Figure $4.7 \mathrm{~b}$ and $4.8 \mathrm{~b}$ ). Consistent results were also obtained for the micro-visualization of statistical information and the analysis tools (i.e., investigative scatterplot and dynamic encoding). With the exception of Participant 3, all participants found the micro-visualization relevant to the analysis (see Figure $4.7 \mathrm{c})$. From the data in Figures $4.8 \mathrm{c}$ and $4.8 \mathrm{~d}$, it is also noted that participants found the usefulness of the micro-visualization and analysis features to be generally positive. The results confirm the observations that the users were able to apply the various analysis 


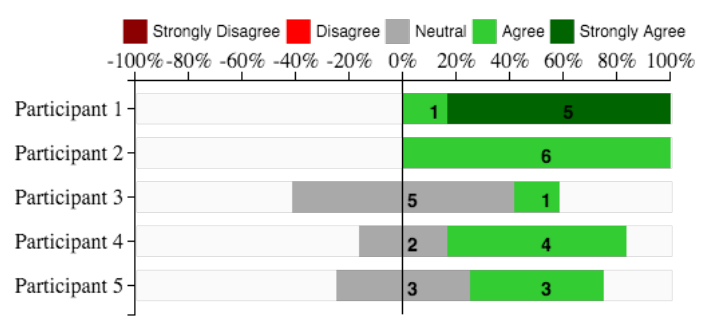

(a) Parallel coordinates

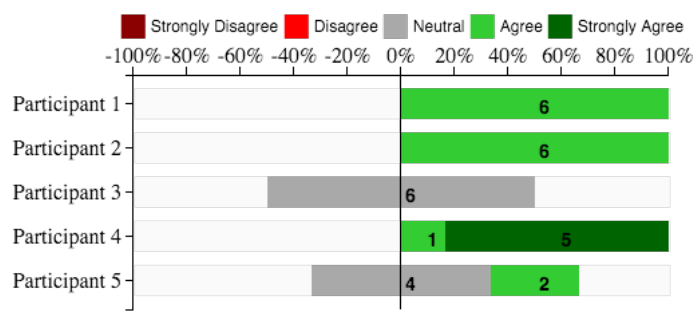

(c) Micro-visualization of statistical information

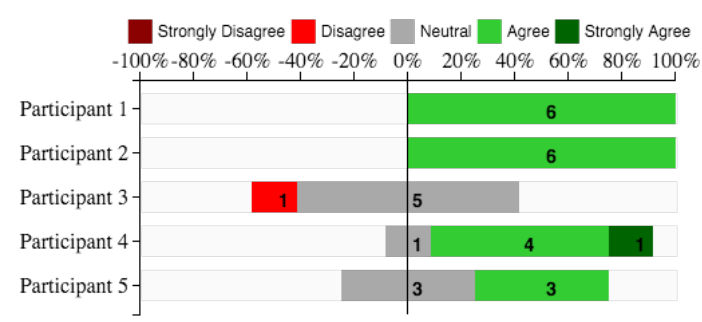

(b) Geovisualization

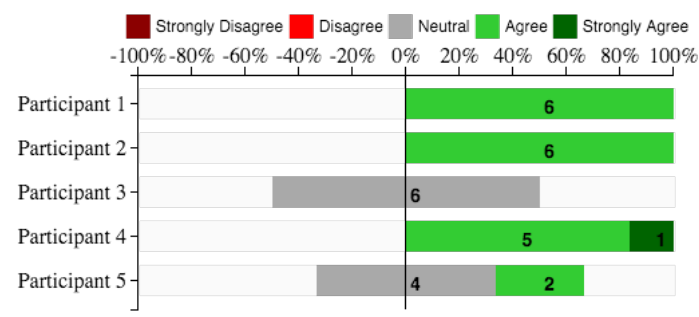

(d) Investigative scatterplot and dynamic encoding

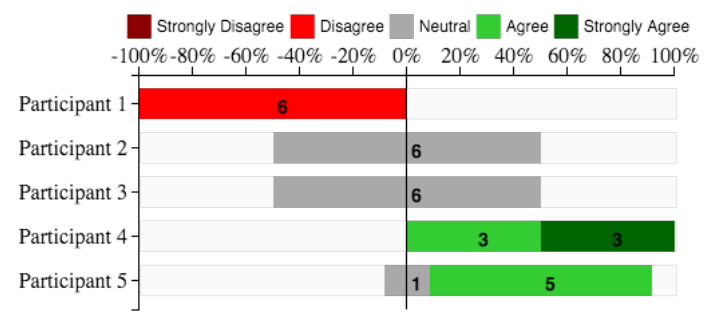

(e) Outlier detection

Figure 4.8: Perceived usefulness of the different features

features to understand and analyze the data.

The results in Figure 4.7e show that most participants have neutral responses about the relevance of outlier detection to their work. Figure $4.8 \mathrm{e}$ shows participants' responses regarding the usefulness of the outlier detection. Due to the expressed doubts about the effectiveness of the automatic algorithm and insufficient use of the outlier detection during the field trials, as described above, the mixed responses for the perceived usefulness of this feature are understandable.

These responses show that the majority of the participants believed that GCPC and 
the main features are useful. They found the system relevant to their analysis tasks and useful for the analysis of complex environmental data. Based on the responses from the participants, H3a is accepted. Additionally, the results also show that all the features, except the outlier detection, support the hypothesis.

\section{Ease of Use}

While the usefulness of the software is concerned with its helpfulness and effectiveness, the ease of use is more about how users believe that using the software is effortless. GCPC supports users in the analysis of complex environmental data using multiple visual interfaces and interactions. The system was designed to focus on user interactions with the system. Straightforward interactions are used in a web-based interface to facilitate the analysis of the data. Users can change encoding, reorder dimensions, filter and navigate to analyze the different aspects and subsets of the data. It was anticipated that participants would find the software easy to use during their analysis activities.

Observing the results in Figure 4.9, three participants agreed that using GCPC to analyze environmental data was effortless. The other two participates were neutral about the ease of using the software. Participant 5 was not familiar with the operating environment and this presented some difficulty in using the software. Overall, these findings support the hypothesis that GCPC is easy to use.

An analysis of the participants' views on the individual features provide more clear agreement on the ease of use than of the whole system. Figure 4.10 shows the participants' responses regarding the ease of use for each feature.

During the field trials, participants' activities focused on using the parallel coordinates and the geovisualization to explore the data. As the participants were able to interact with the parallel coordinates using familiar interactions, the perceived ease of use of the feature was mostly positive. Responses presented in Figure 4.10a show that most participants 


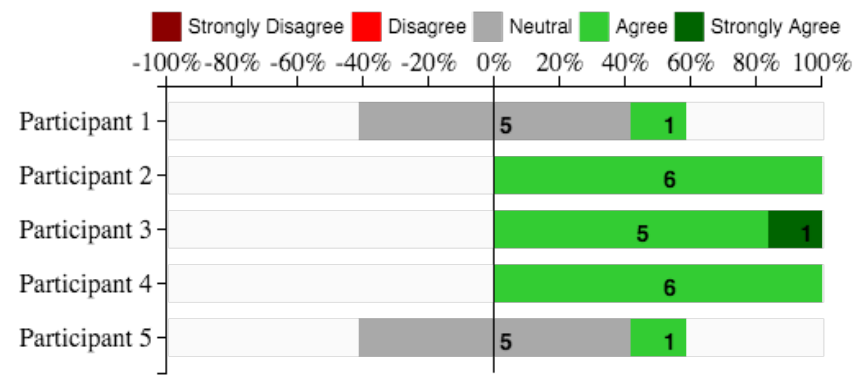

Figure 4.9: Perceived ease of use for GCPC

agreed with the ease of use of the parallel coordinates.

The result of ease of use of the geovisualization feature is similar to the parallel coordinates. Figure $4.10 \mathrm{~b}$ shows that all participants, except Participant 5, either agreed or strongly agreed with the ease of use of the geovisualization. Participant 5's responses indicate that the participant did experienced difficulty in operating the software, as it was not easy to use in an unfamiliar operating system.

The micro-visualization of statistical information feature was also perceived as easy to use by most participants. The results in Figure $4.10 \mathrm{c}$ show that participants were divided between agreeing and strongly agreeing with the ease of use of this feature. Participant 3 remained consistently neutral about this feature due to the participant's limited interaction with it.

Figure 4.10d illustrates the responses of the participants on the ease of use of the investigative scatterplot and dynamic encoding feature. The majority of the participants agreed that these analysis features were easy to use. Participants were able to easily use the scatterplot to explore relations between different dimensions.

Similar to other measures, the outlier detection features seemed to get mixed reviews, eliciting neutral and disagree feedback (see Figure 4.10e). As participants were not confident about the efficacy of the automatic algorithm and since the feature was not adequately 


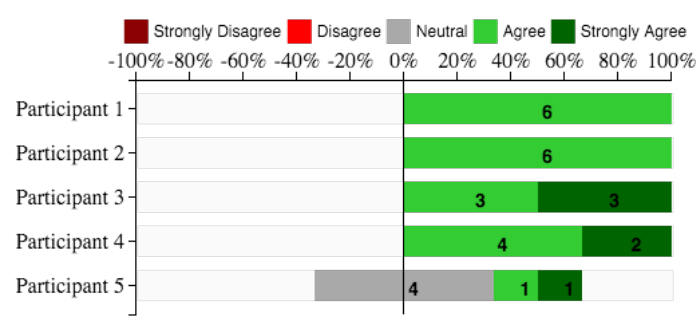

(a) Parallel coordinates

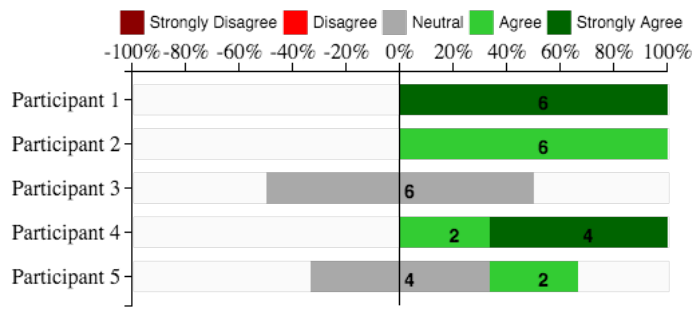

(c) Micro-visualization of statistical information

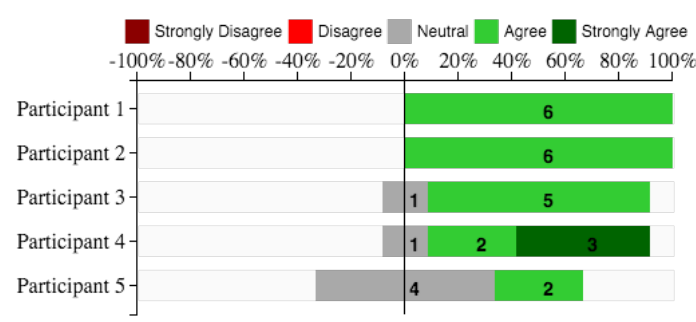

(b) Geovisualization

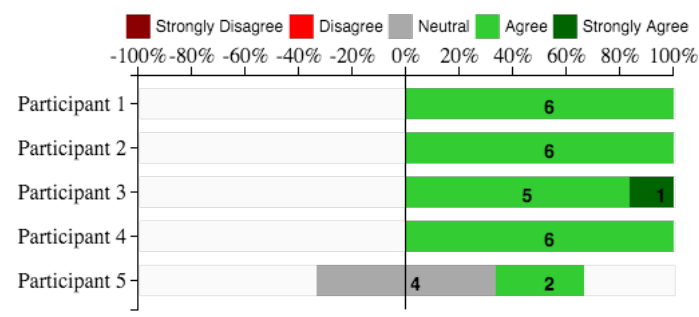

(d) Investigative scatterplot and dynamic encoding

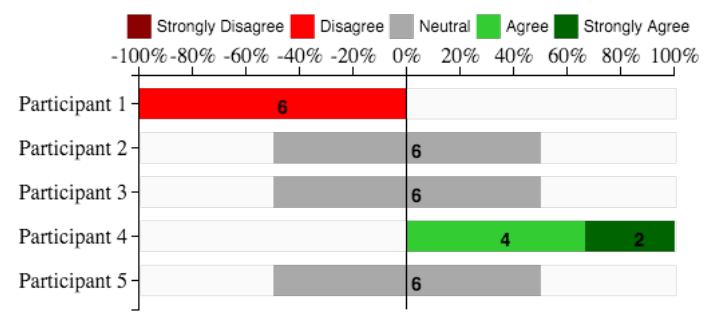

(e) Outlier detection

Figure 4.10: Perceived ease of use of the different features

tested, these results are understandable.

Overall, the results show that the participant found GCPC easy to use. All the features, except the outlier detection, evoked positive feedback on the ease of use of the features. These results suggest that the system is easy to use for analysis of environmental data. Thus, the results accept Hypothesis $3 b$. 


\section{Output Quality}

Hypothesis $3 \mathrm{c}$ states that the system provides a good output quality and dissemination of information. Users' perception of the output from the system plays a vital role in understanding the data they are analyzing. The quality of the output of the system is based on users' belief that the output of the software is useful for their analysis tasks and easy to explain to other parties. GCPC design choices were made with awareness of information visualization theories and concepts. The opponent process theory of colour [61] was used to guide the choice of colour scales used in the system. Additionally, the Gestalt Principles [77] were considered to ensure users' understanding of the patterns in the visualizations.

Responses to the output quality measures for GCPC are reported in Figure 4.11. The results show that the majority of the participants had positive feedback and agreed that the system has high output quality. Further analysis shows that Participants 4 and 5 had positive opinions on the quality of output of the system, but neutral responses to how easy it was to communicate this output. In contrast, Participant 3 expressed strong agreement to the use of the GCPC to explain and communicate the output.

Mixed responses are noticed in the answers to the output quality of parallel coordinates. The overall responses were positive, but Participant 2 and Participant 5 had a mix of

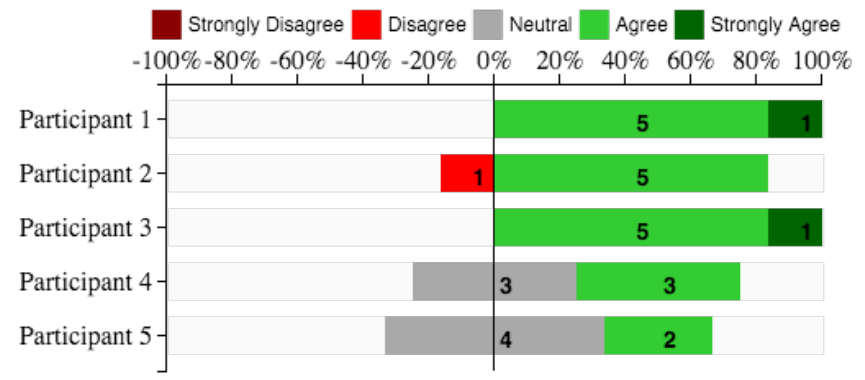

Figure 4.11: Output quality for GCPC 


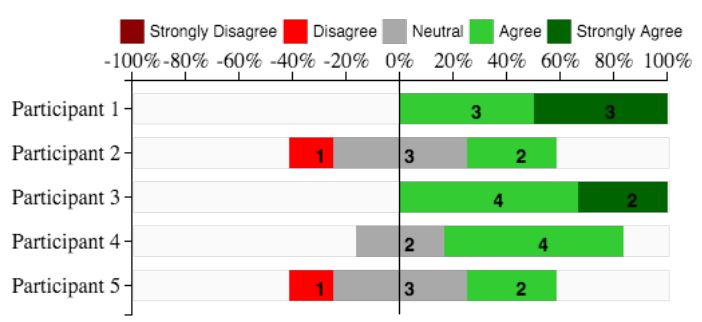

(a) Parallel coordinates

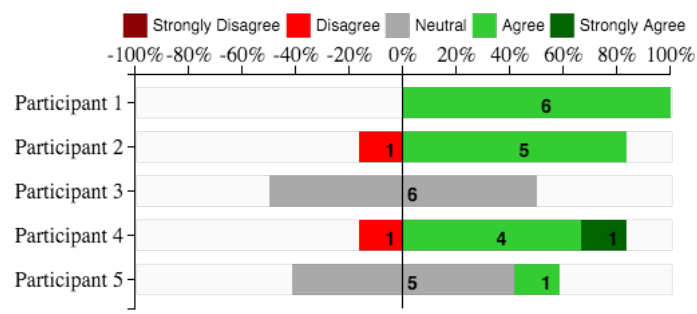

(c) Micro-visualization of statistical information

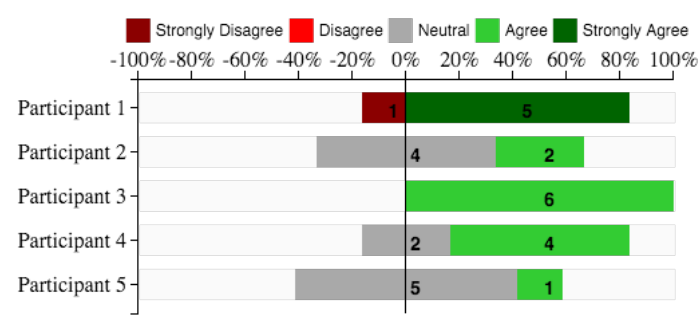

(b) Geovisualization

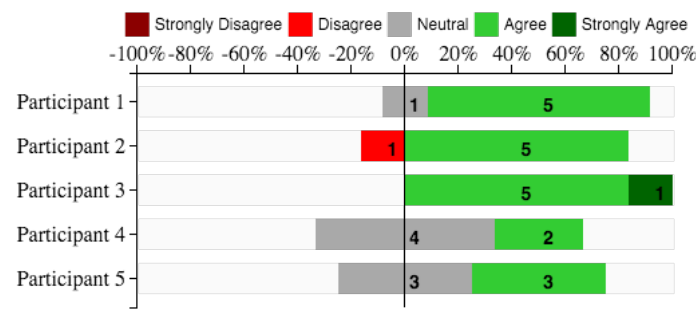

(d) Investigative scatterplot and dynamic encoding

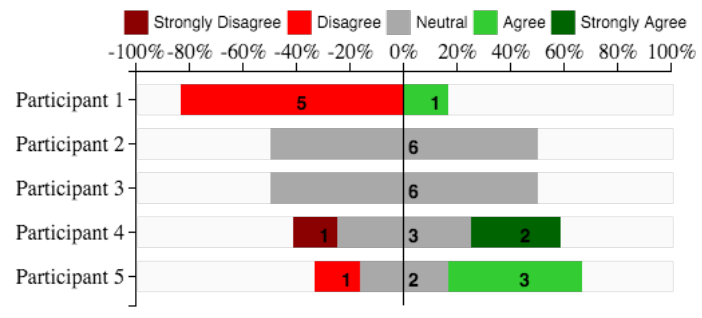

(e) Outlier detection

Figure 4.12: Output quality of the different features

disagreeing and neutral responses. These results are shown in Figure 4.12a

The results for the geovisualization are slightly better as the participants' responses tend toward agreeing with the high quality of the system output. Figure $4.12 \mathrm{~b}$ shows that Participant 1, Participant 3, and Participant 4 agree or strongly agree with the output quality of the geovisualization. These results are better than the parallel coordinates, which could be due to the experience of these participants in GIS systems and geographical interfaces. Figure $4.12 \mathrm{~b}$ also shows that Participant 2 and Participant 5 were cautious about the output quality and gave neutral responses. 
Participants' responses regarding the output quality of the micro-visualization of the statistical information feature were generally in agreement, but there were a few disagreements. Figure $4.12 \mathrm{c}$ shows the participants' responses for the output quality of this feature. While the majority of the participants agreed with the quality of the output, Participant 2 and 4 disagreed on the ability to explain the output of this feature.

The results presented in Figure 4.12d shows that the majority of the participants agreed on the output quality of the investigative scatterplot. Participants found the result of the investigative scatterplot easy to understand and communicate to other participant. The dynamic encoding of the data allowed participants to understand the distribution of the data points. These analysis features provided participants with high quality output that could be easily communicated to other parties.

On the other hand, the output quality of the outlier detection garnered negative responses, arising from doubts about the output of the automatic outlier detection algorithm (Figure 4.12e). With the exception of Participant 1, who disliked the quality of the output of the outlier detection, the other participants did not have strong opinions about this feature.

In summary, the results show that most participants believed that the GCPC produced high-quality output, in support of Hypothesis 3c. Since mixed responses were recorded for the individual features within the Output category, the hypothesis was neither rejected nor accepted for the different features.

\subsubsection{Focus Group Discussion}

Since this study was conducted as a focus group, the participants were engaged in unstructured discussion during their use of the system. After the group finished the analysis, the investigator initiated another semi-structured discussion regarding their opinions about 
the use of the system (see Appendix B for the discussion questions). Three types of comments were noted while analyzing these discussions. Participants had positive responses to the system and how they could use the system. The second type of response was negative responses about the difficulty of using the system. The final type of responses was suggestions for new features and improvements to the system.

\section{Positive Responses}

The analysis of the conversation during the group discussion provided qualitative feedback about participants' opinions of the system. The participants agreed with multiple comments about the system. All the participants were positively engaged with the system,

mentioning the benefit of the system data. Participant 2 commented on the value of the GCPC by saying it was " extremely valuable".

The participants agreed on the benefit of the system as a preliminary analysis tool. The participants saw the value of the system in exploration of the data more than an analysis tool. As Participant 2 put it "I see this as a discovery and exploration tool, then moving the data into spatial statistical package like GeoDa to look at [the] statistical relationships between variables." Participant 1 commented that "I think the way to really do this is if we can do an export to a geo-referenceable database, then [perform] analysis" and Participant 3 added "I would see it bing more [valuable on] the communication side rather than even analysis." Another participant agreed with this statement saying "that is what I see too". These responses from the participants provided further evidence for Hypothesis 1 , but less support for Hypothesis 2.

Participant 2 responding to the question of how they will use GCPC in their work mentioned that it could be used "to explore complex relationship". Participant 5 elaborated on the analysis ability to detect relations in parallel coordinates, saying "because there is so much flexibility, [moving] this [coordinate] to this [location], [the view] can change every 
single time. You can start looking for a much more complex relationship much more easily than you could [using other systems]".

As the analysis progressed the participants were asking about using the system in their work. Participant 3 stated that "to me this will be an awesome tool to be able to take to the Mekong River Commission and let them play with it." Participant 5 commented as "You can imagine this as driving some type of statistical approach, where you are using it to identify key [dimensions]".

\section{Negative Responses}

During the field trials, the participants faced some difficulties using the system. As the dataset consisted of more than 200 dimensions, selecting a subset of the dimensions to analyze was an exhausting task. Participants took a long time to search and find interesting dimensions to investigate. Participant 3 commented on the dimension labels "it is difficult to know what they are". Participant 4 suggested an improvement to list the dimensions alphabetically. However, Participant 5 replied that this is not the best representation nothing that" the problem with alphabetical ordering is that once they lose their friends above or below, it is actually a little bit harder to figure what some of them are". Another suggested adding a search feature. While this presented difficulty for the participants during the analysis, it was more an issue with the data itself and some missing meta information about the data.

Another difficulty was missing values in the dataset, which were interpreted in the system either as zero values or empty values (i.e., an empty string or '?') for qualitative data. The representation of missing values is a well known limitation of parallel coordinates because each data point is represented with a polyline intersecting the coordinates. In GCPC, missing information is replaced with Zero or an empty string according to the type of the data which allow them to be visualized in the parallel coordinates. However, this presented 
a problem as it misled the participants to investigate some points that they believed were interesting but were just results of missing information in the data. One participant noticed a pattern in the data asked "Why is there a large jump in the data" but after examining the details of the datapoints participants found it was missing information that created this pattern. After a similar investigation one participant commented "(missing data) is going to be a problem" another participant agreed, saying "we have to do something about missing data."

\section{New Features or Improvements}

While the participants used the system to analyze the data, they made comments on improvements and suggested features they would like to add to the system. These improvements are summarized as bellow:

Modifications were suggested included adding some additional improvements to the parallel coordinates to allow participants to fix issues in the dataset dynamically. For example, the data contained some dimensions that were wrongly identified as numerical even though they represented categorical data. Similarly, binary data that did not have metadata associated with it was identified as numerical data. A participant suggested a new function to change the data values interactively. Participant 5 said "you can just take the data and change the axes yourself, to just get rid of all the non integer values".

Another suggestion came from the geographical researchers to add more spatial context to the geovisualization. Participant 5 commented while referring to the MPA (Marine Protected Areas) near the villages in the datasets "I think we will need a separate map that just shows the MPA in the area." Participant 2 asked if there was a shapefile (i.e., a common file

type for geographical data) that could be used in the system. Participant 1 added a similar comment mentioning that there is no topology saying "I think we can overlay layers on here, but there is no encoding of topology, which we need [in order] to do spatial analysis." 
Another recommended improvements focused on the size of the selected or filtered subsets of the data. While the participants were filtering and highlighting different subsets of the data, the number of selected datapoints were not displayed. The participant thought this information could be useful and wanted to know the number of datapoints in their current selection.

\subsubsection{Observations}

While the group discussion provided important feedback from participants, observation of their activities while using the system provided an understanding of their choice of tasks. Due to the nature of the focus groups, participants used the system in a group analysis. However, each participant had the chance to drive the software and conduct the analysis activities . While the participants pursued multiple analysis tasks, the investigator observed the patterns in which they used the system.

Each analysis task started by the participant selecting a subset of dimensions from the full set of dimensions. Due to the large number of dimensions in the dataset, this step took a long time for each new analysis. After they selected the dimensions, the participants examined the parallel coordinates and tried to find obvious patterns in the data. After that, the participants tried to rearrange the axes of the parallel coordinates to gain more understanding of the correlations between their chosen dimensions. At this point an interesting pattern in the data began to emerge and the participant then began filtering down the data from one of the parallel coordinate dimensions.

With the filter applied to the parallel coordinates, participants usually wanted to get more details on the exact points. They focused their attention on the map and tried to get the details of each displayed point. The small number of data points helped the participant in this step and allowed them to check the details of each point easily.

While most of analyses used these steps, Participants 1 and 3 wanted to investigate the 
geographical relations in more detail. Both participants changed the colour encoding of the data using multiple dimensions to examine various relations between the data. In most cases, this was followed by investigating the scatterplot to search for correlations between these different dimensions. Although the majority of the participants focused on using the dot map and filtering the parallel coordinates, Participant 1 and 4 were interested in the geo filtering capabilities of the software and tried to use it to investigate different aspects of the data.

During the use of the system, participants were able to generate hypotheses and used the system to either confirm or reject these hypotheses. The participants were able to identify interesting relations and explore various patterns of parallel coordinates interaction and geovisualization. For example, participants investigated the relationship between the amount of trash and population and concluded that there is no obvious relations between these factors.

All the participants in the group were enthusiastic about the software and its ability to explore different datasets. Even thought the field trials were conducted as a group study, every participant was able to use most of the main features of the system. As one participant expressed doubt about trusting the automatic outlier detection algorithm, this feature was not sufficiently used by all other participants.

\subsection{Discussion}

The goal of this research was to address challenges in high dimensional heterogeneous geotemporal data. The research focused on the analysis of environmental data as an example of such data. To evaluate the validity of the system, the evaluation hypotheses focused on the exploration and data analysis of the approach.

In the evaluation, field trial methodology was used to allow participants to use the 
system freely in a real world situation. The open end task allowed participants to use the system as they would in their daily work. The participants were targeted due to their knowledge in the dataset's domain. One of the main assumption of the evaluation was the participants' knowledge in the data. This is an important assumption as it ensured that the participants knew what the data means and were be able to use the system to make sense of the data. During their exploration of the data, the participants were able to confirm or reject their own hypotheses about the data from their knowledge of the domain.

As target users of the system are environmental researchers, selecting participant from this domain ensured that the reliability of participants' responses and the findings. They were able to provide a more realistic assessment of the system than non domain expert user. Additionally, a variety of methods were used to assess and analyze the data from the evaluations. As each method has its advantages, using mixed methods provided additional credibility for the findings of the evaluation. The results of all the method were consistent, which confirm and increase the validity of the findings.

A summary of the results of the evaluation is presented in Table 4.3 and it appears as if the GCPC either accept or supports all the hypotheses (i.e., H1, H2, H3a, H3b, and H3c). Table 4.3 also summarizes the supported hypothesis for each feature in the system. The parallel coordinates feature supports all the hypotheses except the output quality hypothesis, which had mixed responses. The misleading representation of missing values could be the reason for the mixed responses on output quality of the parallel coordinates. As some participants were experienced GIS users, their expectations for built-in spatial analysis is reflected in their responses to the geovisualization. A diversity of responses was reported in both the data exploration and output quality hypotheses for the geovisualization feature. In addition, since most participants were familiar with more sophisticated statistical analysis tools, they saw the micro-visualization of statistical information feature easy to use (i.e., 
Table 4.3: Summary of the evaluation results

\begin{tabular}{|l||c||c||c||c||c|}
\hline Features & H1 & H2 & H3a & H3b & H3c \\
\hline GCPC & Accepted & Supported & Accepted & Accepted & Supported \\
\hline \hline Parallel coordinates & Accepted & Supported & Accepted & Accepted & Mixed \\
\hline \hline Geovisualization & Supported & Mixed & Supported & Accepted & Supported \\
\hline \hline $\begin{array}{l}\text { Micro visualization of } \\
\text { statistical information }\end{array}$ & Supported & Mixed & Supported & Accepted & Mixed \\
\hline \hline $\begin{array}{l}\text { Investigative scatter- } \\
\text { plot }\end{array}$ & Mixed & Mixed & Supported & Accepted & Supported \\
\hline \hline Outlier detection & Mixed & Rejected & Mixed & Mixed & Rejected \\
\hline \hline
\end{tabular}

H3b) but not as useful (i.e., H3a). Additionally, participants found the investigative scatterplot useful as an analysis tool (i.e., H3) but not so as an exploration feature (i.e., H1). During the field trials, one participant expressed doubt about the result of the automatic outlier detection algorithm. As the field trials were conducted within a single focus group, this opinion is clearly reflected in the outlier detection results.

There are certain problems with the use of focus group evaluations. One of these is that participants' opinions could be biased by the statement of others, as it happened in the discussion of the outlier detection. However, the group discussion enabled the participants to understand the data more easily. As the analysis was conducted as a group activity, the group discussion was enriched by the various comments and suggestion from each participant. Additionally, to ensure that each participant was able to use the system sufficiently to evaluate it, each participant was asked to drive the system for part of the time.

The results of the evaluation indicate the benefits of the system for the exploration of environmental data. The evaluation provided evidence of the advantages of using the system for analyzing complex datasets, including high dimensional heterogeneous datasets. The evaluations showed that participants were positively engaged with the system and use to explore the data in various methods.

Explanation of the benefit of the system contributed to the growing body of knowledge 
the advantages of using the visual analytics approach and the visual exploration process. The use of the visual analytics approaches with a focus on interaction between users and the data allowed the system to provide useful and easy to use tools to analysts. Designing the system with an understanding of information visualization concepts and colour theories helped analysts to interpret the data more effectively. Researchers were able to explore the different aspects and subsets of the data.

Another valuable aspect of GCPC is the interactive investigative ability of the system. Participant were able to interactively investigate the relations between the different dimensions easily and quickly. During the field trials, participant acknowledged the flexibility of moving coordinates around, selecting dimension for the scatterplot, and changing the visual encoding. This flexibility allowed them to quickly explore and search for patterns and trends in the complex data.

The system supported participants in discovering information using a visual analytics process as proposed by Keim: "analyze first; show the important; zoom, filter, and analyze further; details on demand" [75]. The preliminary analysis of the data is performed by the system before displaying the data. Researchers were able to filter and zoom to explore the data in the main window. Further analysis was enabled by the system through the multiple coordinated interactions, dynamic encoding, and investigative scatter plot. Providing the details on demand helped researchers to get the information they needed to confirm or reject their hypotheses.

Another reason for the participants perception of the value of the system is the absence of similar software available to the researchers. GIS and other statistical analytical tool are examples of such tools, but these tools provide limited exploration capabilities for high dimensional heterogeneous data. With high dimensional data, GCPC provides an easy to use tool for data exploration while maintaining awareness of the geospatial aspect of the data. Participants saw the value of using the system as a preliminary analysis tool for high 
dimensional data. Part of the discussion focused on how to use the system on different datasets and how each researcher could use the system in their work. 


\section{Chapter 5}

\section{Conclusions and Future Work}

This Thesis presented Geo-Coordinated Parallel Coordinates, a geovisual analytics approach designed to support the exploration of high dimensional heterogeneous geotemporal data. A parallel coordinates plot tightly coupled with a geovisualization provides the main component of the system. The coordinated interaction between these two views allows the user to explore multiple aspects of the complex data. The parallel coordinates plot is equipped with multiple interactions to allow the user to analyze and understand complex patterns and trends in the high dimensional heterogeneous data. Additionally, the system contains a scatter plot view to support direct visual correlation analysis of multiple dimensions at the same time.

\subsection{Research Contributions}

The fundamental contribution of this research is the design, development, and study of a system that integrates multiple visualizations to support exploration and analysis of high dimensional heterogeneous geotemporal data. To address the challenges in exploring and analyzing the complex data, the research focused on developing an approach to address the challenges of visual exploration of high dimensional heterogeneous data, complex geotemporal data, and environmental data as an example of such data. The goal of the research 
was to investigate the design of various visual analytics methods to support the exploration and reasoning of such data. During this research, a prototype of a geovisual analytics system was implemented to study the approach for analyzing and exploring among complex environmental data.

The study confirmed previous research about the value of integrating multiple visualization and interaction methods [57, 89, 104]. With the goal of supporting the analysis of high dimensional heterogeneous geotemporal data, this research contribution provides evidence of the value of integrating the specific visualization and interaction methods that are used in the system. This fundamental contribution can be divided into the following: 1) the benefits of using of multiple visualization and interaction techniques to support exploration of the multiple aspects of the complex data, 2) enhancements to the parallel coordinates to support analysis of the high dimensional heterogeneous data, 3) the development and implementation ideas of GCPC prototype software, and 4) the study of the work and findings of the evaluation conducted by environmental researchers .

The first contribution of this work is the integration of multiple visual analytics techniques to address the research challenges. The presented approach supports exploration and reasoning about high dimensional heterogeneous geotemporal data. The system has merged multiple techniques to permit users to identify and explore the complex patterns and trends in the data. The tightly coupled parallel coordinates and geovisualization allow users to explore the high dimensional data while maintaining awareness of the geospatial aspect. The multiple coordinated interactions between the two views support users to detect and understand the different relationships within the data.

The research shows the value of supporting different data types in the analysis of environmental data. To support the analysis of a mix of qualitative, quantitative, and temporal data, the system identifies the type of the data and uses different visual encoding and analysis for these types. The use of different encoding for different types allowed for a deeper 
understanding of each dimension based on its type.

The second contribution of the system is the various enhancements to the parallel coordinates. These improvements provide users with better support for analyzing and understanding the high dimensional heterogeneous data. Micro-visualizations of the statistical information about the data are added to the parallel coordinates to allow users to easily observe the distribution of data. The proposed technique allows users to compare the various dimensions and get an overview of the data with a single glance.

Another improvement to the parallel coordinates is the integration of a high dimensional outlier detection in the system. The outlier detection permits users to detect data points different from the general distribution, which are difficult to identify in the parallel coordinates. In GCPC, the outlier detection recognizes and labels outlier points in a dataset. Users could investigate the outlier in more detail or remove them from the view if they are not interesting. Finally, the parallel coordinates is extended using multiple controls to filter and select the colour to encode the data.

The third contribution is the implementation of the GCPC prototype software. GCPC has been developed as a web based application using a three tier architecture. Several input scripts have been implemented to transform raw data to acceptable format of the system and store it in the database. The system has been deployed on a web server and several environmental datasets has been loaded to the database. Multiple D3 [23] plugins were utilized, modified, and extended to implement the main interface and the coordinated interactions.

The final contribution of this work is the outcome of evaluating the system domain experts using environmental datasets. Using a field trial methodology, the system was evaluated by multidisciplinary environmental scientists to explore and analyze multiple datasets. With the diverse number of factors, environmental researchers benefited from using the system to explore and examine the different aspects and relationships in the data. 
The system allowed them to explore the high dimensional data while remaining aware with the geospatial aspect of the data. The participants found that the system allowed them to easily communicate and explain the finds of their exploration and analysis. They reported the value, usefulness, and ease of use of the system in their initial exploration and analysis of the complex geotemporal data.

\subsection{Limitations}

Even though this Thesis demonstrated the benefit of GCPC, there are a number of limitations. One of these limitations is the lack of support for datasets with complex structures. Hierarchical data is an example of a complex data structure that is not supported by the GCPC. The use of parallel coordinates as one of the main views in the system limits the ability for displaying datapoints that have multiple hierarchical levels. This is a known drawback of using parallel coordinates, where each point in the data is represented using a single line intersecting the parallel coordinate at the appropriate location. One approach for supporting hierarchical information is to split the polyline to represent the sub-hierarchy, and then converging it again, but this adds ambiguity and result in significant visual clutter [49, 111].

Another data type that is limited by the system is the support for handling complex temporal structures. The system supports analysis of temporal information associated with data by allowing temporal fields to be analyzed in the parallel coordinates. However, GCPC lacks the ability to deal with temporal data that consists of multiple temporal values or temporal periods.

A third type of data that is hard to visualize within the parallel coordinates is relational data. Relational data where one factor consists of multiple values of another dimension creates a problem. Similar to hierarchical data, relational data represented by splitting 
datapoints at the coordinates adds complexity to the visualization. Missing values and fields with multiple values are additional examples of data types which GCPC does not handle well.

Another limitation of the system is the difficulty to detect clusters and similar patterns over multiple dimensions using the parallel coordinates. Even though we endeavoured to address some fundamental limitations of parallel coordinates in GCPC, it is still difficult to visually group and recognize similar patterns within the parallel coordinates. However, although these patterns are not automatically detected by GCPC, the investigative scatterplot could help users to recognize such patterns. By making a good choice of the dimensions used in the scatterplot, users will be able to identify patterns that are hard to see within the parallel coordinates.

A third limitation of the system is that the current implementation of the GCPC assumes the geospatial data are point data. The system does not support other types of geospatial data, such as area or line. Representing more geospatial types will generalize the approach and provide support for multiple datasets. Currently, the system replaces any polygon information with a point at the geometrical centre of mass of the polygon to represent geospatial region data. For example, a geospatial location of Prince Edward Island is represented as a single point located at the centre of the province.

Additionally, there are some limitations to the evaluation method used which limits the findings of the research. Due to the lack of similar baseline systems for environmental data, the study was not able to reliably compare the system to any other alternative. The small sample size and limited evaluation time did not allow for extensive evaluation of the system. This limited the collection of information on long term usefulness of the system in a real situations, or how the researchers will benefit from the system in their daily work. The evaluation was conducted as focus group evaluating the system and discussing the findings at the same time. This limited each user's ability to evaluate the ease of use of the 
system as they used it as a group. It allowed users to discuss their analysis at the same time, but it does not provide data from each user as it is more of a group analysis.

\subsection{Future Work}

Addressing the limitations of the system should include further enhancement to the parallel coordinates plot. One of the limitations is the support for more complex data types. Addressing this limitation will allow the system to support a wide range of datasets. To represent relational dimensions, the user should be able to identify the coordinates of these dimensions easily. New interactions should be added to support manipulation of the multiple values at these coordinates.

Another enhancement to the parallel coordinates is improving the outlier detection to provide a more precise mapping of qualitative and ordinal dimensions. The automatic anomaly detection algorithm could be supplemented with pattern matching algorithms such as clustering in order to allow similarities over the large number of dimensions to be isolated and evaluated. As users in the field trials were not confident of the outlier analysis, future study could investigate the use of an algorithm that could be easily explained to users or provide users with more control over the anomaly analysis.

With the proposed approach, only point geospatial data is supported. Future research should be carried out to add support for other geospatial features (i.e., area or line features). In terms of supporting various geospatial features, environmental researchers will benefit greatly from improving the current geospatial analysis. By adding support for area and line data type, more accurate geographical analysis could be conducted. Additionally, a larger number of environmental datasets will be supported by the system.

The analysis of geotemporal patterns was addressed by providing the user with multiple interactions between the parallel coordinates and geovisualization. The proposed approach 
allows user to explore the different geotemporal patterns. However, multiple temporal periods (i.e., events that occur in a substantial amount of time) are not supported by the system. Due to multiple values (i.e., start and end of each period) and granularity of the different temporal periods, such temporal data type adds complexity to the current representation. Further research might explore adding a separate view of the temporal data. Extending the system by adding another view for complex temporal data will enable users to analyze different types of temporal data and identify complex temporal patterns and trends.

Although the results of the field trials were positive in general, there is room for further evaluations. While the focus group study provided sufficient evidence of the value of the approach, it may be of value to investigate how individuals will use GCPC in their work. Additional studies to evaluate the approach by environmental researchers in a type of longitudinal studies will provide further understanding of the benefit and the usefulness of the approach in real-world setting. Further investigation of the approach using various datasets from multiple domains will illustrate the flexibility of GCPC.

The evaluation showed the benefit of using the system to explore the environmental data. As a group of researchers collaborated to examine the data, the benefit of supporting collaborative analysis was evident by observing their analysis activities. The collaboration allowed researchers to generate multiple hypotheses about the data at the same time, quickly identify various pattern, and share any gained insight about the data. Further research should be carried out to explore how to support collaborative analysis in a visual analytics system. Such investigation will focus on approaches to enable multiple analysts to view, explore, discuss, and analyze the same set of data. 


\section{References}

[1] mongodb.

[2] Vladimir Agafonkin. Leaflet. http://leafletjs.com/, 2010. Accessed: December 2014.

[3] Charu C. Aggarwal. Data Mining, The Textbook. Springer International Publishing, Cham, Switzerland, 2015.

[4] Sofia Ahlroth, Måns Nilsson, Göran Finnveden, Olof Hjelm, and Elisabeth Hochschorner. Weighting and valuation in selected environmental systems analysis tools - suggestions for further developments. Journal of Cleaner Production, 19(2-3):145-156, 2011.

[5] Jamal Mohamed Alsakran. Supporting interactive visual analytics for high dimensional data exploration. PhD. thesis, Kent State University, 2012.

[6] Jayanath Ananda and Gamini Herath. A critical review of multi-criteria decision making methods with special reference to forest management and planning. Ecological Economics, 68(10):2535-2548, 2009.

[7] Gennady Andrienko and Natalia Andrienko. Exploring spatial data with dominant attribute map and parallel coordinates. Computers, Environment and Urban Systems, 25(1):5-15, 2001. 
[8] Gennady Andrienko and Natalia Andrienko. Geovisual analytics for spatial decision support: Setting the research agenda. International Journal of Geographical Information Science, 21(8):839-857, 2007.

[9] Gennady Andrienko and Natalia Andrienko. Spatio-temporal aggregation for visual analysis of movements. In Proceedings of the IEEE Symposium on Visual Analytics Science and Technology, pages 51-58, 2008.

[10] Gennady Andrienko, Natalia Andrienko, Urska Demsar, Doris Dransch, Jason Dykes, Sara Irina Fabrikant, Mikael Jern, Menno-Jan Kraak, Heidrun Schumann, and Christian Tominski. Space, time and visual analytics. International Journal of Geographical Information Science, 24(10):1577-1600, 2010.

[11] Gennady Andrienko, Natalia Andrienko, Piotr Jankowski, Daniel Keim, Menno-Jan Kraak, Alan M. MacEachren, and Stefan Wrobel. Geovisual analytics for spatial decision support: Setting the research agenda. International Journal of Geographical Information Science, 21(8):839-857, 2007.

[12] Gennady Andrienko, Natalia Andrienko, Daniel Keim, Alan M. MacEachren, and Stefan Wrobel. Challenging problems of geospatial visual analytics. Journal of Visual Languages \& Computing, 22(4):251-256, 2011.

[13] Natalia Andrienko, Gennady Andrienko, and Peter Gatalsky. Exploratory spatiotemporal visualization: An analytical review. Journal of Visual Languages \& Computing, 14(6):503-541, 2003.

[14] Millennium Ecosystem Assessment. Ecosystems and human well-being: Synthesis. Island Press Washington, DC, 2005.

[15] Simon Bell. DPSIR=a problem structuring method? An exploration from the imagine approach. European Journal of Operational Research, 222(2):350-360, 2012. 
[16] Jacques Bertin. Semiology of Graphics. University of Wisconsin Press, Madison, WI, 1983.

[17] Enrico Bertini, Luigi Dell Aquila, Giuseppe Santucci, Sistemistica Universit, Roma La, and Via Salaria. SpringView: cooperation of Radviz and parallel coordinates for view optimization and clutter reduction. In Proceedings of the International Conference on Coordinated and Multiple Views in Exploratory Visualization, pages 22-29, 2005.

[18] Enrico Bertini, Andrada Tatu, and Daniel Keim. Quality metrics in high-dimensional data visualization: An overview and systemization. IEEE Transactions on Visualization and Computer Graphics, 17(6):2203-2212, 2011.

[19] Budhendra Bhaduri, Mallikarjun Shankar, Alexandre Sorokine, and Auroop Ganguly. Spatio-temporal visualization for environmental decision support. In Raffaele De Amicis, Radovan Stojanovic, and Giuseppe Conti, editors, GeoSpatial Visual Analytics, NATO Science for Peace and Security Series C: Environmental Security, pages 331-341. Springer Netherlands, 2009.

[20] Anthony E. Boardman. Cost-benefit analysis. In James D. Wright, editor, International Encyclopedia of the Social \& Behavioral Sciences, pages 47-54. Elsevier, Oxford, second edition edition, 2015.

[21] Thomas Boogaerts, Georgios A Pavlopoulos, Jan Aerts, and Joos Vandewalle. Visualizing high dimensional datasets using parallel coordinates: Application to gene prioritization. In Proceeding of the IEEE International Conference on Bioinformatics and Bioengineering, pages 11-13, 2012.

[22] Rita Borgo, Johannes Kehrer, David H. S. Chung, Eamonn Maguire, Robert S. 
Laramee, Helwig Hauser, Matthew Ward, and Min Chen. Glyph-based visualization: Foundations, design guidelines, techniques and applications. In Eurographics State of the Art Reports, pages 39-63, 2013.

[23] Michael Bostock, Vadim Ogievetsky, and Jeffrey Heer. D3 data-driven documents. IEEE Transactions on Visualization and Computer Graphics, 17(12):2301-2309, 2011.

[24] Cynthia A. Brewer, Geoffrey W. Hatchard, and Mark A. Harrower. ColorBrewer in print: A catalog of color schemes for maps. Cartography and Geographic Information Science, 30(1):5-32, 2003.

[25] Bertjan Broeksema, Thomas Baudel, Arthur G. Telea, and Paolo Crisafulli. Decision exploration lab: A visual analytics solution for decision management. IEEE Transactions on Visualization and Computer Graphics, 19(12):1972-1981, 2013.

[26] Jonathan Von Brünken, Michael E. Houle, and Arthur Zimek. Intrinsic dimensional outlier detection in high dimensional data. Technical report, National Institute of Informatics, Tokyo, 2015.

[27] Sheelagh Carpendale. Evaluating information visualizations. In Andreas Kerren, John T. Stasko, Jean-Daniel Fekete, and Chris North, editors, Information Visualization: Human-Centered Issues and Perspectives, pages 19-45. Springer-Verlag, Berlin, Heidelberg, 2008.

[28] Daniel B. Carr, Anthony R. Olsen, and Denis White. Hexagon mosaic maps for displaying univariate and bivariate geographical data. Cartography \& Geographical Information Systems, 19(4):228-236, 1992.

[29] Kai Chang. Parallel coordinates toolkit. https://syntagmatic.github. io/parallel-coordinates/, 2012. Accessed: December 2014. 
[30] Daniel Cheng, Peter Schretlen, Nathan Kronenfeld, Neil Bozowsky, and William Wright. Tile based visual analytics for twitter big data exploratory analysis. In Proceedings of the IEEE International Conference on Big Data, pages 2-4, 2013.

[31] So-Min Cheong. A new direction in coastal management. Marine Policy, 32(6):1090-1093, Nov 2008.

[32] Yi-Chang Chiang, Fen-Fang Tsai, Han-Pi Chang, Chi-Farn Chen, and Yi-Chia Huang. Adaptive society in a changing environment: Insight into the social resilience of a rural region of Taiwan. Land Use Policy, 36:510-521, 2014.

[33] Jong Youl Choi, Seung-Hee Bae, Judy Qiu, Geoffrey Fox, Bin Chen, and David Wild. Browsing large scale cheminformatics data with dimension reduction. In Proceedings of the ACM International Symposium on High Performance Distributed Computing, pages 503-507, 2010.

[34] Jong Youl Choi, Seung-Hee Bae, Xiaohong Qiu, and Geoffrey Fox. High performance dimension reduction and visualization for large high-dimensional data analysis. In Proceedings of the IEEE/ACM International Conference on Cluster, Cloud and Grid Computing, pages 331-340, 2010.

[35] David HS. Chung, Philip A. Legg, Matthew L. Parry, Rhodri Bown, Iwan W. Griffiths, Robert S. Laramee, and Min Chen. Glyph sorting: Interactive visualization for multi-dimensional data. Information Visualization, 14(1):79-90, 2015.

[36] John W. Creswell. Research Design: Qualitative, Quantitative, and Mixed Methods Approaches. Sage Publications, Inc, 4th edition, 2013.

[37] Mary Ann Curran. Environmental life-cycle assessment. The International Journal of Life Cycle Assessment, 1(3):179-179, 1996. 
[38] Clodoveu A. Davis, Frederico T. Fonseca, and Karla A. V. Borges. A flexible addressing system for approximate geocoding. In Proceedings of GeoInfo, pages 1-8, 2003.

[39] Luís Dias, Nelson Silva, Tiago Cardoso, and Manuel J. Fonseca. Understanding city dynamics through spatio-temporal visualization. In Proceedings of the Eurographics Workshop on Urban Data Modelling and Visualisation, pages 37-40, 2013.

[40] David L. Donoho. High-dimensional data analysis: The curses and blessings of dimensionality. In Proceedings of the AMS Conference on Math Challenges of the 21st Century, pages 1-33, 2000.

[41] Marian Dörk, Sheelagh Carpendale, Christopher Collins, and Carey Williamson. VisGets: Coordinated visualizations for web-based information exploration and discovery. IEEE Transactions on Visualization and Computer Graphics, 14(6):1205$1212,2008$.

[42] EEA. Environmental indicators: Typology and overview. Technical Report 25, European Environment Agency, Copenhagen, 1999.

[43] Maha El Meseery and Orland Hoeber. Geo-Coordinated Parallel Coordinates (GCPC): A case study of environmental data analysis. In Proceedings of Discovery Science, Lecture Notes in Computer Science, pages 63-77. Springer International Publishing, 2015.

[44] René A. Enguehard, Orland Hoeber, and Rodolphe Devillers. Interactive exploration of movement data: A case study of geovisual analytics for fishing vessel analysis. Information Visualization, 12(1):65-84, 2012.

[45] Jianqing Fan, Fang Han, and Han Liu. Challenges of big data analysis. National Science Review, 1(2):293-314, 2014. 
[46] Göran Finnveden and Åsa Moberg. Environmental systems analysis tools - an overview. Journal of Cleaner Production, 13(12):1165-1173, 2005.

[47] David Flanagan. JavaScript: The Definitive Guide. O'Reilly \& Associates, Inc., Sebastopol, CA, USA, 6th edition, 2011.

[48] The Apache Software Foundation. Apache HTTP server project. https:// httpd.apache.org/, 1997. Accessed: December 2015.

[49] Thomas R. Gabriel, A. Simona Pintilie, and Michael R. Berthold. Exploring hierarchical rule systems in parallel coordinates. In Proceedings of the International Symposium on Intelligent Data Analysis, pages 97-108, 2005.

[50] Mark Gahegan, Masahiro Takatsuka, Mike Wheeler, and Frank Hardisty. Introducing Geo VISTA Studio: An integrated suite of visualization and computational methods for exploration and knowledge construction in geography. Computers, Environment and Urban Systems, 26(4):267-292, 2002.

[51] Maria Gasalla, Silvia Salas, Patrick McConney, Rodrigo P. Medeiros, Ratana Chuenpagdee, and Beatrice Frank. Report on enhancing stewardship in small-scale fisheries through ecosystem approaches and other means. Technical report, Too Big to Ignore Latin America and the Caribbean Joint Workshop with Working Group 4, Curitiba, Brazil. TBTI, Canada, August 2013.

[52] Peter Gatalsky, Natalia Andrienko, and Gennady Andrienko. Interactive analysis of event data using space-time cube. In Proceedings of the International Conference on Information Visualisation, pages 145-152, 2004.

[53] Richard L. S. F. George, Peter E. Robins, Alan G. Davies, Panagiotis D. Ritsos, and Jonathan C. Roberts. Interactive visual analytics of hydrodynamic flux for the coastal zone. Environmental Earth Sciences, 72(10):3753-3766, 2014. 
[54] Alyssa A. Goodman. Principles of high-dimensional data visualization in astronomy. Astronomische Nachrichten, 333(5-6):505-514, 2012.

[55] Georges Grinstein, Marjan Trutschl, and Urška Cvek. High-dimensional visualizations. In Proceedings of the Visual Data Mining Workshop, pages 1-14, 2001.

[56] PHP Group. PHP: hypertext preprocessor. http://php . net/, 2001. Accessed: December 2015.

[57] Diansheng Guo, Jin Chen, Alan M. MacEachren, and Ke Liao. A visualization system for space-time and multivariate patterns (VIS-STAMP). IEEE Transactions on Visualization and Computer Graphics, 12(6):1461-1474, 2006.

[58] Helwig Hauser, Florian Ledermann, and Helmut Doleisch. Angular brushing of extended parallel coordinates. In Proceedings of the IEEE Symposium on Information Visualization, pages 127-134, 2002.

[59] Julian Heinrich, John Stasko, and Daniel Weiskopf. The parallel coordinates matrix. In Procdeeing of Eurographics Conference on Visualization, pages 37-41, 2012.

[60] Julian Heinrich and Daniel Weiskopf. State of the art of parallel coordinates. In Proceedings of Eurographics - State of the Art Reports, pages 95-116, 2013.

[61] Ewald Hering. Outlines Of A Theory Of The Light Sense. Harvard University Press, 1964.

[62] Quan Van Ho, Patrik Lundblad, Tobias Åström, and Mikael Jern. A web-enabled visualization toolkit for geovisual analytics. In Proceedings of SPIE Conference on Visualization and Data Analysis, volume 7868, pages 22-42, 2011. 
[63] Orland Hoeber. User evaluation methods for visual web search interfaces. In Proceedings of the International Conference Information Visualisation, pages 139-145, 2009.

[64] Orland Hoeber, Larena Hoeber, Laura Wood, Ryan Snelgrove, Isabella Hugel, and Dayne Wagner. Visual twitter analytics: Exploring fan and organizer sentiment during Le Tour de France. In Proceedings of the VIS, Workshop on Sports Data Visualization, pages 1-7, 2013.

[65] Orland Hoeber, Garnett Wilson, Simon Harding, René Enguehard, and Rodolphe Devillers. Exploring geo-temporal differences using GTdiff. In Proceedings of the IEEE Pacific Visualization Symposium, pages 139-146, 2011.

[66] Patrick Hoffman, Georges Grinstein, and David Pinkney. Dimensional anchors: A graphic primitive for multidimensional multivariate information visualizations. In Proceedings of the Workshop on New Paradigms in Information Visualization and Manipulation in Conjunction with the ACM International Conference on Information and Knowledge Management, pages 9-16, 1999.

[67] Mattias Höjer, Sofia Ahlroth, Karl-Henrik Dreborg, Tomas Ekvall, Göran Finnveden, Olof Hjelm, Elisabeth Hochschorner, Måns Nilsson, and Viveka Palm. Scenarios in selected tools for environmental systems analysis. Journal of Cleaner Production, 16(18):1958-1970, 2008.

[68] Jean-François Im, Michael J. McGuffin, and Rock Leung. GPLOM: The generalized plot matrix for visualizing multidimensional multivariate data. IEEE Transactions on Visualization and Computer Graphics, 19(12):2606-14, 2013.

[69] Alfred Inselberg. The plane with parallel coordinates. The Visual Computer, 1(2):69-91, 1985. 
[70] Stefan Jänicke, Christian Heine, and Gerik Scheuermann. GeoTemCo: Comparative visualization of geospatial-temporal data with clutter removal based on dynamic Delaunay triangulations. In Proceedings of the International Conference on Information Visualization Theory and Applications, pages 160-175, 2013.

[71] Jimmy Johansson. Efficient Information Visualization of Multivariate and TimeVarying Data. PhD thesis, Linköping University Institute of Technology, 2008.

[72] Ian T. Jolliffe. Principal Component Analysis. Springer Verlag, 1986.

[73] Rassadarie Kanjanabose. An empirical study on parallel coordinates and scatter plots. Master's thesis, Green Templeton College, University of Oxford, 2014.

[74] Joseph R. Kasprzyk, Shanthi Nataraj, Patrick M. Reed, and Robert J. Lempert. Many objective robust decision making for complex environmental systems undergoing change. Environmental Modelling \& Software, 42:55-71, 2013.

[75] Daniel Keim, Gennady Andrienko, Jean-Daniel Fekete, Carsten Görg, Jörn Kohlhammer, and Guy Melançon. Visual analytics: Definition, process, and challenges. In Andreas Kerren, John T. Stasko, Jean-Daniel Fekete, and Chris North, editors, Information Visualization: Human-Centered Issues and Perspectives, pages 154-175. Springer-Verlag, 2008.

[76] Daniel A. Keim, Jörn Kohlhammer, Florian Mansmann, Thorsten May, and Franz Wanner. Solving problems with visual analytics. In Jörn Kohlhammer, Daniel Keim, Geoffrey Ellis, and Florian Mansmann, editors, Mastering the Information Age, volume 7, pages 117-120. Eurographics Association, Goslar, Germany, 2011.

[77] Kurt Koffka. Principles of Gestalt Psychology. Harcourt Brace and Company, 1935. 
[78] Martin Komenda and Daniel Schwarz. Visual analytics in environmental research: A survey on challenges, methods and available tools. In Proceedings of the International Symposium on Environmental Software Systems, pages 618-629, 2013.

[79] Robert Kosara, Fabian Bendix, and Helwig Hauser. Parallel sets: Interactive exploration and visual analysis of categorical data. IEEE Transactions on Visualization and Computer Graphics, 12(4):558-68, 2006.

[80] Menno-Jan Kraak. The space-time cube revisited from a geovisualization perspective. In Proceedings of the International Cartographic Conference, pages 19881995, 2003.

[81] Hans-Peter Kriegel, Matthias Schubert, and Arthur Zimek. Angle-based outlier detection in high-dimensional data. In Proceedings of the ACM SIGKDD International Conference on Knowledge Discovery and Data Mining, pages 444-452, 2008.

[82] Marcin Kulawiak, Aristides Prospathopoulos, L. Perivoliotis, M. Euba, Sotiris Kioroglou, and Andrzej Stepnowski. Interactive visualization of marine pollution monitoring and forecasting data via a Web-based GIS. Computers \& Geosciences, 36(8):1069-1080, 2010.

[83] Heidi Lam, Enrico Bertini, Petra Isenberg, Catherine Plaisant, and Sheelagh Carpendale. Empirical studies in information visualization: Seven scenarios. IEEE Transactions on Visualization and Computer Graphics, 18(9):1520-1536, 2012.

[84] Jing Li, Jean-Bernard Martens, and Jarke J. van Wijk. Judging correlation from scatterplots and parallel coordinate plots. Information Visualization, 9(1):13-30, 2008 .

[85] Xia Li. New methods of visualization of multivariable spatio- temporal data: Pcp- 
time-cube and multivariable-time-cube. Master's thesis, International Institute for Geo-information Science and Earth Observation, 2005.

[86] Patrik Lundblad, Mikael Jern, and Camilla Forsell. Voyage analysis applied to geovisual analytics. In Proceedings of the International Conference Information Visualisation, pages 381-388, 2008.

[87] Wei Luo and Alan M. MacEachren. Geo-social visual analytics. Journal of Spatial Information Science, 8(8):27-66, 2014.

[88] Alan M. MacEachren, Anuj Jaiswal, Anthony C. Robinson, Scott Pezanowski, Alexander Savelyev, Prasenjit Mitra, Xiao Zhang, and Justine Blanford. Senseplace2: Geotwitter analytics support for situational awareness. In Proceedings of the IEEE Conference on Visual Analytics Science and Technology, pages 181-190, 2011.

[89] Adelina Agneli Malunda. Interactive dynamic visualization environment linking animated map and animated parallel coordinate plot for monitoring geospatial dynamics. Master's thesis, International Institute for Geo-Information Science and Earth Observation, 2005.

[90] Adam Marcus, Michael S. Bernstein, Osama Badar, David R. Karger, Samuel Madden, and Robert C. Miller. TwitInfo: aggregating and visualizing microblogs for event exploration. In Proceedings of the Annual Conference on Human Factors in Computing Systems, pages 227-236, 2011.

[91] Allen R. Martin and Matthew O. Ward. High dimensional brushing for interactive exploration of multivariate data. In Proceedings of the IEEE Conference on Visualization, pages 271-278, 1995. 
[92] Riccardo Mazza and Alessandra Berre. Focus group methodology for evaluating information visualization techniques and tools. In Proceedings of the International Conference Information Visualization, pages 74-80, 2007.

[93] Kathy T. Mullen. The contrast sensitivity of human colour vision to red-green and blue-yellow chromatic gratings. The Journal of physiology, 359:381-400, 1985.

[94] Julia Eunju Nam and Klaus Mueller. TripAdvisorN-D: A tourism-inspired highdimensional space exploration framework with overview and detail. IEEE transactions on visualization and computer graphics, 19(2):291-305, 2012.

[95] Alice Newton and Juergen Weichselgartner. Hotspots of coastal vulnerability: A DPSIR analysis to find societal pathways and responses. Estuarine, Coastal and Shelf Science, 140(1):123-133, 2014.

[96] Matej Novotný and Helwig Hauser. Outlier-preserving focus+context visualization in parallel coordinates. IEEE Transactions on Visualization and Computer Graphics, 12(5):893-900, 2006.

[97] Gregorio Palmas, Myroslav Bachynskyi, Antti Oulasvirta, Hans Peter Seidel, and Tino Weinkauf. An edge-bundling layout for interactive parallel coordinates. In Proceedings of the IEEE Pacific Visualization Symposium, pages 57-64, 2014.

[98] Maria Rosário Partidário. Strategic environmental assessment: Key issues emerging from recent practice. Environmental Impact Assessment Review, 16(1):31 - 55, 1996.

[99] Richard Pollnac and Tarsila Seara. Factors influencing success of marine protected areas in the visayas, philippines as related to increasing protected area coverage. Environmental Management, 47(4):584-592, 2011. 
[100] Richard B. Pollnac, Brian R. Crawford, and Maharlina L.G. Gorospe. Discovering factors that influence the success of community-based marine protected areas in the visayas, philippines. Ocean \& Coastal Management, 44(11-12):683-710, 2001.

[101] Anil Ramakrishna, Yu-Han Chang, and Rajiv Maheswaran. An interactive web based spatio-temporal visualization system. In Proceedings of the International Symposium on Advances in Visual Computing, pages 673-680, 2013.

[102] Naomi B. Robbins, Richard M. Heiberger, Christine Court, and Speakman Hall. Plotting likert and other rating scales. Joint Statistical Meetings, (October 2005):1058-1066, 2011.

[103] Jonathan C. Roberts. State of the art: Coordinated \& multiple views in exploratory visualization. In Proceedings of the International Conference on Coordinated and Multiple Views in Exploratory Visualization, pages 61-71, 2007.

[104] Jonathan C. Roberts. Coordinated multiple views for exploratory geovisualization. In Martin Dodge, Mary McDerby, and Martin Turner, editors, Geographic Visualization, pages 25-48. John Wiley \& Sons, Ltd, 2008.

[105] Juan M. Sánchez-Lozano, Jerónimo Teruel-Solano, Pedro L. Soto-Elvira, and Maria Socorro García-Cascales. Geographical information systems (GIS) and multicriteria decision making (MCDM) methods for the evaluation of solar farms locations: Case study in south-eastern Spain. Renewable and Sustainable Energy Reviews, 24:544-556, 2013.

[106] Maximilian Scherr. Multiple and coordinated views in information visualization. In Trends in Information Visualization. Technical report, Ludwig-MaximiliansUniversity in Munich, 2008. 
[107] J. Scholtz. Beyond usability: Evaluation aspects of visual analytic environments. In Proceedings of the IEEE Symposium On Visual Analytics Science And Technology, pages 145-150, 2006.

[108] Heidrun Schumann and Christian Tominski. Analytical, visual and interactive concepts for geo-visual analytics. Journal of Visual Languages \& Computing, 22(4):257-267, 2011.

[109] Yong Shi. Detecting clusters and outliers for multi-dimensional data. In Proceedings of the International Conference on Multimedia and Ubiquitous Engineering, pages 429-432, 2008.

[110] Harri Siirtola. Combining parallel coordinates with the reorderable matrix. In Proceedings International Conference on Coordinated and Multiple Views in Exploratory Visualization, pages 63-74, 2003.

[111] Harri Siirtola and Kari-Jouko Räihä. Interacting with parallel coordinates. Interacting with Computers, 18(6):1278-1309, 2006.

[112] Mike Sips, Patrick Kothur, and Andrea Unger. A visual analytics approach to multiscale exploration of environmental time series. IEEE Transactions on Visualization and Computer Graphics, 18(12):2899-2907, 2012.

[113] Ruth D. Swetnam, Brendan Fisher, Boniphace P. Mbilinyi, Pantaleo K. T. Munishi, S. Willcock, Taylor Ricketts, Shadrack Mwakalila, Andrew Balmford, Neil D. Burgess, Andrew R. Marshall, and Simon L. Lewis. Mapping socio-economic scenarios of land cover change: a GIS method to enable ecosystem service modelling. Journal of Environmental Management, 92(3):563-74, 2011. 
[114] Laura Tateosian, Sidharth Thakur, Eric Hardin, and Helena Mitasova. Visualizing coastal spatial-temporal dynamics. In Proceedings of Working with Uncertainty Workshop, pages 2-3, 2011.

[115] Dennis Thom, Harald Bosch, and Steffen Koch. Spatiotemporal anomaly detection through visual analysis of geolocated twitter messages. In Proceedings of the IEEE Pacific Visualization Symposium, pages 41-48, 2012.

[116] James J. Thomas and Kristin A. Cook. A visual analytics agenda. IEEE Computer Graphics and Applications, 26(1):10-13, 2006.

[117] Brain M. Tomaszewski and Anthony C. Robinson. Geovisual analytics and crisis management. In Proceedings of the International Information Systems for Crisis Response and Management (ISCRAM) Conference, pages 173-179, 2007.

[118] Edward Tufte. Envisioning Information. Graphics Press, Cheshire, CT, USA, 1990.

[119] Edward Tufte. Visual Explanations. Graphics Press, Cheshire, CT, USA, 1997.

[120] Edward R. Tufte. The Visual Display of Quantitative Information. Graphics Press, Cheshire, CT, USA, 2nd edition, 2001.

[121] Vasilis D. Valavanis, Graham J. Pierce, Alain F. Zuur, Andreas Palialexis, Anatoly Saveliev, Isidora Katara, and Jianjun Wang. Modelling of essential fish habitat based on remote sensing, spatial analysis and GIS. Hydrobiologia, 612(1):5-20, 2008.

[122] Jarke J. Van Wijk. The value of visualization. In Proceedings of the IEEE Visualization, pages 79-86, 2005.

[123] Viswanath Venkatesh and Fred D. Davis. A theoretical extension of the technology acceptance model: Four longitudinal field studies. Management Science, 46(2):186204, 2000. 
[124] Tatiana Von Landesberger, Sebastian Bremm, Natalia Andrienko, Gennady Andrienko, and Maria Tekusova. Visual analytics methods for categoric spatiotemporal data. In Proceedings of the IEEE Conference on Visual Analytics Science and Technology, pages 183-192, 2012.

[125] Rick Walker, Philip A. Legg, Serban Pop, Zhao Geng, Robert S. Laramee, and Jonathan C. Roberts. Force-directed parallel coordinates. In Proceedings of the International Conference on Information Visualisation, pages 36-44, 2013.

[126] Matthew Ward, Georges Grinstein, and Daniel Keim. Interactive Data Visualization: Foundations, Techniques, and Applications. A. K. Peters, Ltd., Natick, MA, USA, 2010.

[127] Colin Ware. Information Visualization: Perception for Design. Morgan Kaufmann Publishers Inc., Waltham, MA, USA, 3rd edition, 2013.

[128] Leland Wilkinson, Anushka Anand, and Robert Grossman. High-dimensional visual analytics: Interactive exploration guided by pairwise views of point distributions. IEEE Transactions on Visualization and Computer Graphics, 12(6):13631372, 2006.

[129] Ningchuan Xiao, David A. Bennett, and Marc P. Armstrong. Interactive evolutionary approaches to multiobjective spatial decision making: A synthetic review. Computers, Environment and Urban Systems, 31(3):232-252, 2007.

[130] Jianting Zhang and Le Gruenwald. Embedding and extending GIS for exploratory analysis of large-scale species distribution data. In Proceedings of the ACM SIGSPATIAL International Conference on Advances in Geographic Information Systems, pages 28:1-28:10, 2008. 
[131] Tao Zhang, Qi Liao, Lei Shi, and Weishan Dong. Spatiotemporal anomaly visualization for large-scale dynamic networks. In Proceedings of the Visualization Workshop, pages 1-2, 2013.

[132] Chen Zhong, Tao Wang, Wei Zeng, and Stefan Müller Arisona. Spatiotemporal visualisation: A survey and outlook. In Stefan Müller Arisona, Gideon Aschwanden, Jan Halatsch, and Peter Wonka, editors, Digital Urban Modeling and Simulation, volume 242, pages 299-317. Springer Berlin Heidelberg, 2012.

[133] Hong Zhou, Weiwei Cui, Huamin Qu, Yingcai Wu, Xiaoru Yuan, and Wei Zhuo. Splatting the lines in parallel coordinates. Computer Graphics Forum, 28(3):759$766,2009$.

[134] Hong Zhou, Xiaoru Yuan, Huamin Qu, Weiwei Cui, and Baoquan Chen. Visual clustering in parallel coordinates. Computer Graphics Forum, 27(3):1047-1054, 2008. 


\section{Appendix A}

\section{Research Ethics Board Approvals}

Note: The signatures were intentionally blurred from the Research Ethics Board approval memos. 


\section{University \\ of Regina \\ Research Ethics Board \\ Certificate of Approval}

PRINCIPAL INVESTIGATOR

Maha El Messery

SUPERVISOR

Dr. Orland Hoeber

FUNDER(S)

Supervisor is a Co-Applicant on a SSHRC Grant

TITLE

Field Trials of Geo-Coordinated Parallel Coordinates

APPROVAL OF

Application for Behavioural Research Ethics Review

Recruitment Email \#1 - Site 1: TBTI

Recruitment Email \#2 - Site 2: SYSENC

Email \#3 - Conformation of Participation

Consent Form

Pre-Task Questionnaire

Post-Task Questionnaire
DEPARTMENT

Computer Science
REB\#

2015-094
APPROVED ON

July 13, 2015
RENEWAL DATE

July 13, 2016

Full Board Meeting

Delegated Review

$\triangle$

CERTIFICATION

The University of Regina Research Ethics Board has reviewed the above-named research project. The proposal was found to be acceptable on ethical grounds. The principal investigator has the responsibility for any other administrative or regulatory approvals that may pertain to this research project, and for ensuring that the authorized research is carried out according to the conditions outlined in the original protocol submitted for ethics review. This Certificate of Approval is valid for the above time period provided there is no change in experimental protocol, consent process or documents.

Any significant changes to your proposed method, or your consent and recruitment procedures should be reported to the Chair for Research Ethics Board consideration in advance of its implementation.

\section{ONGOING REVIEW REQUIREMENTS}

In order to receive annual renewal, a renewal report must be submitted to the REB Chair for Board consideration within one month of the current expiry date each year the study remains open, and upon study completion. Please refer to the following website for further instructions: http://www.ureqina.ca/research/for-facultystaff/ethics-compliance/human/forms1/ethics-forms.html

Dr. David Senkow, Acting Chair

University of Regina

Research Ethics Board 


\section{University ofRegina}

PRINCIPAL INVESTIGATOR

Maha El Meseery

SUPERVISOR

Dr. Orland Hoeber

TITLE

Field Trials of Geo-Coordinated Parallel Coordinates

AMENDMENT APPROVAL

OF

Consent Forms

Recruitment Materials
Research Ethics Board

Certificate of Amendment Approval
DEPARTMENT

Computer Science
REB\#

2015-094

Full Board Meeting

Delegated Review $\bigotimes$

ORIGINAL DATE of NEXT RENEWAL DATE APPROVAL

July 13, 2015

July 13, 2016
Date of Amendment Approval

September 15, 2015

\section{AMENDMENT CERTIFICATION}

The University of Regina Research Ethics Board has reviewed the changes to the above-named research project as outlined in your memo dated September 15, 2015, and they are approved.

\section{ONGOING REVIEW REQUIREMENTS}

In order to receive annual renewal, a status report must be submitted to the REB Chair for Board consideration within one month of the current expiry date each year the study remains open, and upon study completion. Please refer to the following website for further instructions:

http://www.uregina.ca/research/for-faculty-staff/ethics-compliance/human/forms 1/ethics-forms.html

Dr. Larena Hoeber, Chair

University of Regina

Research Ethics Board 
Appendix B

\section{Study Documents}




\section{University \\ of Regina}

DEPARTMENT OF COMPUTER SCIENCE

Participant Consent Form

\section{Project Title: Field Trials for Geo-Coordinated Parallel Coordinates (SESYNC Focus Group)}

Researcher: Maha El Meseery

M.Sc. Student

Department of Computer Science

University of Regina

elmeseem@uregina.ca

306-515-1357

Supervisor: $\quad$ Dr. Orland Hoeber

Associate Professor

Department of Computer Science

University of Regina

306-585-4590

orland.hoeber@,uregina.ca

\section{Purpose(s) and Objective(s) of the Research:}

- This focus group will be conducted to evaluate the usefulness and effectiveness in supporting exploration and analysis of environmental data using Geo-Coordinated Parallel Coordinates (GCPC).

\section{Procedures:}

- An electronic copy of this consent will be emailed to all participants in this focus group when they agree to participate in this study. At that time, the specific date and time for the focus group session will be arranged.

- At the beginning of the study, you and all participants will be given the two-printed copies of this consent form. You will be asked to read and review the document, and will be encouraged to ask any questions you might have about the study procedures, your role within the study, and the goal of the study.

- Once you are satisfied with the information you have received about the study and you agree to continue participation in the focus group, you will be asked to sign the two copies of the consent form. The researcher will sign and date both copies, keep one copy with the study documents, and give you one copy for your records.

- Before using the system, the researcher will ask all participants to answer a pre-task questionnaire to get more information about their experiences with coastal environment domain, data visualization, geographic information systems, and similar software systems.

- The researcher will then introduce GCPC to the group and give a short demonstration of the key features of the system and how to use them to analyze environmental data. Participants are encouraged to ask any question about anything in the system or try to operate the system by themselves.

- The researcher will provide the group with an environmental dataset related to their research domain. Participants will be given the opportunity to take turns using the software to explore among the data and discuss what has been found. This will be followed with a similar group analysis of a second dataset with a larger number of dimensions.

- During the use of GCPC, the discussion and use of the system will be video and audio recorded. The camera will be positioned behind the group, so that the participants will not be readily identified from the video and the focus of the video will be on the computer screen. This data will 
be collected so that the discussion of the system features and the interaction with the system can be analyzed at a later date. The researcher will also act as a facilitator for the discussion and will be responsible for taking notes about the main ideas expressed by the group.

- After the group finishes with the two analysis activities, you will be asked to complete a questionnaire about the system and its key features. The questions will focus on measuring your impression of the usefulness, ease of use, and exploration and analysis ability of the software.

- In the final step of the study, the researcher will initiate a group discussion about the software. You will be given an opportunity to elaborate on your impressions of the positive or negative features of the system, how you think the system might support analysis of environmental data, and how you might be able to integrate the system their work.

- It is expected that entire study will take between 60 - 120 minutes, depending on how long the group chooses to use the software to analyze data provided and how long the group discussion takes.

\section{Funded by:}

This research is funded as part of the Too Big To Ignore (TBTI) project with grant from the Social Sciences and Humanities Research Council of Canada (SSHRC).

\section{Potential Risks:}

- There are no known or anticipated risks to you or any participant in the group by participating in this research beyond normal work activities of using a computer, filling out paperwork, and answering questions.

\section{Potential Benefits:}

- Your participation in the study will help determine the value of GCPC in supporting environmental data analysis. We will be able to determine which feature of GCPC are helpful in analyzing environmental data and which features require modification or refinement.

- The findings of the study will provide evidence of the general value of visual analytics approaches to environmental data analysis and research.

- This study may allow you to discover interesting features and aspect of environmental data in your domain. It will permit you to experience the power of combining visualization and automatic analysis to explore complex heterogeneous data.

\section{Anonymity}

- Due to the natural of group activities, other participants in the focus group will know your identity and your responses during the analysis and group discussions. However, beyond the participants of this study you will not be identified or linked to the result.

- Other than signing this consent form, you will not be required to identify yourself on any other documents.

- All information that identifies you as a participant in this study (emails to coordinate participation date and time, this consent form) will be kept completely separate from the collected data, and no connection between the two will be possible.

- The details of your participation will not be explicitly shared with anyone outside of the research team (the student researcher/investigator and her supervisor).

- You will be asked to respect the privacy of the other group members, and to not disclose any personal opinions of other members of the group activity. However, it is important to understand that other people in the group with you may not keep all information private and confidential.

\section{Confidentiality:}

- All raw data collected in the course of this study will be kept confidential, and will only be used by the researcher and the researcher's supervisor. 
- All raw data will be transported and stored in a secure manner (carry on luggage during transportation, a locked filing cabinet in a locked faculty office at the University of Regina, and on password protected computers/data archives).

- Raw data will be kept for five years after the conclusion of the study, and will then be discarded in accordance with university policy for secure document and file destruction.

- Individual participants will be not identified or linked to the result; anonymous opinions and quotations will be used instead.

- Aggregate data from the questionnaires, anonymous quotations from the discussions, and observations from the video recordings will be used in the publication and reporting of the study.

\section{Right to Withdraw:}

- Whether you choose to participate or not will have no effect on your relation with the researcher or the supervisor or how you will be treated.

- During the course of the focus group session, you may choose to not answer any question or perform any activity that makes you uncomfortable.

- You may choose to withdraw from the study for any reason, at any time before or during the focus group without explanation or penalty of any sort.

- Should you choose to withdraw, you may exit the study room and we will continue the group activities without you. However, we will not be able to remove any data we have collected from you because of the difficulty of isolating your activities from those of other participants in the group.

\section{Follow up:}

- After the conclusion of the study, the researcher will post all publications based on the study on the project websites and supervisor webpage.

- To obtain the results from the study, please visit the project website: http://demo.vista.uregina.ca/GCPC/publications

\section{Questions or Concerns:}

- If you have any questions about this project, feel free to contact the researcher or supervisor using the information at the top of the first page of this consent form.

- This project has been approved on ethical grounds by the UofR Research Ethics Board on (13 July 2015).Any questions regarding your rights as a participant may be addressed to the committee at (306-585-4775 or research.ethics@uregina.ca). Out of town participants may call collect.

\section{Consent:}

My signature below indicates that I have read and understand the description provided. I have had an opportunity to ask questions and my questions have been answered. I consent to participate in the research project.

Name of Participant

Researcher's Signature

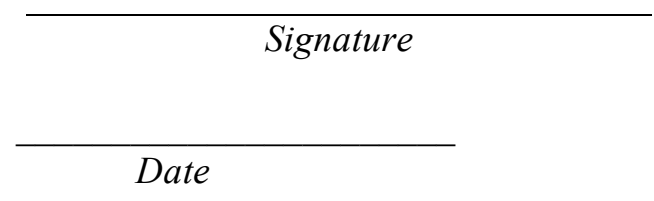

Date

A copy of this consent will be left with you, and a copy will be taken by the researcher. 


\section{Pre-Task Questionnaire}

Participant ID:

These statements are related to your experience in environmental data analysis and familiarity with similar software systems. Your answer to these questions will allow for accurate analysis of the data collected during the study.

1. How would you rate your experience with environmental data analysis?

$\square$ None $\quad \square$ Low $\quad \square$ Medium $\quad$ High

2. How would you rate your experience with the target domain (e.g., Small Scale Fisheries or Coastal Environments/Resiliency)?

$\square$ None $\quad \square$ Low $\quad \square$ Medium $\quad \square$ High

3. What are your preferred tools for analyzing data in your domain?

4. How would you rate your experience your preferred analysis tools?

$$
\text { aLow } \quad \square \text { Medium } \quad \text { High }
$$

5. How would you rate your experience with Geographic Information Systems (GIS)?

$\square$ None $\quad$ Low $\quad$ Medium $\quad$ High

6. How would you rate your experience with visualizations systems and methods?

$\square$ None $\quad \square$ Low $\quad \square$ Medium $\quad$ High

7. How would you rate your experience with analyzing environmental data using visualization systems?

aNone $\quad$ Low $\quad \square$ Medium $\quad$ High

8. How would you rate your experience with parallel coordinate plots?

$\square$ None $\quad$ Low $\quad \square$ Medium $\quad$ High 


\section{Post-Task Questionnaire}

\section{Parallel Coordinates - High Dimensional Visualization}

Participant ID:

These statements are related to your use of the parallel coordinates that visualize high dimensional data, along with the features to brush/filter the data across multiple dimensions, zoom in/out on multiple dimensions, reorder the dimensions, and highlight individual data points.

\begin{tabular}{|l|c|c|c|c|c|}
\hline General exploration of the data & $\begin{array}{c}\text { Strongly } \\
\text { Disagree }\end{array}$ & Disagree & Neutral & Agree & $\begin{array}{c}\text { Strongly } \\
\text { Agree }\end{array}$ \\
\hline $\begin{array}{l}\text { I was able to explore different subsets of the high } \\
\text { dimensional geotemporal data using the parallel } \\
\text { coordinates. }\end{array}$ & 1 & 2 & 3 & 4 & 5 \\
\hline $\begin{array}{l}\text { The parallel coordinates helped me to understand the high } \\
\text { dimensional geotemporal data. }\end{array}$ & 1 & 2 & 3 & 4 & 5 \\
\hline $\begin{array}{l}\text { The parallel coordinates helped me to explore the high } \\
\text { dimensional geotemporal data. }\end{array}$ & 1 & 2 & 3 & 4 & 5 \\
\hline $\begin{array}{l}\text { The parallel coordinates helped me to detect patterns and } \\
\text { trends in the high dimensional geotemporal data. }\end{array}$ & 1 & 2 & 3 & 4 & 5 \\
\hline $\begin{array}{l}\text { Using the parallel coordinates allowed me to find relevant } \\
\text { information in the high dimensional geotemporal data. }\end{array}$ & 1 & 2 & 3 & 4 & 5 \\
\hline
\end{tabular}

\begin{tabular}{|c|c|c|c|c|c|}
\hline Detailed analysis of the data & $\begin{array}{l}\text { Strongly } \\
\text { Disagree }\end{array}$ & Disagree & Neutral & Agree & $\begin{array}{l}\text { Strongly } \\
\text { Agree }\end{array}$ \\
\hline $\begin{array}{l}\text { I was able to understand relations between dimensions in } \\
\text { the data using the parallel coordinates. }\end{array}$ & 1 & 2 & 3 & 4 & 5 \\
\hline $\begin{array}{l}\text { I was able to understand the distribution of the data using } \\
\text { the parallel coordinates. }\end{array}$ & 1 & 2 & 3 & 4 & 5 \\
\hline $\begin{array}{l}\text { I was able to identify information that I already knew using } \\
\text { the parallel coordinates. }\end{array}$ & 1 & 2 & 3 & 4 & 5 \\
\hline $\begin{array}{l}\text { I was able to locate new information that I didn't previously } \\
\text { know using the parallel coordinates. }\end{array}$ & 1 & 2 & 3 & 4 & 5 \\
\hline $\begin{array}{l}\text { I was able to distinguish between different data points and } \\
\text { objects in the data set using the parallel coordinates. }\end{array}$ & 1 & 2 & 3 & 4 & 5 \\
\hline $\begin{array}{l}\text { I was able to compare different parameters (dimensions) } \\
\text { using the parallel coordinates. }\end{array}$ & 1 & 2 & 3 & 4 & 5 \\
\hline $\begin{array}{l}\text { I was able to compare different data points using the parallel } \\
\text { coordinates. }\end{array}$ & 1 & 2 & 3 & 4 & 5 \\
\hline $\begin{array}{l}\text { I was able to identify relations between multi-dimensional data } \\
\text { and their geo-locations using the parallel coordinates. }\end{array}$ & 1 & 2 & 3 & 4 & 5 \\
\hline $\begin{array}{l}\text { I was able to understand the correlations between different } \\
\text { parameters using the parallel coordinates. }\end{array}$ & 1 & 2 & 3 & 4 & 5 \\
\hline
\end{tabular}




\begin{tabular}{|l|c|c|c|c|c|}
\hline Job relevance & $\begin{array}{c}\text { Strongly } \\
\text { Disagree }\end{array}$ & Disagree & Neutral & Agree & $\begin{array}{c}\text { Strongly } \\
\text { Agree }\end{array}$ \\
\hline $\begin{array}{l}\text { I found the parallel coordinates relevant when analyzing } \\
\text { high dimensional geotemporal data. }\end{array}$ & 1 & 2 & 3 & 4 & 5 \\
\hline $\begin{array}{l}\text { I found the parallel coordinates important when analyzing } \\
\text { high dimensional geotemporal data. }\end{array}$ & 1 & 2 & 3 & 4 & 5 \\
\hline
\end{tabular}

\begin{tabular}{|c|c|c|c|c|c|}
\hline Perceived ease of use & $\begin{array}{l}\text { Strongly } \\
\text { Disagree }\end{array}$ & Disagree & Neutral & Agree & $\begin{array}{l}\text { Strongly } \\
\text { Agree }\end{array}$ \\
\hline $\begin{array}{l}\text { My interaction with the parallel coordinates is clear and } \\
\text { understandable. }\end{array}$ & 1 & 2 & 3 & 4 & 5 \\
\hline I find the parallel coordinates easy to use. & 1 & 2 & 3 & 4 & 5 \\
\hline $\begin{array}{l}\text { It was easy for me to become skilful at using the parallel } \\
\text { coordinates. }\end{array}$ & 1 & 2 & 3 & 4 & 5 \\
\hline $\begin{array}{l}\text { I found it easy to get the parallel coordinates to do what I } \\
\text { wanted it to do. }\end{array}$ & 1 & 2 & 3 & 4 & 5 \\
\hline $\begin{array}{l}\text { Learning to operate the parallel coordinates was easy for } \\
\text { me. }\end{array}$ & 1 & 2 & 3 & 4 & 5 \\
\hline $\begin{array}{l}\text { I found the parallel coordinates to be flexible to interact } \\
\text { with. }\end{array}$ & 1 & 2 & 3 & 4 & 5 \\
\hline
\end{tabular}

\begin{tabular}{|c|c|c|c|c|c|}
\hline Perceived usefulness & $\begin{array}{l}\text { Strongly } \\
\text { Disagree }\end{array}$ & Disagree & Neutral & Agree & $\begin{array}{l}\text { Strongly } \\
\text { Agree }\end{array}$ \\
\hline $\begin{array}{l}\text { Using the parallel coordinates improves my performance in } \\
\text { analyzing high dimensional geotemporal data. }\end{array}$ & 1 & 2 & 3 & 4 & 5 \\
\hline $\begin{array}{l}\text { Using the parallel coordinates to analyze high dimensional } \\
\text { geotemporal data increased my productivity. }\end{array}$ & 1 & 2 & 3 & 4 & 5 \\
\hline $\begin{array}{l}\text { Using the parallel coordinates enhances my effectiveness in } \\
\text { analyzing high dimensional geotemporal data. }\end{array}$ & 1 & 2 & 3 & 4 & 5 \\
\hline $\begin{array}{l}\text { I find the parallel coordinates useful in analyzing high } \\
\text { dimensional geotemporal data. }\end{array}$ & 1 & 2 & 3 & 4 & 5 \\
\hline $\begin{array}{l}\text { Using the parallel coordinates made it easier for me to analyze } \\
\text { high dimensional geotemporal data. }\end{array}$ & 1 & 2 & 3 & 4 & 5 \\
\hline $\begin{array}{l}\text { Using the parallel coordinates in my analysis of high } \\
\text { dimensional geotemporal data enabled me to accomplish my } \\
\text { tasks more quickly. }\end{array}$ & 1 & 2 & 3 & 4 & 5 \\
\hline
\end{tabular}

\begin{tabular}{|l|c|c|c|c|c|}
\hline Output quality & $\begin{array}{c}\text { Strongly } \\
\text { Disagree }\end{array}$ & Disagree & Neutral & Agree & $\begin{array}{c}\text { Strongly } \\
\text { Agree }\end{array}$ \\
\hline $\begin{array}{l}\text { The quality of the output I get from the parallel coordinates is } \\
\text { high. }\end{array}$ & 1 & 2 & 3 & 4 & 5 \\
\hline $\begin{array}{l}\text { I have no problem with the quality of the parallel coordinates } \\
\text { output. }\end{array}$ & 1 & 2 & 3 & 4 & 5 \\
\hline
\end{tabular}




\begin{tabular}{|c|c|c|c|c|c|}
\hline $\begin{array}{l}\text { I have no difficulty telling others about the result of using the } \\
\text { parallel coordinates. }\end{array}$ & 1 & 2 & 3 & 4 & 5 \\
\hline $\begin{array}{l}\text { I believe I could communicate to others the consequences of } \\
\text { using the parallel coordinates. }\end{array}$ & 1 & 2 & 3 & 4 & 5 \\
\hline $\begin{array}{l}\text { The results of using the parallel coordinates are apparent to } \\
\text { me. }\end{array}$ & 1 & 2 & 3 & 4 & 5 \\
\hline $\begin{array}{l}\text { I would have difficulty explaining why using the parallel } \\
\text { coordinates may or may not be beneficial. }\end{array}$ & 1 & 2 & 3 & 4 & 5 \\
\hline
\end{tabular}




\section{Geo-visualisation (Thematic Map/ Hex-binning)}

These statements are related to your use of the geo-visualization, which visualizes the geospatial distribution of the data both as a thematic map and using hex-binning. This includes the features to zoom, pan, and filter data.

\begin{tabular}{|l|c|c|c|c|c|}
\hline General exploration of the data & $\begin{array}{c}\text { Strongly } \\
\text { Disagree }\end{array}$ & Disagree & Neutral & $\begin{array}{c}\text { Agree } \\
\text { Strongly } \\
\text { Agree }\end{array}$ \\
\hline $\begin{array}{l}\text { I was able to explore different subsets of the high } \\
\text { dimensional geotemporal data using the geo- } \\
\text { visualization. }\end{array}$ & 1 & 2 & 3 & 4 & 5 \\
\hline $\begin{array}{l}\text { The geo-visualization helped me to understand the high } \\
\text { dimensional geotemporal data. }\end{array}$ & 1 & 2 & 3 & 4 & 5 \\
\hline $\begin{array}{l}\text { The geo-visualization helped me to explore the high } \\
\text { dimensional geotemporal data. }\end{array}$ & 1 & 2 & 3 & 4 & 5 \\
\hline $\begin{array}{l}\text { The geo-visualization helped me to detect patterns and } \\
\text { trends in the high dimensional geotemporal data. }\end{array}$ & 1 & 2 & 3 & 4 & 5 \\
\hline $\begin{array}{l}\text { Using the geo-visualization allowed me to find relevant } \\
\text { information in the high dimensional geotemporal data. }\end{array}$ & 1 & 2 & 3 & 4 & 5 \\
\hline
\end{tabular}

\begin{tabular}{|c|c|c|c|c|c|}
\hline Detailed analysis of data & $\begin{array}{l}\text { Strongly } \\
\text { Disagree }\end{array}$ & Disagree & Neutral & Agree & $\begin{array}{l}\text { Strongly } \\
\text { Agree }\end{array}$ \\
\hline $\begin{array}{l}\text { I was able to understand relations between dimensions in } \\
\text { the data using the geo-visualization. }\end{array}$ & 1 & 2 & 3 & 4 & 5 \\
\hline $\begin{array}{l}\text { I was able to understand the distribution of the data using } \\
\text { the geo-visualization. }\end{array}$ & 1 & 2 & 3 & 4 & 5 \\
\hline $\begin{array}{l}\text { I was able to identify information that I already knew } \\
\text { using the geo-visualization. }\end{array}$ & 1 & 2 & 3 & 4 & 5 \\
\hline $\begin{array}{l}\text { I was able to locate new information that I didn't } \\
\text { previously know using the geo-visualization. }\end{array}$ & 1 & 2 & 3 & 4 & 5 \\
\hline $\begin{array}{l}\text { I was able to distinguish between different data points } \\
\text { and objects in the data set using the geo-visualization. }\end{array}$ & 1 & 2 & 3 & 4 & 5 \\
\hline $\begin{array}{l}\text { I was able to compare different parameters (dimensions) } \\
\text { using the geo-visualization. }\end{array}$ & 1 & 2 & 3 & 4 & 5 \\
\hline $\begin{array}{l}\text { I was able to compare different data points using the geo- } \\
\text { visualization. }\end{array}$ & 1 & 2 & 3 & 4 & 5 \\
\hline $\begin{array}{l}\text { I was able to identify relations between multi-dimensional } \\
\text { data and their geo-locations using the geo-visualization. }\end{array}$ & 1 & 2 & 3 & 4 & 5 \\
\hline $\begin{array}{l}\text { I was able to understand the correlations between different } \\
\text { parameters using the geo-visualization. }\end{array}$ & 1 & 2 & 3 & 4 & 5 \\
\hline
\end{tabular}

\begin{tabular}{|c|c|c|c|c|c|}
\hline Job relevance & $\begin{array}{l}\text { Strongly } \\
\text { Disagree }\end{array}$ & Disagree & Neutral & Agree & $\begin{array}{c}\text { Strongly } \\
\text { Agree }\end{array}$ \\
\hline $\begin{array}{l}\text { I found the geo-visualization relevant when analyzing high } \\
\text { dimensional geotemporal data. }\end{array}$ & 1 & 2 & 3 & 4 & 5 \\
\hline $\begin{array}{l}\text { I found the geo-visualization important when analyzing } \\
\text { high dimensional geotemporal data. }\end{array}$ & 1 & 2 & 3 & 4 & 5 \\
\hline
\end{tabular}




\begin{tabular}{|c|c|c|c|c|c|}
\hline Perceived ease of use & $\begin{array}{l}\text { Strongly } \\
\text { Disagree }\end{array}$ & Disagree & Neutral & Agree & $\begin{array}{c}\text { Strongly } \\
\text { Agree }\end{array}$ \\
\hline $\begin{array}{l}\text { My interaction with the geo-visualization is clear and } \\
\text { understandable. }\end{array}$ & 1 & 2 & 3 & 4 & 5 \\
\hline I find the geo-visualization easy to use. & 1 & 2 & 3 & 4 & 5 \\
\hline $\begin{array}{l}\text { It was easy for me to become skilful at using the geo- } \\
\text { visualization. }\end{array}$ & 1 & 2 & 3 & 4 & 5 \\
\hline $\begin{array}{l}\text { I found it easy to get the geo-visualization to do what I } \\
\text { wanted it to do. }\end{array}$ & 1 & 2 & 3 & 4 & 5 \\
\hline $\begin{array}{l}\text { Learning to operate the geo-visualization was easy for } \\
\text { me. }\end{array}$ & 1 & 2 & 3 & 4 & 5 \\
\hline $\begin{array}{l}\text { I found the geo-visualization to be flexible to interact } \\
\text { with. }\end{array}$ & 1 & 2 & 3 & 4 & 5 \\
\hline
\end{tabular}

\begin{tabular}{|c|c|c|c|c|c|}
\hline Perceived usefulness & $\begin{array}{l}\text { Strongly } \\
\text { Disagree }\end{array}$ & Disagree & Neutral & Agree & $\begin{array}{l}\text { Strongly } \\
\text { Agree }\end{array}$ \\
\hline $\begin{array}{l}\text { Using the geo-visualization improves my performance in } \\
\text { analyzing high dimensional geotemporal data. }\end{array}$ & 1 & 2 & 3 & 4 & 5 \\
\hline $\begin{array}{l}\text { Using the geo-visualization to analyze high dimensional } \\
\text { geotemporal data increased my productivity. }\end{array}$ & 1 & 2 & 3 & 4 & 5 \\
\hline $\begin{array}{l}\text { Using the geo-visualization enhances my effectiveness in } \\
\text { analyzing high dimensional geotemporal data. }\end{array}$ & 1 & 2 & 3 & 4 & 5 \\
\hline $\begin{array}{l}\text { I find the geo-visualization useful in analyzing high } \\
\text { dimensional geotemporal data. }\end{array}$ & 1 & 2 & 3 & 4 & 5 \\
\hline $\begin{array}{l}\text { Using the geo-visualization made it easier for me to } \\
\text { analyze high dimensional geotemporal data. }\end{array}$ & 1 & 2 & 3 & 4 & 5 \\
\hline $\begin{array}{l}\text { Using the geo-visualization in my analysis of high } \\
\text { dimensional geotemporal data enabled me to } \\
\text { accomplish my tasks more quickly. }\end{array}$ & 1 & 2 & 3 & 4 & 5 \\
\hline
\end{tabular}

\begin{tabular}{|c|c|c|c|c|c|}
\hline Output quality & $\begin{array}{l}\text { Strongly } \\
\text { Disagree }\end{array}$ & Disagree & Neutral & Agree & $\begin{array}{l}\text { Strongly } \\
\text { Agree }\end{array}$ \\
\hline $\begin{array}{l}\text { The quality of the output I get from the geo-visualization } \\
\text { is high. }\end{array}$ & 1 & 2 & 3 & 4 & 5 \\
\hline $\begin{array}{l}\text { I have no problem with the quality of the geo-visualization } \\
\text { output. }\end{array}$ & 1 & 2 & 3 & 4 & 5 \\
\hline $\begin{array}{l}\text { I have no difficulty telling other about the result of using } \\
\text { the geo-visualization. }\end{array}$ & 1 & 2 & 3 & 4 & 5 \\
\hline $\begin{array}{l}\text { I believe I could communicate to others the } \\
\text { consequences of using the geo-visualization. }\end{array}$ & 1 & 2 & 3 & 4 & 5 \\
\hline $\begin{array}{l}\text { The results of using the geo-visualization are apparent to } \\
\text { me. }\end{array}$ & 1 & 2 & 3 & 4 & 5 \\
\hline $\begin{array}{l}\text { I would have difficulty explaining why using the geo- } \\
\text { visualization may or may not be beneficial. }\end{array}$ & 1 & 2 & 3 & 4 & 5 \\
\hline
\end{tabular}




\section{Visualization of the Micro-Statistical Information}

These statements are related to your use of the visualization of the micro statistical information (on top of the parallel coordinates) along with the features to re-compute the statistics with each change in the parallel coordinate filtering.

\begin{tabular}{|c|c|c|c|c|c|}
\hline General exploration of the data & $\begin{array}{l}\text { Strongly } \\
\text { Disagree }\end{array}$ & Disagree & Neutral & Agree & $\begin{array}{c}\text { Strongly } \\
\text { Agree }\end{array}$ \\
\hline $\begin{array}{l}\text { I was able to explore different subsets of the high } \\
\text { dimensional geotemporal data using the visualization of } \\
\text { the micro-statistical information. }\end{array}$ & 1 & 2 & 3 & 4 & 5 \\
\hline $\begin{array}{l}\text { The visualization of the micro-statistical information } \\
\text { helped me to understand the high dimensional } \\
\text { geotemporal data. }\end{array}$ & 1 & 2 & 3 & 4 & 5 \\
\hline $\begin{array}{l}\text { The visualization of the micro-statistical information } \\
\text { helped me to explore the high dimensional geotemporal } \\
\text { data. }\end{array}$ & 1 & 2 & 3 & 4 & 5 \\
\hline $\begin{array}{l}\text { The visualization of the micro-statistical information } \\
\text { helped me to detect patterns and trends in the high } \\
\text { dimensional geotemporal data. }\end{array}$ & 1 & 2 & 3 & 4 & 5 \\
\hline $\begin{array}{l}\text { Using the visualization of the micro-statistical information } \\
\text { allowed me to find relevant information in the high } \\
\text { dimensional geotemporal data. }\end{array}$ & 1 & 2 & 3 & 4 & 5 \\
\hline
\end{tabular}

\begin{tabular}{|c|c|c|c|c|c|}
\hline Detailed analysis of data & $\begin{array}{l}\text { Strongly } \\
\text { Disagree }\end{array}$ & Disagree & Neutral & Agree & $\begin{array}{l}\text { Strongly } \\
\text { Agree }\end{array}$ \\
\hline $\begin{array}{l}\text { I was able to understand relations between dimensions in } \\
\text { the data using the visualization of the micro-statistical } \\
\text { information. }\end{array}$ & 1 & 2 & 3 & 4 & 5 \\
\hline $\begin{array}{l}\text { I was able to understand the distribution of the data using } \\
\text { the visualization of the micro-statistical information. }\end{array}$ & 1 & 2 & 3 & 4 & 5 \\
\hline $\begin{array}{l}\text { I was able to identify information that I already knew using } \\
\text { the visualization of the micro-statistical information. }\end{array}$ & 1 & 2 & 3 & 4 & 5 \\
\hline $\begin{array}{l}\text { I was able to locate new information that I didn't previously } \\
\text { know using the visualization of the micro-statistical } \\
\text { information. }\end{array}$ & 1 & 2 & 3 & 4 & 5 \\
\hline $\begin{array}{l}\text { I was able to distinguish between different data points and } \\
\text { objects in the data set using the visualization of the micro- } \\
\text { statistical information. }\end{array}$ & 1 & 2 & 3 & 4 & 5 \\
\hline $\begin{array}{l}\text { I was able to compare different parameters (dimensions) } \\
\text { using the visualization of the micro-statistical information. }\end{array}$ & 1 & 2 & 3 & 4 & 5 \\
\hline $\begin{array}{l}\text { I was able to compare different data points using the } \\
\text { visualization of the micro-statistical information. }\end{array}$ & 1 & 2 & 3 & 4 & 5 \\
\hline $\begin{array}{l}\text { I was able to identify relations between multi-dimensional } \\
\text { data and their geo-locations using the visualization of the } \\
\text { micro-statistical information. }\end{array}$ & 1 & 2 & 3 & 4 & 5 \\
\hline
\end{tabular}




\begin{tabular}{|l|l|l|l|l|}
\hline $\begin{array}{l}\text { I was able to understand the correlations between different } \\
\text { parameters using the visualization of the micro-statistical } \\
\text { information. }\end{array}$ & 1 & 2 & 3 & 4 \\
\hline
\end{tabular}

\begin{tabular}{|c|c|c|c|c|c|}
\hline Job relevance & $\begin{array}{l}\text { Strongly } \\
\text { Disagree }\end{array}$ & Disagree & Neutral & Agree & $\begin{array}{c}\text { Strongly } \\
\text { Agree }\end{array}$ \\
\hline $\begin{array}{l}\text { I found the visualization of the micro-statistical } \\
\text { information relevant when analyzing high dimensional } \\
\text { geotemporal data. }\end{array}$ & 1 & 2 & 3 & 4 & 5 \\
\hline $\begin{array}{l}\text { I found the visualization of the micro-statistical } \\
\text { information important when analyzing high dimensional } \\
\text { geotemporal data. }\end{array}$ & 1 & 2 & 3 & 4 & 5 \\
\hline
\end{tabular}

\begin{tabular}{|c|c|c|c|c|c|}
\hline Perceived ease of use & $\begin{array}{l}\text { Strongly } \\
\text { Disagree }\end{array}$ & Disagree & Neutral & Agree & $\begin{array}{l}\text { Strongly } \\
\text { Agree }\end{array}$ \\
\hline $\begin{array}{l}\text { My interaction with the visualization of the micro- } \\
\text { statistical information is clear and understandable. }\end{array}$ & 1 & 2 & 3 & 4 & 5 \\
\hline $\begin{array}{l}\text { I find the visualization of the micro-statistical information } \\
\text { easy to use. }\end{array}$ & 1 & 2 & 3 & 4 & 5 \\
\hline $\begin{array}{l}\text { I found it easy to get the visualization of the micro- } \\
\text { statistical information to do what I wanted it to do. }\end{array}$ & 1 & 2 & 3 & 4 & 5 \\
\hline $\begin{array}{l}\text { Learning to operate the visualization of the micro- } \\
\text { statistical information was easy for me. }\end{array}$ & 1 & 2 & 3 & 4 & 5 \\
\hline
\end{tabular}




\begin{tabular}{|c|c|c|c|c|c|}
\hline Perceived usefulness & $\begin{array}{l}\text { Strongly } \\
\text { Disagree }\end{array}$ & Disagree & Neutral & Agree & $\begin{array}{c}\text { Strongly } \\
\text { Agree }\end{array}$ \\
\hline $\begin{array}{l}\text { Using the visualization of the micro-statistical information } \\
\text { improves my performance in analyzing high dimensional } \\
\text { geotemporal data. }\end{array}$ & 1 & 2 & 3 & 4 & 5 \\
\hline $\begin{array}{l}\text { Using the visualization of the micro-statistical information } \\
\text { to analyze high dimensional geotemporal data increased } \\
\text { my productivity. }\end{array}$ & 1 & 2 & 3 & 4 & 5 \\
\hline $\begin{array}{l}\text { Using the visualization of the micro-statistical information } \\
\text { enhances my effectiveness in analyzing high dimensional } \\
\text { geotemporal data. }\end{array}$ & 1 & 2 & 3 & 4 & 5 \\
\hline $\begin{array}{l}\text { I find the visualization of the micro-statistical information } \\
\text { useful in analyzing high dimensional geotemporal data. }\end{array}$ & 1 & 2 & 3 & 4 & 5 \\
\hline $\begin{array}{l}\text { Using the visualization of the micro-statistical information } \\
\text { made it easier for me to analyze high dimensional } \\
\text { geotemporal data. }\end{array}$ & 1 & 2 & 3 & 4 & 5 \\
\hline $\begin{array}{l}\text { Using the visualization of the micro-statistical information } \\
\text { in my analysis of high dimensional geotemporal data } \\
\text { enabled me to accomplish my tasks more quickly. }\end{array}$ & 1 & 2 & 3 & 4 & 5 \\
\hline
\end{tabular}

\begin{tabular}{|c|c|c|c|c|c|}
\hline Output quality & $\begin{array}{l}\text { Strongly } \\
\text { Disagree }\end{array}$ & Disagree & Neutral & Agree & $\begin{array}{c}\text { Strongly } \\
\text { Agree }\end{array}$ \\
\hline $\begin{array}{l}\text { The quality of the output I get from the visualization of the } \\
\text { micro-statistical information is high. }\end{array}$ & 1 & 2 & 3 & 4 & 5 \\
\hline $\begin{array}{l}\text { I have no problem with the quality of the visualization of } \\
\text { the micro-statistical information output. }\end{array}$ & 1 & 2 & 3 & 4 & 5 \\
\hline $\begin{array}{l}\text { I have no difficulty telling other about the result of using the } \\
\text { visualization of the micro-statistical information. }\end{array}$ & 1 & 2 & 3 & 4 & 5 \\
\hline $\begin{array}{l}\text { I believe I could communicate to others the consequences } \\
\text { of using the visualization of the micro-statistical } \\
\text { information. }\end{array}$ & 1 & 2 & 3 & 4 & 5 \\
\hline $\begin{array}{l}\text { The results of using the visualization of the micro- } \\
\text { statistical information are apparent to me. }\end{array}$ & 1 & 2 & 3 & 4 & 5 \\
\hline $\begin{array}{l}\text { I would have difficulty explaining why using the } \\
\text { visualization of the micro-statistical information may or } \\
\text { may not be beneficial. }\end{array}$ & 1 & 2 & 3 & 4 & 5 \\
\hline
\end{tabular}




\section{Interactive Analysis Features (dynamic investigation/dynamic encoding)}

These statements are related to your use of the interactive analysis features in the system including the scatter plot and changing the visual encoding (colour and size) in the different views.

\begin{tabular}{|l|c|c|c|c|c|}
\hline General exploration of the data & $\begin{array}{c}\text { Strongly } \\
\text { Disagree }\end{array}$ & Disagree & Neutral & $\begin{array}{c}\text { Agree } \\
\text { Strongly } \\
\text { Agree }\end{array}$ \\
\hline $\begin{array}{l}\text { I was able to explore different subsets of the high } \\
\text { dimensional geotemporal data using the interactive } \\
\text { analysis features. }\end{array}$ & 1 & 2 & 3 & 4 & 5 \\
\hline $\begin{array}{l}\text { The interactive analysis features helped me to understand } \\
\text { the high dimensional geotemporal data. }\end{array}$ & 1 & 2 & 3 & 4 & 5 \\
\hline $\begin{array}{l}\text { The interactive analysis features helped me to explore the } \\
\text { high dimensional geotemporal data. }\end{array}$ & 1 & 2 & 3 & 4 & 5 \\
\hline $\begin{array}{l}\text { The interactive analysis features helped me to detect } \\
\text { patterns and trends in the high dimensional geotemporal } \\
\text { data. }\end{array}$ & 1 & 2 & 3 & 4 & 5 \\
\hline $\begin{array}{l}\text { Using the interactive analysis features allowed me to find } \\
\text { relevant information in the high dimensional geotemporal } \\
\text { data. }\end{array}$ & 1 & 2 & 3 & 4 & 5 \\
\hline
\end{tabular}

\begin{tabular}{|l|c|c|c|c|c|}
\hline Detailed analysis of data & $\begin{array}{c}\text { Strongly } \\
\text { Disagree }\end{array}$ & Disagree & Neutral & Agree & $\begin{array}{c}\text { Strongly } \\
\text { Agree }\end{array}$ \\
\hline $\begin{array}{l}\text { I was able to understand relations between dimensions in } \\
\text { the data using the interactive analysis features. }\end{array}$ & 1 & 2 & 3 & 4 & 5 \\
\hline $\begin{array}{l}\text { I was able to understand the distribution of the data using } \\
\text { the interactive analysis features. }\end{array}$ & 1 & 2 & 3 & 4 & 5 \\
\hline $\begin{array}{l}\text { I was able to identify information that I already knew using } \\
\text { the interactive analysis features. }\end{array}$ & 1 & 2 & 3 & 4 & 5 \\
\hline $\begin{array}{l}\text { I was able to locate new information that I didn't } \\
\text { previously know using the interactive analysis features. }\end{array}$ & 1 & 2 & 3 & 4 & 5 \\
\hline $\begin{array}{l}\text { I was able to distinguish between different data points and } \\
\text { objects in the data set using the interactive analysis } \\
\text { features. }\end{array}$ & 1 & 2 & 3 & 4 & 5 \\
\hline $\begin{array}{l}\text { I was able to compare different parameters (dimensions) } \\
\text { using the interactive analysis features. }\end{array}$ & 1 & 2 & 3 & 4 & 5 \\
\hline $\begin{array}{l}\text { I was able to compare different data points using the } \\
\text { interactive analysis features. }\end{array}$ & 1 & 2 & 3 & 4 & 5 \\
\hline $\begin{array}{l}\text { I was able to identify relations between multi-dimensional } \\
\text { data and their geo-locations using the interactive analysis } \\
\text { features. }\end{array}$ & 1 & 2 & 3 & 4 & 5 \\
\hline $\begin{array}{l}\text { I was able to understand the correlations between different } \\
\text { parameters using the interactive analysis features. }\end{array}$ & 1 & 2 & 3 & 4 & 5 \\
\hline
\end{tabular}




\begin{tabular}{|c|c|c|c|c|c|}
\hline Job relevance & $\begin{array}{l}\text { Strongly } \\
\text { Disagree }\end{array}$ & Disagree & Neutral & Agree & $\begin{array}{l}\text { Strongly } \\
\text { Agree }\end{array}$ \\
\hline $\begin{array}{l}\text { I found interactive analysis features relevant when } \\
\text { analyzing high dimensional geotemporal data. }\end{array}$ & 1 & 2 & 3 & 4 & 5 \\
\hline $\begin{array}{l}\text { I found interactive analysis features important when } \\
\text { analyzing high dimensional geotemporal data. }\end{array}$ & 1 & 2 & 3 & 4 & 5 \\
\hline
\end{tabular}

\begin{tabular}{|c|c|c|c|c|c|}
\hline Perceived ease of use & $\begin{array}{l}\text { Strongly } \\
\text { Disagree }\end{array}$ & Disagree & Neutral & Agree & $\begin{array}{l}\text { Strongly } \\
\text { Agree }\end{array}$ \\
\hline $\begin{array}{l}\text { My interaction with the interactive analysis features is } \\
\text { clear and understandable. }\end{array}$ & 1 & 2 & 3 & 4 & 5 \\
\hline I find the interactive analysis features easy to use. & 1 & 2 & 3 & 4 & 5 \\
\hline $\begin{array}{l}\text { It was easy for me to become skilful at using the } \\
\text { interactive analysis features. }\end{array}$ & 1 & 2 & 3 & 4 & 5 \\
\hline $\begin{array}{l}\text { I found it easy to get the interactive analysis features to } \\
\text { do what I wanted it to do. }\end{array}$ & 1 & 2 & 3 & 4 & 5 \\
\hline $\begin{array}{l}\text { Learning to operate the interactive analysis features was } \\
\text { easy for me. }\end{array}$ & 1 & 2 & 3 & 4 & 5 \\
\hline $\begin{array}{l}\text { I found the interactive analysis features to be flexible to } \\
\text { interact with. }\end{array}$ & 1 & 2 & 3 & 4 & 5 \\
\hline
\end{tabular}

\begin{tabular}{|l|c|c|c|}
\hline Perceived usefulness & $\begin{array}{c}\text { Strongly } \\
\text { Disagree }\end{array}$ & $\begin{array}{c}\text { Disagree } \\
\text { Neutral }\end{array}$ & $\begin{array}{c}\text { Agree } \\
\text { Agree }\end{array}$ \\
\hline $\begin{array}{l}\text { Using the interactive analysis features improve my } \\
\text { performance in analyzing high dimensional geotemporal } \\
\text { data. }\end{array}$ & 1 & 2 & 3 \\
\hline $\begin{array}{l}\text { Using the interactive analysis features to analyze high } \\
\text { dimensional geotemporal data increased my productivity. }\end{array}$ & 1 & 2 & 3 \\
\hline $\begin{array}{l}\text { Using the interactive analysis features enhance my } \\
\text { effectiveness in analyzing high dimensional geotemporal } \\
\text { data. }\end{array}$ & 1 & 2 & 3 \\
\hline $\begin{array}{l}\text { I find the interactive analysis features useful in analyzing } \\
\text { high dimensional geotemporal data. }\end{array}$ & 1 & 2 & 5 \\
\hline $\begin{array}{l}\text { Using the interactive analysis features made it easier for } \\
\text { me to analyze high dimensional geotemporal data. }\end{array}$ & 1 & 2 & 3 \\
\hline $\begin{array}{l}\text { Using the interactive analysis features in my analysis of } \\
\text { high dimensional geotemporal data enabled me to } \\
\text { accomplish my tasks more quickly. }\end{array}$ & 1 & 2 & 3 \\
\hline
\end{tabular}

\begin{tabular}{|l|c|c|c|c|}
\hline Output quality & $\begin{array}{c}\text { Strongly } \\
\text { Disagree }\end{array}$ & $\begin{array}{c}\text { Disagree } \\
\text { Neutral }\end{array}$ & $\begin{array}{c}\text { Agree } \\
\text { Agree }\end{array}$ \\
\hline $\begin{array}{l}\text { The quality of the output I get from the interactive analysis } \\
\text { features is high. }\end{array}$ & 1 & 2 & 3 & 4 \\
\hline I have no problem with the quality of the interactive & 1 & 2 & 3 & 4 \\
\hline
\end{tabular}




\begin{tabular}{|c|c|c|c|c|c|}
\hline analysis features output. & & & & & \\
\hline $\begin{array}{l}\text { I have no difficulty telling other about the result of using the } \\
\text { interactive analysis features. }\end{array}$ & 1 & 2 & 3 & 4 & 5 \\
\hline $\begin{array}{l}\text { I believe I could communicate to others the consequences } \\
\text { of using the interactive analysis features. }\end{array}$ & 1 & 2 & 3 & 4 & 5 \\
\hline $\begin{array}{l}\text { The results of using the interactive analysis features are } \\
\text { apparent to me. }\end{array}$ & 1 & 2 & 3 & 4 & 5 \\
\hline $\begin{array}{l}\text { I would have difficulty explaining why using the interactive } \\
\text { analysis features may or may not be beneficial. }\end{array}$ & 1 & 2 & 3 & 4 & 5 \\
\hline
\end{tabular}




\section{Outlier Detection}

These statements are related to your use of the outlier detection algorithm along with the features to explore outliers or remove them from the analysis.

\begin{tabular}{|l|c|c|c|c|c|}
\hline General exploration of the data & $\begin{array}{c}\text { Strongly } \\
\text { Disagree }\end{array}$ & Disagree & Neutral & $\begin{array}{c}\text { Agree } \\
\text { Strongly } \\
\text { Agree }\end{array}$ \\
\hline $\begin{array}{l}\text { I was able to explore different subsets of the high } \\
\text { dimensional geotemporal data using the outlier detection. }\end{array}$ & 1 & 2 & 3 & 4 & 5 \\
\hline $\begin{array}{l}\text { The outlier detection helped me to understand the high } \\
\text { dimensional geotemporal data. }\end{array}$ & 1 & 2 & 3 & 4 & 5 \\
\hline $\begin{array}{l}\text { The Outlier detection helped me to explore the high } \\
\text { dimensional geotemporal data. }\end{array}$ & 1 & 2 & 3 & 4 & 5 \\
\hline $\begin{array}{l}\text { The Outlier detection helped me to detect patterns and } \\
\text { trends in the high dimensional geotemporal data. }\end{array}$ & 1 & 2 & 3 & 4 & 5 \\
\hline $\begin{array}{l}\text { Using the outlier detection allowed me to find relevant } \\
\text { information in the high dimensional geotemporal data. }\end{array}$ & 1 & 2 & 3 & 4 & 5 \\
\hline
\end{tabular}

\begin{tabular}{|c|c|c|c|c|c|}
\hline Detailed analysis of data & $\begin{array}{l}\text { Strongly } \\
\text { Disagree }\end{array}$ & Disagree & Neutral & Agree & $\begin{array}{l}\text { Strongly } \\
\text { Agree }\end{array}$ \\
\hline $\begin{array}{l}\text { I was able to understand relations between dimensions in } \\
\text { the data using the outlier detection. }\end{array}$ & 1 & 2 & 3 & 4 & 5 \\
\hline $\begin{array}{l}\text { I was able to understand the distribution of the data using } \\
\text { the outlier detection. }\end{array}$ & 1 & 2 & 3 & 4 & 5 \\
\hline $\begin{array}{l}\text { I was able to identify information that I already knew using } \\
\text { the outlier detection. }\end{array}$ & 1 & 2 & 3 & 4 & 5 \\
\hline $\begin{array}{l}\text { I was able to locate new information that I didn't previously } \\
\text { know using the outlier detection. }\end{array}$ & 1 & 2 & 3 & 4 & 5 \\
\hline $\begin{array}{l}\text { I was able to distinguish between different data points and } \\
\text { objects in the data set using the outlier detection. }\end{array}$ & 1 & 2 & 3 & 4 & 5 \\
\hline $\begin{array}{l}\text { I was able to compare different parameters (dimensions) } \\
\text { using the outlier detection. }\end{array}$ & 1 & 2 & 3 & 4 & 5 \\
\hline $\begin{array}{l}\text { I was able to compare different data points using the outlier } \\
\text { detection. }\end{array}$ & 1 & 2 & 3 & 4 & 5 \\
\hline $\begin{array}{l}\text { I was able to identify relations between multi-dimensional } \\
\text { data and their geo-locations using the outlier detection. }\end{array}$ & 1 & 2 & 3 & 4 & 5 \\
\hline $\begin{array}{l}\text { I was able to understand the correlations between different } \\
\text { parameters using the outlier detection. }\end{array}$ & 1 & 2 & 3 & 4 & 5 \\
\hline
\end{tabular}

\begin{tabular}{|l|c|c|c|c|c|}
\hline Job relevance & $\begin{array}{c}\text { Strongly } \\
\text { Disagree }\end{array}$ & Disagree & Neutral & $\begin{array}{c}\text { Agree } \\
\text { Strongly } \\
\text { Agree }\end{array}$ \\
\hline $\begin{array}{l}\text { I found the outlier detection relevant when analyzing high } \\
\text { dimensional geotemporal data. }\end{array}$ & 1 & 2 & 3 & 4 & 5 \\
\hline $\begin{array}{l}\text { I found the outlier detection important when analyzing high } \\
\text { dimensional geotemporal data. }\end{array}$ & 1 & 2 & 3 & 4 & 5 \\
\hline
\end{tabular}




\begin{tabular}{|c|c|c|c|c|c|}
\hline Perceived ease of use & $\begin{array}{l}\text { Strongly } \\
\text { Disagree }\end{array}$ & Disagree & Neutral & Agree & $\begin{array}{c}\text { Strongly } \\
\text { Agree }\end{array}$ \\
\hline $\begin{array}{l}\text { My interaction with the outlier detection is clear and } \\
\text { understandable. }\end{array}$ & 1 & 2 & 3 & 4 & 5 \\
\hline I find the outlier detection easy to use. & 1 & 2 & 3 & 4 & 5 \\
\hline $\begin{array}{l}\text { It was easy for me to become skilful at using the outlier } \\
\text { detection. }\end{array}$ & 1 & 2 & 3 & 4 & 5 \\
\hline $\begin{array}{l}\text { I found it easy to get the outlier detection to do what I } \\
\text { wanted it to do. }\end{array}$ & 1 & 2 & 3 & 4 & 5 \\
\hline Learning to operate the outlier detection was easy for me. & 1 & 2 & 3 & 4 & 5 \\
\hline I found the outlier detection to be flexible to interact with. & 1 & 2 & 3 & 4 & 5 \\
\hline
\end{tabular}

\begin{tabular}{|c|c|c|c|c|c|}
\hline Perceived usefulness & $\begin{array}{l}\text { Strongly } \\
\text { Disagree }\end{array}$ & Disagree & Neutral & Agree & $\begin{array}{l}\text { Strongly } \\
\text { Agree }\end{array}$ \\
\hline $\begin{array}{l}\text { Using the outlier detection improves my performance in } \\
\text { analyzing high dimensional geotemporal data. }\end{array}$ & 1 & 2 & 3 & 4 & 5 \\
\hline $\begin{array}{l}\text { Using the outlier detection to analyze high dimensional } \\
\text { geotemporal data increased my productivity. }\end{array}$ & 1 & 2 & 3 & 4 & 5 \\
\hline $\begin{array}{l}\text { Using the outlier detection enhances my effectiveness in } \\
\text { analyzing high dimensional geotemporal data. }\end{array}$ & 1 & 2 & 3 & 4 & 5 \\
\hline $\begin{array}{l}\text { I find the outlier detection useful in analyzing high } \\
\text { dimensional geotemporal data. }\end{array}$ & 1 & 2 & 3 & 4 & 5 \\
\hline $\begin{array}{l}\text { Using the outlier detection made it easier for me to analyze } \\
\text { high dimensional geotemporal data. }\end{array}$ & 1 & 2 & 3 & 4 & 5 \\
\hline $\begin{array}{l}\text { Using the outlier detection in my analysis of high } \\
\text { dimensional geotemporal data enabled me to accomplish my } \\
\text { tasks more quickly. }\end{array}$ & 1 & 2 & 3 & 4 & 5 \\
\hline
\end{tabular}

\begin{tabular}{|c|c|c|c|c|c|}
\hline Output quality & $\begin{array}{l}\text { Strongly } \\
\text { Disagree }\end{array}$ & Disagree & Neutral & Agree & $\begin{array}{c}\text { Strongly } \\
\text { Agree }\end{array}$ \\
\hline $\begin{array}{l}\text { The quality of the output I get from the outlier detection is } \\
\text { high. }\end{array}$ & 1 & 2 & 3 & 4 & 5 \\
\hline $\begin{array}{l}\text { I have no problem with the quality of the outlier detection } \\
\text { output. }\end{array}$ & 1 & 2 & 3 & 4 & 5 \\
\hline $\begin{array}{l}\text { I have no difficulty telling other about the result of using the } \\
\text { outlier detection. }\end{array}$ & 1 & 2 & 3 & 4 & 5 \\
\hline $\begin{array}{l}\text { I believe I could communicate to others the consequences of } \\
\text { using the outlier detection. }\end{array}$ & 1 & 2 & 3 & 4 & 5 \\
\hline The results of using the outlier detection are apparent to me. & 1 & 2 & 3 & 4 & 5 \\
\hline $\begin{array}{l}\text { I would have difficulty explaining why using the outlier } \\
\text { detection may or may not be beneficial. }\end{array}$ & 1 & 2 & 3 & 4 & 5 \\
\hline
\end{tabular}




\section{Geo-Coordinated Parallel Coordinates (GCPC)}

These statements are related to your use of the GCPC system as a whole.

\begin{tabular}{|l|c|c|c|c|c|}
\hline General exploration of the data & $\begin{array}{c}\text { Strongly } \\
\text { Disagree }\end{array}$ & Disagree & Neutral & $\begin{array}{c}\text { Agree } \\
\begin{array}{c}\text { Strongly } \\
\text { Agree }\end{array}\end{array}$ \\
\hline $\begin{array}{l}\text { I was able to explore different subsets of the high } \\
\text { dimensional geotemporal data using the GCPC. }\end{array}$ & 1 & 2 & 3 & 4 & 5 \\
\hline $\begin{array}{l}\text { The GCPC helped me to understand the high dimensional } \\
\text { geotemporal data. }\end{array}$ & 1 & 2 & 3 & 4 & 5 \\
\hline $\begin{array}{l}\text { The GCPC helped me to explore the high dimensional } \\
\text { geotemporal data. }\end{array}$ & 1 & 2 & 3 & 4 & 5 \\
\hline $\begin{array}{l}\text { The GCPC helped me to detect patterns and trends in the } \\
\text { high dimensional geotemporal data. }\end{array}$ & 1 & 2 & 3 & 4 & 5 \\
\hline $\begin{array}{l}\text { Using the GCPC allowed me to find relevant information in } \\
\text { the high dimensional geotemporal data. }\end{array}$ & 1 & 2 & 3 & 4 & 5 \\
\hline
\end{tabular}

\begin{tabular}{|c|c|c|c|c|c|}
\hline Detailed analysis of the data & $\begin{array}{l}\text { Strongly } \\
\text { Disagree }\end{array}$ & Disagree & Neutral & Agree & $\begin{array}{l}\text { Strongly } \\
\text { Agree }\end{array}$ \\
\hline $\begin{array}{l}\text { I was able to understand relations between dimensions in } \\
\text { the data using the GCPC. }\end{array}$ & 1 & 2 & 3 & 4 & 5 \\
\hline $\begin{array}{l}\text { I was able to understand the distribution of the data using } \\
\text { the GCPC. }\end{array}$ & 1 & 2 & 3 & 4 & 5 \\
\hline $\begin{array}{l}\text { I was able to identify information that I already knew using } \\
\text { the GCPC. }\end{array}$ & 1 & 2 & 3 & 4 & 5 \\
\hline $\begin{array}{l}\text { I was able to locate new information that I didn't previously } \\
\text { know using the GCPC. }\end{array}$ & 1 & 2 & 3 & 4 & 5 \\
\hline $\begin{array}{l}\text { I was able to distinguish between different data points and } \\
\text { objects in the data set using the GCPC. }\end{array}$ & 1 & 2 & 3 & 4 & 5 \\
\hline $\begin{array}{l}\text { I was able to compare different parameters (dimensions) } \\
\text { using the GCPC. }\end{array}$ & 1 & 2 & 3 & 4 & 5 \\
\hline I was able to compare different data points using the GCPC. & 1 & 2 & 3 & 4 & 5 \\
\hline $\begin{array}{l}\text { I was able to identify relations between multi-dimensional data } \\
\text { and their geo-locations using the GCPC. }\end{array}$ & 1 & 2 & 3 & 4 & 5 \\
\hline $\begin{array}{l}\text { I was able to understand the correlations between different } \\
\text { parameters using the GCPC. }\end{array}$ & 1 & 2 & 3 & 4 & 5 \\
\hline
\end{tabular}

\begin{tabular}{|l|c|c|c|c|c|}
\hline Job relevance & $\begin{array}{c}\text { Strongly } \\
\text { Disagree }\end{array}$ & Disagree & Neutral & Agree & $\begin{array}{c}\text { Strongly } \\
\text { Agree }\end{array}$ \\
\hline $\begin{array}{l}\text { I found the GCPC relevant when analyzing high dimensional } \\
\text { geotemporal data. }\end{array}$ & 1 & 2 & 3 & 4 & 5 \\
\hline $\begin{array}{l}\text { I found the GCPC important when analyzing high } \\
\text { dimensional geotemporal data. }\end{array}$ & 1 & 2 & 3 & 4 & 5 \\
\hline
\end{tabular}




\begin{tabular}{|l|c|c|c|c|c|}
\hline Perceived ease of use & $\begin{array}{c}\text { Strongly } \\
\text { Disagree }\end{array}$ & Disagree & Neutral & Agree & $\begin{array}{c}\text { Strongly } \\
\text { Agree }\end{array}$ \\
\hline My interaction with the GCPC is clear and understandable. & 1 & 2 & 3 & 4 & 5 \\
\hline I find the GCPC easy to use. & 1 & 2 & 3 & 4 & 5 \\
\hline It was easy for me to become skilful at using the GCPC. & 1 & 2 & 3 & 4 & 5 \\
\hline I found it easy to get the GCPC to do what I wanted it to do. & 1 & 2 & 3 & 4 & 5 \\
\hline Learning to operate the GCPC was easy for me. & 1 & 2 & 3 & 4 & 5 \\
\hline I found the GCPC to be flexible to interact with. & 1 & 2 & 3 & 4 & 5 \\
\hline
\end{tabular}

\begin{tabular}{|c|c|c|c|c|c|}
\hline Perceived usefulness & $\begin{array}{l}\text { Strongly } \\
\text { Disagree }\end{array}$ & Disagree & Neutral & Agree & $\begin{array}{l}\text { Strongly } \\
\text { Agree }\end{array}$ \\
\hline $\begin{array}{l}\text { Using the GCPC improves my performance in analyzing high } \\
\text { dimensional geotemporal data. }\end{array}$ & 1 & 2 & 3 & 4 & 5 \\
\hline $\begin{array}{l}\text { Using the GCPC to analyze high dimensional geotemporal data } \\
\text { increased my productivity. }\end{array}$ & 1 & 2 & 3 & 4 & 5 \\
\hline $\begin{array}{l}\text { Using the GCPC enhances my effectiveness in analyzing high } \\
\text { dimensional geotemporal data. }\end{array}$ & 1 & 2 & 3 & 4 & 5 \\
\hline $\begin{array}{l}\text { I find the GCPC useful in analyzing high dimensional } \\
\text { geotemporal data. }\end{array}$ & 1 & 2 & 3 & 4 & 5 \\
\hline $\begin{array}{l}\text { Using the GCPC made it easier for me to analyze high } \\
\text { dimensional geotemporal data. }\end{array}$ & 1 & 2 & 3 & 4 & 5 \\
\hline $\begin{array}{l}\text { Using the GCPC in my analysis of high dimensional } \\
\text { geotemporal data enabled me to accomplish my tasks more } \\
\text { quickly. }\end{array}$ & 1 & 2 & 3 & 4 & 5 \\
\hline
\end{tabular}

\begin{tabular}{|l|c|c|c|c|c|}
\hline Output quality & $\begin{array}{c}\text { Strongly } \\
\text { Disagree }\end{array}$ & Disagree & Neutral & Agree & $\begin{array}{c}\text { Strongly } \\
\text { Agree }\end{array}$ \\
\hline The quality of the output I get from the GCPC is high. & 1 & 2 & 3 & 4 & 5 \\
\hline I have no problem with the quality of the GCPC output. & 1 & 2 & 3 & 4 & 5 \\
\hline $\begin{array}{l}\text { I have no difficulty telling others about the result of using the } \\
\text { GCPC. }\end{array}$ & 1 & 2 & 3 & 4 & 5 \\
\hline $\begin{array}{l}\text { I believe I could communicate to others the consequences of } \\
\text { using the GCPC. }\end{array}$ & 1 & 2 & 3 & 4 & 5 \\
\hline The results of using the GCPC are apparent to me. & 1 & 2 & 3 & 4 & 5 \\
\hline $\begin{array}{l}\text { I would have difficulty explaining why using the GCPC may or } \\
\text { may not be beneficial. }\end{array}$ & 1 & 2 & 3 & 4 & 5 \\
\hline
\end{tabular}




\section{Interview Questions:}

1. How long have you been conducting research with high-dimensional heterogeneous data? How long have you been analyzing environmental data?

2. How important do you think data analysis tools are to understanding environmental data?

3. How valuable do you think GCPC would be in analyzing environmental data?

4. Can you show me some examples of specific features of the data that you found using GCPC? Which of these did you know in advance? Which are new insights?

5. Please explain how you think you might be able to integrate the use of GCPC into your existing research methods?

6. Which features do you think were not particularly helpful for your tasks?

7. In your opinion, what are the features that are missing? What features do you like to see in the system?

8. How do you think you could use GCPC for explaining the result of your analysis to a colleague or other interested parties? 